\title{
NECESSARY AND SUFFICIENT LYAPUNOV-LIKE CONDITIONS FOR ROBUST NONLINEAR STABILIZATION
}

\author{
IASSON KARAFYLLIS ${ }^{1}$ AND ZHONG-PING JIANG ${ }^{2}$
}

\begin{abstract}
In this work, we propose a methodology for the expression of necessary and sufficient Lyapunov-like conditions for the existence of stabilizing feedback laws. The methodology is an extension of the well-known Control Lyapunov Function (CLF) method and can be applied to very general nonlinear time-varying systems with disturbance and control inputs, including both finite and infinitedimensional systems. The generality of the proposed methodology is also reflected upon by the fact that partial stability with respect to output variables is addressed. In addition, it is shown that the generalized CLF method can lead to a novel tool for the explicit design of robust nonlinear controllers for a class of time-delay nonlinear systems with a triangular structure.
\end{abstract}

Mathematics Subject Classification. 93D15, 93D30.

Received October 16, 2008.

Published online August 11, 2009.

\section{IntRoduction}

Feedback stabilization of nonlinear systems is a fundamentally important problem in control theory and practice. The purpose of this paper is to look at this problem from a Control Lyapunov Function point of view, but for a wide class of nonlinear time-varying systems. We aim to develop a methodology that not only results in necessary and sufficient conditions for robust feedback stabilization, but provides novel tools for the design of robust nonlinear controllers. To add to the generality of this framework, we will address partial stability with respect to output variables, instead of state variables. We first consider finite-dimensional nonlinear systems, and then show that the same methodology can be adapted to infinite-dimensional systems described by retarded functional differential equations.

Specifically, we begin with finite-dimensional control systems in the general form:

$$
\begin{aligned}
& \dot{x}(t)=f(t, d(t), x(t), u(t)) \quad Y(t)=H(t, x(t)) \\
& x(t) \in \Re^{n}, d(t) \in D, t \geqslant 0, Y(t) \in \Re^{k}, u(t) \in U \subseteq \Re^{m}
\end{aligned}
$$

where the vector fields $f: \Re^{+} \times D \times \Re^{n} \times U \rightarrow \Re^{n}, H: \Re^{+} \times \Re^{n} \rightarrow \Re^{k}$ are continuous with $f(t, d, 0,0)=0$, $H(t, 0)=0$ for all $(t, d) \in \Re^{+} \times D$. We ask the following question of feedback stabilizability: Under what

\footnotetext{
Keywords and phrases. Control Lyapunov Function, stabilization, time-varying systems, nonlinear control.

1 Department of Environmental Engineering, Technical University of Crete, 73100 Chania, Greece. ikarafyl@enveng.tuc.gr

2 Department of Electrical and Computer Engineering, Polytechnic Institute of New York University, Six Metrotech Center, Brooklyn, NY 11201, USA. zjiang@control.poly.edu
} 
conditions there exists a continuous feedback of the form:

$$
u=k(t, x)
$$

such that the closed-loop system (1.1) with (1.2) is (uniformly) Robustly Globally Asymptotically Output Stable? See Section 2.1 for a precise definition.

The above-mentioned problem has been studied by several authors in past literature for nonlinear control systems (1.1). There are two main methodologies for obtaining necessary and sufficient Lyapunov-like conditions for the existence of stabilizing feedbacks:

\section{1st Approach: The "Artstein-Sontag" methodology}

This methodology is based on the following idea: given a CLF, design the feedback so that the time derivative of the CLF along the solutions of the closed-loop system becomes negative definite.

Using this methodology in his pioneering work [2] Artstein studied the above existence problem for affine autonomous control systems without disturbances, $U \subseteq \Re^{m}$ being a closed convex set and output $Y$ being identically the state of the system, i.e., $H(t, x) \equiv x$ (see also [33]). He showed in [2] that the existence of a time-independent Control Lyapunov Function (CLF) satisfying the "small-control" property is a necessary and sufficient condition for the existence of a continuous stabilizing feedback. Sontag in [30] extended the results by presenting an explicit formula of the feedback stabilizer for affine autonomous control systems without disturbances, $U=\Re^{m}$ and output $Y$ being identically the state of the system. Sontag's formula was exploited recently in [12] for the uniform stabilization of time-varying systems. Freeman and Kokotovic in [7] extended the idea of the CLF in order to study affine control systems with disturbances, $U \subseteq \Re^{m}$ being a closed convex set and output $Y$ being identically the state of the system, i.e., $H(t, x) \equiv x$ : they introduced the concept of the Robust Control Lyapunov Function (RCLF). In [18] the authors showed that the "small-control" property is not needed for non-uniform in time robust global stabilization of the state $(H(t, x) \equiv x)$ of control systems affine in the control with $U=\Re^{m}$. The result was extended in [17] for the general case of output stability. In all the above works the stabilizing feedback is constructed using a partition of unity methodology or Michael's Theorem (when simple continuity of the feedback suffices).

\section{2nd Approach: The "Coron-Rosier" methodology}

This methodology is based on the following idea: given a CLF, design the feedback so that the difference of the values of the CLF evaluated along the solutions of the closed-loop system at time instances which differ by a constant quantity becomes negative. Notice that this methodology does not guarantee that the time derivative of the CLF along the solutions of the closed-loop system is negative definite. The method of proving asymptotic/exponential stability by means of Lyapunov functions with a non-negative definite derivative was used by Peuteman and Aeyels (see $[1,27,28]$ and references therein).

Using this methodology in their pioneering work [6] Coron and Rosier showed that the existence of a timeindependent CLF satisfying the "small-control" property is a sufficient condition for the existence of a continuous time-periodic stabilizing feedback for general non-affine disturbance-free autonomous nonlinear control systems with $U=\Re^{m}$ and output $Y$ being identically the state of the system (see also [5]). The result has been generalized in [19], where it was shown that the existence of a RCLF is a necessary and sufficient condition for the existence of a continuous time-varying stabilizing feedback for systems of the form (1.1) with $U \subseteq \Re^{m}$ being a positive cone (not necessarily convex).

It should be noted here that Control Lyapunov Functions have also been used for the design of discontinuous feedback laws (see for instance [4]), the design of static output feedback stabilizers (see [17,34]), as well as for the design of adaptive nonlinear controllers (see $[21,29])$.

However, so far the methodologies of Lyapunov design of stabilizing feedback laws are more frequently applied to finite-dimensional systems of the form (1.1). In order to be able to extend the applicability of the method to infinite-dimensional systems of the form $\dot{x}=f(t, d, x, u)$ where the state $x$ belongs to an infinite-dimensional normed linear space $X$, one has to deal with Control Lyapunov Functionals $V: \Re^{+} \times X \rightarrow \Re^{+}$, which present one (or many) of the following complications: 
(i) In contrast to CLF in the finite-dimensional case, usually Control Lyapunov Functionals are simply locally Lipschitz mappings of the state (and, not necessarily, continuously (Frechet) differentiable).

(ii) Even if the mapping $f$ is affine in $u$, the (appropriate) derivative of the Control Lyapunov Functional $\dot{V}(t, d, x, u)$ is not necessarily affine in $u$.

(iii) The existing feedback design methodology based on partition of unity arguments (see. e.g., [2,33]) does not work because the state space $X$ is infinite-dimensional.

(iv) The feedback design methodology based on Michael's Theorem (see, e.g., [7]) does not work either because simple continuity of the feedback does not suffice or because the hypotheses of Michael's Theorem cannot be verified.

Particularly, all of the above complications are encountered when control systems described by Retarded Functional Differential Equations (RFDEs) are studied, i.e., systems of the form

$$
\begin{aligned}
& \dot{x}(t)=f\left(t, d(t), T_{r}(t) x, u(t)\right), \quad Y(t)=H\left(t, T_{r}(t) x\right) \\
& x(t) \in \Re^{n}, Y(t) \in \mathcal{Y}, d(t) \in D, u(t) \in U
\end{aligned}
$$

where $r>0$ is a constant, $f: \Re^{+} \times D \times C^{0}\left([-r, 0] ; \Re^{n}\right) \times U \rightarrow \Re^{n}, H: \Re^{+} \times C^{0}\left([-r, 0] ; \Re^{n}\right) \rightarrow \mathcal{Y}$ satisfy $f(t, d, 0,0)=0, H(t, 0)=0$ for all $(t, d) \in \Re^{+} \times D, D \subseteq \Re^{l}$ is a non-empty compact set, $U \subseteq \Re^{m}$ is a closed convex set with $0 \in U, \mathcal{Y}$ is a normed linear space and $T_{r}(t) x=x(t+\theta) ; \theta \in[-r, 0]$. It should be emphasized that by allowing the output to take values in abstract normed linear spaces we are in a position to consider:

- outputs with no delays, e.g. $Y=h(t, x(t))$ with $\mathcal{Y}=\Re^{k}$;

- outputs with discrete or distributed delay, e.g. $Y(t)=h(t, x(t), x(t-r))$ or $Y(t)=\int_{t-r}^{t} h(t, \theta, x(\theta)) \mathrm{d} \theta$ with $\mathcal{Y}=\Re^{k}$;

- functional outputs with memory, e.g. $Y(t)=h(t, \theta, x(t+\theta)) ; \theta \in[-r, 0]$ or the identity output $Y(t)=$ $T_{r}(t) x=x(t+\theta) ; \theta \in[-r, 0]$ with $\mathcal{Y}=C^{0}\left([-r, 0] ; \Re^{k}\right)$.

The first contribution of the present work is the extension of the "Artstein-Sontag" methodology to the (nonaffine in the control) general finite-dimensional case (1.1). It is shown that Lipschitz RCLF can be allowed and that necessary and sufficient conditions are obtained. However, the definition of the RCLF is different from previous definitions. Particularly, certain additional properties have to be fulfilled and sufficient conditions for the derivation of the additional properties are also presented.

As the second contribution of the present work, we show how all complications mentioned above for infinitedimensional systems can be solved, and consequently, by using "Artstein-Sontag" methodology, we obtain Lyapunov-like necessary and sufficient conditions for systems of the form (1.3). We exploit the converse Lyapunov theorems in $[15,20]$ to obtain necessary and sufficient conditions for the uncertain case (1.3); the classical Lyapunov-Krasovskii characterizations (see [8]) cannot be used since they are applicable only to the disturbance-free case. Since the methodology that we describe in the present work allows the construction of locally Lipschitz stabilizing feedback laws, it is expected that it can be used for general infinite-dimensional control systems. More importantly, we will show that our generalized RCLF methodology is more than of existence-type result, but can yield constructive design tools for an enlarged class of nonlinear control systems. To this end, we will study in details a class of triangular time-delay nonlinear systems described by RFDEs, i.e.

$$
\begin{aligned}
& \dot{x}_{i}(t)=f_{i}\left(t, d(t), T_{r}(t) x_{1}, \ldots, T_{r}(t) x_{i}\right)+g_{i}\left(t, d(t), T_{r}(t) x_{1}, \ldots, T_{r}(t) x_{i}\right) x_{i+1}(t), \quad i=1, \ldots, n-1 \\
& \dot{x}_{n}(t)=f_{n}\left(t, d(t), T_{r}(t) x\right)+g_{n}\left(t, d(t), T_{r}(t) x\right) u(t) \\
& x(t)=\left(x_{1}(t), \ldots, x_{n}(t)\right) \in \Re^{n}, d(t) \in D, u(t) \in \Re, t \geqslant 0 .
\end{aligned}
$$

Lyapunov-based feedback design for various special cases of systems described by RFDEs was used recently in $[9-11,23,24,26,35]$, including the input-delayed case (not covered by (1.3)). More specifically, the stabilization problem for autonomous and disturbance-free systems of the form (1.4) has been studied in $[10,11,26,35]$. In the present work it is shown that the construction of a stabilizing feedback law for (1.4) proceeds in parallel 
with the construction of a State Robust Control Lyapunov Functional. Moreover, sufficient conditions for the existence and design of a stabilizing feedback law $u(t)=k(x(t))$, which is independent of the delay are given.

The rest of the paper is organized as follows. First, our results are developed for the finite-dimensional case (1.1) (Sect. 2), where necessary and sufficient conditions for the existence of stabilizing feedback are formulated. Section 3 is devoted to the development of sufficient conditions, which guarantee that a given function is an Output Robust Control Lyapunov function for (1.1). Examples are presented for systems which are polynomial in the control; this case was recently studied in [25]. In Section 4 we show how the same methodology can be applied to the infinite-dimensional case (1.3). Section 5 is devoted to the case of triangular control systems (1.4). Finally, our concluding remarks are given in Section 6.

Notations. Throughout this paper we adopt the following notations:

- Let $A \subseteq \Re^{n}$ be a set. By $C^{0}(A ; \Omega)$, we denote the class of continuous functions on $A$, which take values in $\Omega$. By $\bar{A}$ we denote the closure of $A \subseteq \Re^{n}$ and by $\overline{c o} A$, we denote the closure of the convex hull of $A \subseteq \Re^{n}$.

- By $C^{k}(A ; \Omega)$, where $k \geqslant 1$ is an integer, we denote the class of differentiable functions on $A$ with continuous derivatives up to order $k$, which take values in $\Omega$. By $C^{\infty}(A ; \Omega)$, we denote the class of differentiable functions on $A$ having continuous derivatives of all orders, which take values in $\Omega$, i.e., $C^{\infty}(A ; \Omega)=\bigcap_{k \geqslant 1} C^{k}(A ; \Omega)$.

- By \|\|$_{\mathcal{Y}}$, we denote the norm of the normed linear space $\mathcal{Y} . \Re^{+}$denotes the set of non-negative real numbers.

- For a vector $x \in \Re^{n}$ we denote by $|x|$ its usual Euclidean norm and by $x^{\prime}$ its transpose. For $x \in$ $C^{0}\left([-r, 0] ; \Re^{n}\right)$ we define $\|x\|_{r}:=\max _{\theta \in[-r, 0]}|x(\theta)|$.

- A continuous mapping $A \times B$ э $(z, x) \rightarrow k(z, x) \in \Re^{m}$, where $B \subseteq \mathcal{X}, A \subseteq \mathcal{Y}$ and $\mathcal{X}, \mathcal{Y}$ are normed linear spaces, is called completely locally Lipschitz with respect to $x \in B$ if for every closed and bounded set $S \subseteq A \times B$ it holds that $\sup \left\{\frac{|k(z, x)-k(z, y)|}{\|x-y\|_{\mathcal{X}}}:(z, x) \in S,(z, y) \in S, x \neq y\right\}<+\infty$. If the normed linear spaces $\mathcal{X}, \mathcal{Y}$ are finite-dimensional spaces then we simply say that the continuous mapping $A \times B$ э $(z, x) \rightarrow k(z, x) \in \Re^{m}$ is locally Lipschitz with respect to $x \in B$ if for every compact set $S \subseteq A \times B$ it holds that $\sup \left\{\frac{|k(z, x)-k(z, y)|}{|x-y|}:(z, x) \in S,(z, y) \in S, x \neq y\right\}<+\infty$.

- We denote by $K^{+}$the class of positive $C^{0}$ functions defined on $\Re^{+}$. We say that a function $\rho: \Re^{+} \rightarrow \Re^{+}$ is positive definite if $\rho(0)=0$ and $\rho(s)>0$ for all $s>0$. By $K$ we denote the set of positive definite, increasing and continuous functions. We say that a positive definite, increasing and continuous function $\rho: \Re^{+} \rightarrow \Re^{+}$is of class $K_{\infty}$ if $\lim _{s \rightarrow+\infty} \rho(s)=+\infty$. By $K L$ we denote the set of all continuous functions $\sigma=\sigma(s, t): \Re^{+} \times \Re^{+} \rightarrow \Re^{+}$with the properties: (i) for each $t \geqslant 0$ the mapping $\sigma(\cdot, t)$ is of class $K$; (ii) for each $s \geqslant 0$, the mapping $\sigma(s, \cdot)$ is non-increasing with $\lim _{t \rightarrow+\infty} \sigma(s, t)=0$. $\boldsymbol{E}$ denotes the class of non-negative $C^{0}$ functions $\mu: \Re^{+} \rightarrow \Re^{+}$, for which it holds: $\int_{0}^{+\infty} \mu(t) \mathrm{d} t<+\infty$ and $\lim _{t \rightarrow+\infty} \mu(t)=0$.

- Let $D \subseteq \Re^{l}$ be a non-empty set. By $M_{D}$ we denote the class of all Lebesgue measurable and locally essentially bounded mappings $d: \Re^{+} \rightarrow D$.

- Let $x:[a-r, b) \rightarrow \Re^{n}$ be a continuous mapping with $b>a>-\infty$ and $r>0$. By $T_{r}(t) x$ we denote the "r-history" of $x$ at time $t \in[a, b)$, i.e., $T_{r}(t) x:=x(t+\theta) ; \theta \in[-r, 0]$.

- A function $\Psi: A \times U \rightarrow \Re \cup\{+\infty\}$, where $A \subseteq \Re^{n}, U \subseteq \Re^{m}$ is a convex set, is called quasi-convex with respect to $u \in U$, if for every $u, v \in U, x \in A$ and $\lambda \in[0,1]$ it holds that $\Psi(x, \lambda u+(1-\lambda) v) \leqslant$ $\max \{\Psi(x, u), \Psi(x, v)\}$.

- Let $U \subseteq \Re^{n}$ be a non-empty closed set. $\operatorname{By} \operatorname{Pr}_{U}(x)$, we denote the projection of $x \in \Re^{n}$ on $U \subseteq \Re^{n}$. Notice that if $U \subseteq \Re^{n}$ is convex then $\left|\operatorname{Pr}_{U}(x)-\operatorname{Pr}_{U}(y)\right| \leqslant|x-y|$, for all $x, y \in \Re^{n}$. 


\section{Finite-DimEnSIONAL CONTROL SYSTEMS}

In this section, we consider control systems of the form (1.1) under the following hypotheses:

(H1) The vector fields $f: \Re^{+} \times D \times \Re^{n} \times U \rightarrow \Re^{n}, H: \Re^{+} \times \Re^{n} \rightarrow \Re^{k}$ are continuous and for every bounded interval $I \subset \Re^{+}$and every compact set $S \subset \Re^{n} \times U$ there exists $L \geqslant 0$ such that $|f(t, d, x, u)-f(t, d, y, v)| \leqslant$ $L|x-y|+L|u-v|$ for all $(t, d) \in I \times D,(x, u) \in S,(y, v) \in S\left(i . e\right.$., the mapping $\Re^{+} \times D \times \Re^{n} \times U$ э $(t, d, x, u) \rightarrow$ $f(t, d, x, u) \in \Re^{n}$ is locally Lipschitz with respect to $\left.(x, u)\right)$.

(H2) The set $D \subset \Re^{l}$ is compact and $U \subseteq \Re^{m}$ is a closed convex set.

(H3) $f(t, d, 0,0)=0, H(t, 0)=0$ for all $(t, d) \in \Re^{+} \times D$.

In order to present the main results on finite-dimensional systems of the form (1.1) we need to present in detail the basic steps of the method. The methodology consists of the following steps:

2.1. Notions of output stability

2.2. Lyapunov-like criteria for output stability

2.3. Definition of the output robust control Lyapunov function

2.4. Converse Lyapunov theorems for output stability.

\subsection{Notions of output stability}

We first analyze the output stability notions used in the present work. Consider the system

$$
\begin{aligned}
& \dot{x}(t)=f(t, d(t), x(t)) \quad Y(t)=H(t, x(t)) \\
& x(t) \in \Re^{n}, d(t) \in D, Y(t) \in \Re^{k}
\end{aligned}
$$

where the vector fields $f: \Re^{+} \times D \times \Re^{n} \rightarrow \Re^{n}, H: \Re^{+} \times \Re^{n} \rightarrow \Re^{k}$ are continuous and $D \subset \Re^{l}$ is compact. We assume that for every $\left(t_{0}, x_{0}, d\right) \in \Re^{+} \times \Re^{n} \times M_{D}$ there exist $h \in(0,+\infty]$ and a unique absolutely continuous mapping $x:\left[t_{0}, t_{0}+h\right) \rightarrow \Re^{n}$ with $x\left(t_{0}\right)=x_{0}$ and $\dot{x}(t)=f(t, d(t), x(t))$ a.e. for $t \in\left[t_{0}, t_{0}+h\right)$. Moreover, we assume that $f(t, d, 0)=0, H(t, 0)=0$ for all $(t, d) \in \Re^{+} \times D$. The solution $x:\left[t_{0}, t_{0}+h\right) \rightarrow \Re^{n}$ of $(2.1)$ at time $t \geqslant t_{0}$ with initial condition $x\left(t_{0}\right)=x_{0}$ corresponding to input $d \in M_{D}$ will be denoted by $x\left(t, t_{0}, x_{0} ; d\right)$.

Definition 2.1. We say that (2.1) is Robustly Forward Complete $(R F C)$ if for every $T \geqslant 0, r \geqslant 0$ it holds that:

$$
\sup \left\{\left|x\left(t_{0}+h, t_{0}, x_{0} ; d\right)\right| ;\left|x_{0}\right| \leqslant r, t_{0} \in[0, T], h \in[0, T], d(\cdot) \in M_{D}\right\}<+\infty .
$$

Clearly, the notion of robust forward completeness implies the standard notion of forward completeness, which simply requires that for every initial condition the solution of the system exists for all times greater than the initial time, or equivalently, the solutions of the system do not present finite escape time. Conversely, an extension of Proposition 5.1 in [22] to the time-varying case shows that every forward complete system (2.1) whose dynamics are locally Lipschitz with respect to $(t, x)$, uniformly in $d \in D$, is RFC. All output stability notions used in the present work will assume RFC.

We continue with the notion of (non-uniform in time) Robust Global Asymptotic Output Stability (RGAOS) as a generalization of the notion of Robust Output Stability (see $[13,14]$ ). Let us denote by $Y(t)=$ $H\left(t, x\left(t, t_{0}, x_{0} ; d\right)\right)$ the value of the output for the unique solution of (2.1) at time $t$ that corresponds to input $d \in M_{D}$ with initial condition $x\left(t_{0}\right)=x_{0}$.

Definition 2.2. Consider system (2.1) and suppose that (2.1) is RFC. We say that system (2.1) is (nonuniformly in time) Robustly Globally Asymptotically Output Stable (RGAOS) if it satisfies the following properties:

P1 (Output Stability). For every $\varepsilon>0, T \geqslant 0$, it holds that

$$
\sup \left\{|Y(t)| ; t \geqslant t_{0},\left|x_{0}\right| \leqslant \varepsilon, t_{0} \in[0, T], d(\cdot) \in M_{D}\right\}<+\infty
$$


and there exists a $\delta:=\delta(\varepsilon, T)>0$ such that:

$$
\left|x_{0}\right| \leqslant \delta, t_{0} \in[0, T] \Rightarrow|Y(t)| \leqslant \varepsilon, \quad \forall t \geqslant t_{0}, \forall d(\cdot) \in M_{D} .
$$

P2 (Uniform Output Attractivity on compact sets of initial data). For every $\varepsilon>0, T \geqslant 0$ and $R \geqslant 0$, there exists a $\tau:=\tau(\varepsilon, T, R) \geqslant 0$, such that:

$$
\left|x_{0}\right| \leqslant R, t_{0} \in[0, T] \Rightarrow|Y(t)| \leqslant \varepsilon, \quad \forall t \geqslant t_{0}+\tau, \forall d(\cdot) \in M_{D} .
$$

The notion of Uniform Robust Global Asymptotic Output Stability was given in [31,32] and is a special case of (non-uniform in time) RGAOS.

Definition 2.3. Consider system (2.1) and suppose that (2.1) is RFC. We say that system (2.1) is Uniformly Robustly Globally Asymptotically Output Stable (URGAOS) if it satisfies the following properties:

P1 (Uniform Output Stability). For every $\varepsilon>0$, it holds that

$$
\sup \left\{|Y(t)| ; t \geqslant t_{0},\left|x_{0}\right| \leqslant \varepsilon, t_{0} \geqslant 0, d(\cdot) \in M_{D}\right\}<+\infty
$$

and there exists a $\delta:=\delta(\varepsilon)>0$ such that:

$$
\left|x_{0}\right| \leqslant \delta, t_{0} \geqslant 0 \Rightarrow|Y(t)| \leqslant \varepsilon, \quad \forall t \geqslant t_{0}, \forall d(\cdot) \in M_{D} .
$$

P2 (Uniform Output Attractivity on compact sets of initial states). For every $\varepsilon>0$ and $R \geqslant 0$, there exists a $\tau:=\tau(\varepsilon, R) \geqslant 0$, such that:

$$
\left|x_{0}\right| \leqslant R, t_{0} \geqslant 0 \Rightarrow|Y(t)| \leqslant \varepsilon, \quad \forall t \geqslant t_{0}+\tau, \forall d(\cdot) \in M_{D} .
$$

Obviously, for the case $H(t, x)=x$ the notions of RGAOS and URGAOS coincide with the notions of non-uniform in time Robust Global Asymptotic Stability (RGAS) as given in [18] and Uniform Robust Global Asymptotic Stability (URGAS) as given in [22], respectively. Also note that if there exists $a \in K_{\infty}$ with $|x| \leqslant a(|H(t, x)|)$ for all $(t, x) \in \Re^{+} \times \Re^{n}$, then (U)RGAOS implies (U)RGAS.

\subsection{Lyapunov-like criteria for output stability}

For a locally bounded function $V: \Re^{+} \times \Re^{n} \rightarrow \Re$, we define

$$
V^{0}(t, x ; v):=\limsup _{h \rightarrow 0^{+}, w \rightarrow v} \frac{V(t+h, x+h w)-V(t, x)}{h} .
$$

The reader should notice that the function $(t, x, v) \rightarrow V^{0}(t, x ; v)$ may take values in the extended real number set $\Re^{*}=[-\infty,+\infty]$. However, for locally Lipschitz functions $V: \Re^{+} \times \Re^{n} \rightarrow \Re$, the function $(t, x, v) \rightarrow V^{0}(t, x ; v)$ is locally bounded. It should be clear that for locally Lipschitz functions $V: \Re^{+} \times \Re^{n} \rightarrow \Re$ it holds that:

$$
V^{0}(t, x ; v)=\limsup _{h \rightarrow 0^{+}} \frac{V(t+h, x+h v)-V(t, x)}{h} .
$$

The main reason for introducing the above Dini derivative is the following lemma.

Lemma 2.4. Let $V: \Re^{+} \times \Re^{n} \rightarrow \Re$ be a locally bounded function and let $x:\left[t_{0}, t_{\max }\right) \rightarrow \Re^{n}$ be a solution of (2.1) with initial condition $x\left(t_{0}\right)=x_{0} \in \Re^{n}$ corresponding to certain $d \in M_{D}$, where $t_{\max } \in\left(t_{0},+\infty\right]$ is the maximal existence time of the solution. Then it holds that

$$
\limsup _{h \rightarrow 0^{+}} h^{-1}(V(t+h, x(t+h))-V(t, x(t))) \leqslant V^{0}\left(t, x ; D^{+} x(t)\right) \text {, a.e. on }\left[t_{0}, t_{\max }\right)
$$

where $D^{+} x(t)=\lim _{h \rightarrow 0^{+}} h^{-1}(x(t+h)-x(t))$. 
Proof. Inequality (2.5) follows directly from definition (2.3) and definition $w_{h}=\frac{x(t+h)-x(t)}{h}$, where $t \in\left[t_{0}, t_{\max }\right) \backslash N$ and $N$ is a measure zero set where $D^{+} x(t)=\lim _{h \rightarrow 0^{+}} h^{-1}(x(t+h)-x(t))$ is not defined. Notice that since $D^{+} x(t)=\lim _{h \rightarrow 0^{+}} h^{-1}(x(t+h)-x(t))$ we obtain that $w_{h} \rightarrow D^{+} x(t)$ as $h \rightarrow 0^{+}$. The proof is complete.

Having introduced an appropriate derivative for Lyapunov functions, we are now in a position to give Lyapunov-like criteria for RGAOS and URGAOS. The proof of the following proposition can be found in the Appendix.

Proposition 2.5. Consider system (2.1) and the following statements:

(Q1) There exist a locally Lipschitz function $V: \Re^{+} \times \Re^{n} \rightarrow \Re^{+}$, functions $a_{1}, a_{2} \in K_{\infty}, \beta, \mu \in K^{+}$, a function $q \in \mathcal{E}$ and a $C^{0}$ positive definite function $\rho: \Re^{+} \rightarrow \Re^{+}$such that

$$
a_{1}(|(\mu(t) x, H(t, x))|) \leqslant V(t, x) \leqslant a_{2}(\beta(t)|x|), \quad \forall(t, x) \in \Re^{+} \times \Re^{n}
$$

and such that the following inequality holds for all $(t, x, d) \in \Re^{+} \times \Re^{n} \times D$ :

$$
V^{0}(t, x ; f(t, d, x)) \leqslant-\rho(V(t, x))+q(t) .
$$

(Q2) Hypothesis (Q1) holds with $\beta(t) \equiv 1, q(t) \equiv 0$.

(Q3) The mapping $\Re^{+} \times D \times \Re^{n} \ni(t, d, x) \rightarrow f(t, d, x) \in \Re^{n}$ is locally Lipschitz with respect to $x \in \Re^{n}$.

If hypotheses (Q1), (Q3) hold then system (2.1) is RGAOS. If hypothesis (Q2) holds, then system (2.1) is URGAOS.

\subsection{Definition of the output robust control Lyapunov function}

We next give the definition of the Output Robust Control Lyapunov Function for system (1.1). The definition is in the same spirit with the definition of the notion of Robust Control Lyapunov Function given in [7] for continuous-time finite-dimensional control systems. The small-control property in the following definition constitutes a time-varying version of the small-control property for the autonomous case $[2,7,30]$.

Definition 2.6. We say that (1.1) admits an Output Robust Control Lyapunov Function (ORCLF) if there exists a locally Lipschitz function $V: \Re^{+} \times \Re^{n} \rightarrow \Re^{+}$(called the Output Robust Control Lyapunov Function), which satisfies the following properties:

(i) There exist $a_{1}, a_{2} \in K_{\infty}, \beta, \mu \in K^{+}$such that (2.6) holds.

(ii) There exists a function $\Psi: \Re^{+} \times \Re^{n} \times U \rightarrow \Re \cup\{+\infty\}$ with $\Psi(t, 0,0)=0$ for all $t \geqslant 0$ such that for each $u \in U$ the mapping $(t, x) \rightarrow \Psi(t, x, u)$ is upper semi-continuous, a function $q \in \mathcal{E}$ and a $C^{0}$ positive definite function $\rho: \Re^{+} \rightarrow \Re^{+}$such that the following inequality holds:

$$
\inf _{u \in U} \Psi(t, x, u) \leqslant q(t), \quad \forall(t, x) \in \Re^{+} \times \Re^{n} .
$$

Moreover, for every finite set $\left\{u_{1}, u_{2}, \ldots, u_{p}\right\} \subset U$ and for every $\lambda_{i} \in[0,1](i=1, \ldots, p)$ with $\sum_{i=1}^{p} \lambda_{i}=1$, it holds that:

$$
\sup _{d \in D} V^{0}\left(t, x ; f\left(t, d, x, \sum_{i=1}^{p} \lambda_{i} u_{i}\right)\right) \leqslant-\rho(V(t, x))+\max \left\{\Psi\left(t, x, u_{i}\right), i=1, \ldots, p\right\}, \quad \forall(t, x) \in \Re^{+} \times \Re^{n} .
$$


If in addition to the above there exist $a \in K_{\infty}, \gamma \in K^{+}$such that for every $(t, x) \in \Re^{+} \times \Re^{n}$ there exists $u \in U$ with $|u| \leqslant a(\gamma(t)|x|)$ such that

$$
\Psi(t, x, u) \leqslant q(t)
$$

then we say that $V: \Re^{+} \times \Re^{n} \rightarrow \Re^{+}$satisfies the "small-control" property.

For the case $H(t, x) \equiv x$ we simply call $V: \Re^{+} \times \Re^{n} \rightarrow \Re^{+}$a State Robust Control Lyapunov Function (SRCLF).

Remark 2.7. It is important to emphasize that the Dini derivative used for the Lyapunov-like criteria for output stability (Prop. 2.5) is the same derivative used in inequality (2.9) for the definition of the ORCLF.

\subsection{Converse Lyapunov theorems for output stability}

In the rest of Section 2, we are going to exploit the converse Lyapunov theorem for RGAOS presented in [14].

\subsection{Main results}

We are now ready to state and prove our main results for the finite-dimensional case (1.1).

Theorem 2.8. Consider system (1.1) under hypotheses (H1)-(H3). The following statements are equivalent:

(a) There exists a $C^{\infty}$ function $k: \Re^{+} \times \Re^{n} \rightarrow U$ with $k(t, 0)=0$ for all $t \geqslant 0$, in such a way that the closed-loop system (1.1) with $u=k(t, x)$ is RGAOS.

(b) There exists a $C^{0}$ function $k: \Re^{+} \times \Re^{n} \rightarrow U$ with $f(t, d, x, k(t, x))$ being locally Lipschitz with respect to $x$ and $f(t, d, 0, k(t, 0))=0$ for all $(t, d) \in \Re^{+} \times D$, such that the closed-loop system (1.1) with $u=k(t, x)$ is $R G A O S$.

(c) System (1.1) admits an ORCLF, which satisfies the small control property with $q(t) \equiv 0$.

(d) System (1.1) admits an ORCLF.

Theorem 2.9. Consider system (1.1) under hypotheses (H1)-(H3). If system (1.1) admits an ORCLF, which satisfies the small-control property and inequalities $(2.6),(2.10)$ with $\beta(t) \equiv 1, q(t) \equiv 0$, then there exists a continuous mapping $k: \Re^{+} \times \Re^{n} \rightarrow U$, with $k(t, 0)=0$ for all $t \geqslant 0$, which is $C^{\infty}$ on the set $\Re^{+} \times\left(\Re^{n} \backslash\{0\}\right)$, such that

(i) for all $\left(t_{0}, x_{0}, d\right) \in \Re^{+} \times \Re^{n} \times M_{D}$ the solution $x(t)$ of the closed-loop system (1.1) with $u=k(t, x)$, i.e. the solution of

$$
\dot{x}(t)=f(t, d(t), x(t), k(t, x(t)))
$$

with initial condition $x\left(t_{0}\right)=x_{0} \in \Re^{n}$, corresponding to input $d \in M_{D}$ is unique,

(ii) system (2.11) is URGAOS.

Moreover, if the ORCLF $V$ and the function $\Psi$ involved in property (ii) of Definition 2.6 are time independent then the continuous mapping $k$ is time invariant. Finally, if in addition there exist functions $\eta \in K^{+}, \varphi \in$ $C^{v}(A ; U)$ where $v \in\{1,2, \ldots\}, A=\cup_{t \geqslant 0}\{t\} \times\left\{x \in \Re^{n}:|x|<4 \eta(t)\right\}$ with $\varphi(t, 0)=0$ for all $t \geqslant 0$, such that

$$
\Psi(t, x, \varphi(t, x)) \leqslant 0, \text { for all }(t, x) \in \Re^{+} \times \Re^{n} \text { with }|x| \leqslant 2 \eta(t)
$$

then the continuous mapping $k$ is of class $C^{v}\left(\Re^{+} \times \Re^{n} ; U\right)$.

Proof of Theorem 2.8. The implications $(\mathrm{a}) \Rightarrow(\mathrm{b})$ and $(\mathrm{c}) \Rightarrow(\mathrm{d})$ are obvious and we prove implications $(\mathrm{d}) \Rightarrow(\mathrm{a})$, $(\mathrm{b}) \Rightarrow(\mathrm{d})$ and $(\mathrm{a}) \Rightarrow(\mathrm{c})$.

$(\mathrm{d}) \Rightarrow(\mathbf{a})$. Suppose that (1.1) admits an ORCLF. Without loss of generality, we may assume that the function $q \in \boldsymbol{E}$ involved in (2.8) is positive for all $t \geqslant 0$. 
Furthermore define:

$$
\begin{gathered}
\Xi(t, x, u):=\Psi(t, x, u)-8 q(t), \quad(t, x, u) \in \Re^{+} \times \Re^{n} \times U \\
\Xi(t, x, u):=\Xi(0, x, u), \quad(t, x, u) \in(-1,0) \times \Re^{n} \times U .
\end{gathered}
$$

The definition of $\Xi$, given by (2.13a), (2.13b), guarantees that the function $\Xi:(-1,+\infty) \times \Re \Re^{n} \times U \rightarrow \Re \cup\{+\infty\}$ with $\Xi(t, 0,0)=-8 q(\max \{0, t\})$ for all $t>-1$ is such that, for each $u \in U$ the mapping $(t, x) \rightarrow \Xi(t, x, u)$ is upper semi-continuous. By virtue of (2.8) and upper semi-continuity of $\Xi$, it follows that for each $(t, x) \in$ $(-1,+\infty) \times \Re^{n}$ there exist $u=u(t, x) \in U$ and $\delta=\delta(t, x) \in(0, t+1)$, such that

$$
\Xi(\tau, y, u(t, x)) \leqslant 0, \forall(\tau, y) \in\left\{(\tau, y) \in(-1,+\infty) \times \Re^{n}:|\tau-t|+|y-x|<\delta\right\}
$$

Using (2.14) and standard partition of unity arguments, we can determine sequences $\left\{\left(t_{i}, x_{i}\right) \in(-1,+\infty) \times\right.$ $\left.\Re^{n}\right\}_{i=1}^{\infty},\left\{u_{i} \in U\right\}_{i=1}^{\infty},\left\{\delta_{i}\right\}_{i=1}^{\infty}$ with $\delta_{i}=\delta\left(t_{i}, x_{i}\right) \in\left(0, t_{i}+1\right)$ associated with a sequence of open sets $\left\{\Omega_{i}\right\}_{i=1}^{\infty}$ with

$$
\Omega_{i} \subseteq\left\{(\tau, y) \in(-1,+\infty) \times \Re^{n}:\left|\tau-t_{i}\right|+\left|y-x_{i}\right|<\delta_{i}\right\}
$$

forming a locally finite open covering of $(-1,+\infty) \times \Re^{n}$ and in such a way that:

$$
\Xi\left(\tau, y, u_{i}\right) \leqslant 0, \quad \forall(\tau, y) \in \Omega_{i}
$$

Also, a family of smooth functions $\left\{\theta_{i}\right\}_{i=1}^{\infty}$ with $\theta_{i}(t, x) \geqslant 0$ for all $(t, x) \in(-1,+\infty) \times \Re^{n}$ can be determined with

$$
\begin{gathered}
\operatorname{supp} \theta_{i} \subseteq \Omega_{i} \\
\sum_{i=1}^{\infty} \theta_{i}(t, x)=1, \quad \forall(t, x) \in(-1,+\infty) \times \Re^{n} .
\end{gathered}
$$

The facts that $\Xi(t, 0,0)=-8 q(t)<0$ for all $t \geqslant 0$ and that the mapping $(t, x) \rightarrow \Xi(t, x, 0)$ is upper semicontinuous, imply that for every $t \geqslant 0$ there exists $\delta(t)>0$ such that $\Xi(\tau, y, 0) \leqslant 0$ for all $(\tau, y) \in \Re^{+} \times \Re^{n}$ with $|\tau-t|+|y| \leqslant \delta(t)$. Utilizing compactness of $[0, T]$ for every $T \geqslant 0$, we conclude that for every $T \geqslant 0$ there exists $\tilde{\delta}(T)>0$ such that

$$
(\tau, y) \in[0, T] \times \Re^{n} \text { and }|y| \leqslant \tilde{\delta}(T) \quad \Rightarrow \Xi(\tau, y, 0) \leqslant 0
$$

Define the following function:

$$
\tilde{\eta}(t):=\frac{1}{2}([t]+1-t) \tilde{\delta}([t]+1)+\frac{1}{2}(t-[t]) \tilde{\delta}([t]+2), \quad t \geqslant 0
$$

where $[t]$ denotes the integer part of $t \geqslant 0$. Notice that by virtue of definition (2.20) it follows that $\tilde{\eta}(k)=$ $\frac{1}{2} \tilde{\delta}(k+1), \lim _{t \rightarrow(k+1)^{-}} \tilde{\eta}(t)=\frac{1}{2} \tilde{\delta}(k+2)$ for all $k \in Z^{+}$, which implies that $\tilde{\eta}$ is continuous. Moreover, definition (2.20) gives $0<\tilde{\eta}(t) \leqslant \frac{1}{2} \max \{\tilde{\delta}([t]+1) ; \tilde{\delta}([t]+2)\}$ for all $t \geqslant 0$, which in conjunction with (2.19) and the inequality $t \leqslant[t]+1 \leqslant[t]+2$ implies:

$$
|x| \leqslant 2 \tilde{\eta}(t) \Rightarrow \Xi(t, x, 0) \leqslant 0
$$

Let $\bar{\eta}: \Re^{+} \rightarrow(0,+\infty)$ be the positive, continuous and non-decreasing function defined by $\bar{\eta}(t):=\min _{0 \leqslant \tau \leqslant t} \tilde{\eta}(\tau)$. Let $\varphi \in C^{\infty}(\Re ;[0,1])$ be a smooth function with $\int_{0}^{1} \varphi(s) \mathrm{d} s>0, \varphi(s)=0$ for all $s \leqslant 0$ and $s \geqslant 1$. 
Define $\eta(t):=\int_{0}^{1} \varphi(s) \bar{\eta}(t+s) \mathrm{d} s$, which is a $C^{\infty}$ positive function that satisfies $\eta(t) \leqslant \tilde{\eta}(t)$ for all $t \geqslant 0$. Consequently, by virtue of (2.21) we obtain:

$$
|x| \leqslant 2 \eta(t) \Rightarrow \Xi(t, x, 0) \leqslant 0
$$

Let $h \in C^{\infty}(\Re ;[0,1])$ be a smooth non-decreasing function with $h(s)=0$ for all $s \leqslant 0$ and $h(s)=1$ for all $s \geqslant 1$. We define:

$$
k(t, x):=h\left(\frac{|x|^{2}-2 \eta^{2}(t)}{2 \eta^{2}(t)}\right) \sum_{i=1}^{\infty} \theta_{i}(t, x) u_{i}
$$

Clearly, $k$ as defined by (2.23) is a smooth function with $k(t, 0)=0$ for all $t \geqslant 0$. Moreover, since $k(t, x)$ is defined as a (finite) convex combination of $u_{i} \in U$ and $0 \in U$, we have $k(t, x) \in U$ for all $(t, x) \in \Re^{+} \times \Re^{n}$.

Let $(t, x) \in \Re^{+} \times \Re^{n}$ with $|x| \geqslant 2 \eta(t)$ and define $J(t, x)=\left\{j \in\{1,2, \ldots\} ; \theta_{j}(t, x) \neq 0\right\}$ (a finite set). Notice that by virtue of (2.9) and definition (2.23) we get:

$$
\begin{aligned}
\sup _{d \in D} V^{0}(t, x ; f(t, d, x, k(t, x))) & =\sup _{d \in D} V^{0}\left(t, x ; f\left(t, d, x, \sum_{j \in J(t, x)} \theta_{j}(t, x) u_{j}\right)\right) \\
& \leqslant-\rho(V(t, x))+\max _{j \in J(t, x)}\left\{\Psi\left(t, x, u_{j}\right)\right\} .
\end{aligned}
$$

Notice that for each $j \in J(t, x)$ we obtain from (2.17) that $(t, x) \in \Omega_{j}$. Consequently, by virtue of (2.16) and definition (2.13a) we have that $\Psi\left(t, x, u_{j}\right) \leqslant 8 q(t)$, for all $j \in J(t, x)$. Combining the previous inequality with inequality (2.24), we conclude that the following property holds for all $(t, x, d) \in \Re^{+} \times \Re^{n} \times D$ with $|x| \geqslant 2 \eta(t)$ :

$$
V^{0}(t, x ; f(t, d, x, k(t, x))) \leqslant-\rho(V(t, x))+8 q(t) .
$$

Let $(t, x) \in \Re^{+} \times \Re^{n}$ with $|x| \leqslant \sqrt{2} \eta(t)$. Notice that by virtue of definition (2.23) we get:

$$
\sup _{d \in D} V^{0}(t, x ; f(t, d, x, k(t, x)))=\sup _{d \in D} V^{0}(t, x ; f(t, d, x, 0)) .
$$

By virtue of (2.22), (2.13a) and the above inequality we conclude that (2.25) holds as well for all $(t, x) \in$ $\Re^{+} \times \Re^{n}$ with $|x| \leqslant \sqrt{2} \eta(t)$. Finally, for the case $(t, x) \in \Re^{+} \times \Re^{n}$ with $\sqrt{2} \eta(t)<|x|<2 \eta(t)$, let $J(t, x)=$ $\left\{j \in\{1,2, \ldots\} ; \theta_{j}(t, x) \neq 0\right\}$ and notice that from (2.9) we get:

$$
\begin{aligned}
\sup _{d \in D} V^{0}(t, x ; f(t, d, x, k(t, x))) & =\sup _{d \in D} V^{0}\left(t, x ; f\left(t, d, x, h\left(\frac{|x|^{2}-2 \eta^{2}(t)}{2 \eta^{2}(t)}\right) \sum_{j \in J(t, x)} \theta_{j}(t, x) u_{j}\right)\right) \\
& \leqslant-\rho(V(t, x))+\max \left\{\Psi(t, x, 0), \Psi\left(t, x, u_{j}\right), j \in J(t, x)\right\} .
\end{aligned}
$$

Taking into account definition (2.13a) and inequalities (2.16), (2.17), (2.22), (2.26) we may conclude that (2.25) holds as well for all $(t, x) \in \Re^{+} \times \Re^{n}$ with $\sqrt{2} \eta(t)<|x|<2 \eta(t)$. Consequently, (2.25) holds for all $(t, x) \in$ $\Re^{+} \times \Re^{n}$.

It follows from (2.25) and Proposition 2.5 that system (1.1) with $u=k(t, x)$ is RGAOS.

(b) $\Rightarrow(\mathbf{d})$. Since system (1.1) with $u=k(t, x)$ is RGAOS, and since $f(t, d, x, k(t, x))$ is Lipschitz with respect to $x$ on each bounded subset of $\Re^{+} \times \Re^{n} \times D$, it follows from Theorem 3.2 in [14] that there exists a function 
$V \in C^{\infty}\left(\Re^{+} \times \Re^{n} ; \Re^{+}\right)$, functions $a_{1}, a_{2} \in K_{\infty}, \beta, \mu \in K^{+}$such that

$$
\begin{gathered}
a_{1}(|(\mu(t) x, H(t, x))|) \leqslant V(t, x) \leqslant a_{2}(\beta(t)|x|), \quad \forall(t, x) \in \Re^{+} \times \Re^{n} \\
\frac{\partial V}{\partial t}(t, x)+\sup _{d \in D}\left(\frac{\partial V}{\partial x}(t, x) f(t, d, x, k(t, x))\right) \leqslant-V(t, x), \quad \forall(t, x) \in \Re^{+} \times \Re^{n} .
\end{gathered}
$$

We next prove that $V$ is an ORCLF for (1.1). Obviously property (i) of Definition 2.6 is a consequence of inequality (2.27a). Define

$$
\Psi(t, x, u):=V(t, x)+\sup \left\{\frac{\partial V}{\partial t}(t, x)+\frac{\partial V}{\partial x}(t, x) f(t, d, x, v): d \in D, v \in U \text { with }|v-k(t, x)| \leqslant|u-k(t, x)|\right\} .
$$

Inequality (2.8) with $q(t) \equiv 0$ is an immediate consequence of inequality (2.27b) and definition (2.28). Moreover, it holds that

$$
\frac{\partial V}{\partial t}(t, x)+\sup _{d \in D}\left(\frac{\partial V}{\partial x}(t, x) f(t, d, x, u)\right) \leqslant-V(t, x)+\Psi(t, x, u), \quad \forall(t, x, u) \in \Re^{+} \times \Re^{n} \times U .
$$

It follows from compactness of $D \subset \Re^{l}$, continuity of $V(t, x), \frac{\partial V}{\partial t}(t, x)+\frac{\partial V}{\partial x}(t, x) f(t, d, x, v), k: \Re^{+} \times \Re^{n} \rightarrow U$ and Theorem 1.4.16 in [3] that the function $\Psi$ as defined by (2.28) is upper semi-continuous. Clearly, definition (2.28) implies $\Psi(t, 0,0)=0$ for all $t \geqslant 0$.

Finally, we show that inequality $(2.9)$ holds with $\rho(s):=s$. Let arbitrary $(t, x) \in \Re^{+} \times \Re^{n},\left\{u_{1}, u_{2}, \ldots, u_{p}\right\} \subset U$ and $\lambda_{i} \in[0,1](i=1, \ldots, p)$ with $\sum_{i=1}^{p} \lambda_{i}=1$. Definition (2.28) implies:

$$
\begin{aligned}
& \Psi\left(t, x, \sum_{i=1}^{p} \lambda_{i} u_{i}\right)=V(t, x)+\sup \left\{\frac{\partial V}{\partial t}(t, x)+\frac{\partial V}{\partial x}(t, x) f(t, d, x, v): d \in D, v \in U\right. \\
& \text { with } \left.|v-k(t, x)| \leqslant\left|\sum_{i=1}^{p} \lambda_{i} u_{i}-k(t, x)\right|\right\} \\
& \leqslant V(t, x)+\sup \left\{\frac{\partial V}{\partial t}(t, x)+\frac{\partial V}{\partial x}(t, x) f(t, d, x, v): d \in D, v \in U\right. \\
& \text { with } \left.|v-k(t, x)| \leqslant \sum_{i=1}^{p} \lambda_{i}\left|u_{i}-k(t, x)\right|\right\} \\
& \leqslant V(t, x)+\sup \left\{\frac{\partial V}{\partial t}(t, x)+\frac{\partial V}{\partial x}(t, x) f(t, d, x, v): d \in D, v \in U\right. \\
& \text { with } \left.|v-k(t, x)| \leqslant \max _{i=1, \ldots, p}\left|u_{i}-k(t, x)\right|\right\} \text {. }
\end{aligned}
$$

Let $j \in\{1, \ldots, p\}$ such that $\left|u_{j}-k(t, x)\right|=\max _{i=1, \ldots, p}\left|u_{i}-k(t, x)\right|$. The previous inequalities imply that

$$
\Psi\left(t, x, \sum_{i=1}^{p} \lambda_{i} u_{i}\right) \leqslant \Psi\left(t, x, u_{j}\right) \leqslant \max _{i=1, \ldots, p} \Psi\left(t, x, u_{i}\right)
$$

Inequality (2.9) with $\rho(s):=s$ is a direct consequence of (2.29) and (2.30). 
$(a) \Rightarrow(c)$. The proof is exactly the same with the proof of implication $(b) \Rightarrow(d)$. The only additional point is that by virtue of Lemma 3.2 in [13] there exist functions $a \in K_{\infty}, \gamma \in K^{+}$such that

$$
|k(t, x)| \leqslant a(\gamma(t)|x|), \quad \forall(t, x) \in \Re^{+} \times \Re^{n} .
$$

Consequently, for every $(t, x) \in \Re^{+} \times \Re^{n}$ there exists $u \in U$ with $|u| \leqslant a(\gamma(t)|x|)$ (namely $u=k(t, x)$ ) such that (2.10) holds with $\rho(s):=s$ and $q(t) \equiv 0$. The proof is complete.

Proof of Theorem 2.9. Suppose that (1.1) admits an ORCLF which satisfies the small control property with $q(t) \equiv 0$. Define:

$$
\begin{aligned}
& \Xi(t, x, u):=\Psi(t, x, u)-\frac{1}{2} \rho(V(t, x)), \quad(t, x, u) \in \Re^{+} \times \Re^{n} \times U \\
& \Xi(t, x, u):=\Xi(0, x, u), \quad(t, x, u) \in(-1,0) \times \Re^{n} \times U .
\end{aligned}
$$

The definition of $\Xi$, given by (2.32a), (2.32b), guarantees that the function $\Xi:(-1,+\infty) \times \Re \Re^{n} \times U \rightarrow \Re \cup\{+\infty\}$ with $\Xi(t, 0,0)=0$ for all $t>-1$ is such that, for each $u \in U$ the mapping $(t, x) \rightarrow \Xi(t, x, u)$ is upper semi-continuous. By virtue of (2.10) with $q(t) \equiv 0$ and upper semi-continuity of $\Xi$, it follows that for each $(t, x) \in(-1,+\infty) \times\left(\Re^{n} \backslash\{0\}\right)$ there exist $u=u(t, x) \in U$ with $|u| \leqslant a(\gamma(\max (0, t))|x|)$ and $\delta=\delta(t, x) \in$ $(0, \min (1, t+1)), \delta(t, x) \leqslant \frac{|x|}{2}$, such that

$$
\Xi(\tau, y, u(t, x)) \leqslant 0, \forall(\tau, y) \in\left\{(\tau, y) \in(-1,+\infty) \times \Re^{n}:|\tau-t|+|y-x|<\delta\right\} .
$$

Using (2.33) and standard partition of unity arguments, we can determine sequences $\left\{\left(t_{i}, x_{i}\right) \in(-1,+\infty) \times\right.$ $\left.\left(\Re^{n} \backslash\{0\}\right)\right\}_{i=1}^{\infty},\left\{u_{i} \in U\right\}_{i=1}^{\infty},\left\{\delta_{i}\right\}_{i=1}^{\infty}$ with $\left|u_{i}\right| \leqslant a\left(\gamma\left(\max \left(0, t_{i}\right)\right)\left|x_{i}\right|\right), \delta_{i}=\delta\left(t_{i}, x_{i}\right) \in\left(0, \min \left(1, t_{i}+1\right)\right), \delta_{i}=$ $\delta\left(t_{i}, x_{i}\right) \leqslant \frac{\left|x_{i}\right|}{2}$ associated with a sequence of open sets $\left\{\Omega_{i}\right\}_{i=1}^{\infty}$ with

$$
\Omega_{i} \subseteq\left\{(\tau, y) \in(-1,+\infty) \times \Re^{n}:\left|\tau-t_{i}\right|+\left|y-x_{i}\right|<\delta_{i}\right\}
$$

forming a locally finite open covering of $(-1,+\infty) \times\left(\Re^{n} \backslash\{0\}\right)$ and in such a way that:

$$
\Xi\left(\tau, y, u_{i}\right) \leqslant 0, \quad \forall(\tau, y) \in \Omega_{i} .
$$

Also, a family of smooth functions $\left\{\theta_{i}\right\}_{i=1}^{\infty}$ with $\theta_{i}(t, x) \geqslant 0$ for all $(t, x) \in(-1,+\infty) \times\left(\Re^{n} \backslash\{0\}\right)$ can be determined with

$$
\begin{gathered}
\operatorname{supp} \theta_{i} \subseteq \Omega_{i} \\
\sum_{i=1}^{\infty} \theta_{i}(t, x)=1, \quad \forall(t, x) \in(-1,+\infty) \times\left(\Re^{n} \backslash\{0\}\right) .
\end{gathered}
$$

We define:

$$
\begin{gathered}
k(t, x):=\sum_{i=1}^{\infty} \theta_{i}(t, x) u_{i} \text { for } t \geqslant 0, x \neq 0 \\
k(t, 0):=0 \quad \text { for } \quad t \geqslant 0 .
\end{gathered}
$$

It follows from (2.38a) that $k$ is $C^{\infty}$ on the set $\Re^{+} \times\left(\Re^{n} \backslash\{0\}\right)$. Moreover, since $k(t, x)$ is defined as a (finite) convex combination of $u_{i} \in U$ and $0 \in U$, we have $k(t, x) \in U$ for all $(t, x) \in \Re^{+} \times \Re^{n}$. In order to prove continuity of $k$ at zero, let $(t, x) \in \Re^{+} \times\left(\Re^{n} \backslash\{0\}\right)$ and define $J(t, x)=\left\{j \in\{1,2, \ldots\} ; \theta_{j}(t, x) \neq 0\right\}$ (a finite set). 
Notice that for each $j \in J(t, x)$ we obtain from (2.36) that $(t, x) \in \Omega_{j}$. Consequently, using (2.34), (2.38a) and the facts $\left|u_{j}\right| \leqslant a\left(\gamma\left(\max \left(0, t_{j}\right)\right)\left|x_{j}\right|\right), \delta_{j}=\delta\left(t_{j}, x_{j}\right) \in\left(0, \min \left(1, t_{j}+1\right)\right), \delta_{j}=\delta\left(t_{j}, x_{j}\right) \leqslant \frac{\left|x_{j}\right|}{2}$, we obtain:

$$
|k(t, x)| \leqslant \max _{j \in J(t, x)}\left|u_{j}\right| \leqslant \max _{j \in J(t, x)} a\left(\gamma\left(\max \left(0, t_{j}\right)\right)\left|x_{j}\right|\right) \leqslant \tilde{a}(\tilde{\gamma}(t)|x|)
$$

where $\tilde{a}(s):=a(2 s)$ and $\tilde{\gamma}(t):=\max _{0 \leqslant \tau \leqslant t+1} \gamma(\tau)$. The above inequality in conjunction with definition (2.38b) shows continuity of $k$ at zero. Next we show that:

$$
V^{0}(t, x ; f(t, d, x, k(t, x))) \leqslant-\frac{1}{2} \rho(V(t, x)), \quad \forall(t, x, d) \in \Re^{+} \times \Re^{n} \times D .
$$

Clearly, by virtue of definition (2.38b) and inequality (2.3), it follows that inequality (2.39) holds for all $t \geqslant 0$, $x=0$. For $(t, x) \in \Re^{+} \times\left(\Re^{n} \backslash\{0\}\right)$, define $J(t, x)=\left\{j \in\{1,2, \ldots\} ; \theta_{j}(t, x) \neq 0\right\}$ (a finite set). Notice that by virtue of (2.9) and definition (2.38a) we get:

$$
\begin{aligned}
\sup _{d \in D} V^{0}(t, x ; f(t, d, x, k(t, x))) & =\sup _{d \in D} V^{0}\left(t, x ; f\left(t, d, x, \sum_{j \in J(t, x)} \theta_{j}(t, x) u_{j}\right)\right) \\
& \leqslant-\rho(V(t, x))+\max _{j \in J(t, x)}\left\{\Psi\left(t, x, u_{j}\right)\right\} .
\end{aligned}
$$

Notice that for each $j \in J(t, x)$ we obtain from (2.36) that $(t, x) \in \Omega_{j}$. Consequently, by virtue of (2.35) and definition (2.32a) we have that $\Psi\left(t, x, u_{j}\right) \leqslant \frac{1}{2} \rho(V(t, x))$, for all $j \in J(t, x)$. Combining the previous inequality with inequality (2.40), we conclude that (2.39) holds.

If the ORCLF $V$ and the function $\Psi$ involved in property (ii) of Definition 2.6 are time independent then the partition of unity arguments used above may be repeated on $\Re^{n} \backslash\{0\}$ instead of $\Re^{+} \times\left(\Re^{n} \backslash\{0\}\right)$. This implies that the constructed feedback is time invariant.

In order to show uniqueness of solutions for the closed-loop system (2.11) we consider the dynamical system

$$
\dot{x}(t)=f(t, d(t), x(t), k(t, x(t))), \quad x(t) \in \Re^{n} \backslash\{0\} .
$$

It is clear from hypothesis (H1) and smoothness of $k$ on the set $\Re^{+} \times\left(\Re^{n} \backslash\{0\}\right)$, that for every $\left(t_{0}, x_{0}, d\right) \in$ $\Re^{+} \times\left(\Re^{n} \backslash\{0\}\right) \times M_{D}$, the solution with initial condition $x\left(t_{0}\right)=x_{0} \in \Re^{n} \backslash\{0\}$, corresponding to $d \in M_{D}$ is unique and is defined on the interval $\left[t_{0}, t_{\max }\right)$, where $t_{\max }>t_{0}$ is the maximal existence time of the solution of $(2.41)$.

Notice that the solution of (2.11) with initial condition $x\left(t_{0}\right)=x_{0} \in \Re^{n} \backslash\{0\}$, corresponding to some $d \in M_{D}$ coincides with the unique solution of (2.41) evolving on $\Re^{+} \times\left(\Re^{n} \backslash\{0\}\right)$ with same initial condition $x\left(t_{0}\right)=$ $x_{0} \in \Re^{n} \backslash\{0\}$, and same $d \in M_{D}$ on the interval $\left[t_{0}, t_{\max }\right)$, where $t_{\max }>t_{0}$ is the maximal existence time of the solution of (2.41).

For the case $t_{\max }=+\infty$, uniqueness of solutions for (2.11) is a direct consequence of previous argument. Suppose next that $t_{\max }<+\infty$. To establish uniqueness of solutions for (2.11), we need the following implication, which is a consequence of (2.6) and (2.39):

$$
t_{\max }<+\infty \Rightarrow \lim _{t \rightarrow t_{\max }^{-}} x(t)=0 .
$$

In order to show (2.42), let $\left(t_{0}, x_{0}, d\right) \in \Re^{+} \times\left(\Re^{n} \backslash\{0\}\right) \times M_{D}$ and suppose that the maximal existence time $t_{\max }>t_{0}$ of the (unique) solution of (2.41) with initial condition $x\left(t_{0}\right)=x_{0} \in \Re^{n} \backslash\{0\}$ corresponding to $d \in M_{D}$ is finite, i.e., $t_{\max }<+\infty$. Lemma 2.4 in conjunction with (2.39) implies that

$$
V(t, x(t)) \leqslant V\left(t_{0}, x_{0}\right), \quad \forall t \in\left[t_{0}, t_{\max }\right) .
$$


The above inequality in conjunction with $(2.6)$ with $\beta(t) \equiv 1$ gives

$$
|x(t)| \leqslant M:=\frac{1}{\min _{\tau \in\left[0, t_{\max }\right]} \mu(\tau)} a_{1}^{-1}\left(a_{2}\left(\left|x_{0}\right|\right)\right)<+\infty, \quad \forall t \in\left[t_{0}, t_{\max }\right) .
$$

Definition of $t_{\max }$ and (2.44) implies (2.42). By applying standard arguments we may also establish that for every $\left(t_{0}, d\right) \in \Re^{+} \times M_{D}$, the solution of (2.11) with initial condition $x\left(t_{0}\right)=0$, corresponding to input $d \in M_{D}$ is unique and satisfies $x(t)=0$ for all $t \geqslant t_{\max }$. Indeed, this follows from Lemma 2.4 and inequality (2.39), which imply inequality (2.43). The previous discussion in conjunction with (2.42) asserts that the solution $x(\cdot)$ of (2.11) with initial condition $x\left(t_{0}\right)=x_{0} \in \Re^{n} \backslash\{0\}$, corresponding to $d \in M_{D}$ coincides with the solution of (2.41) with same initial condition, and same $d \in M_{D}$ on the interval $\left[t_{0}, t_{\max }\right), t_{\max }>t_{0}$ being the maximal existence time of the solution (2.41); moreover, if $t_{\max }<+\infty$, the corresponding solution of (2.11) satisfies $x(t)=0$ for all $t \geqslant t_{\max }$ and uniqueness of solutions for (2.11) is established.

The fact that (2.11) is URGAOS follows directly from Proposition 2.5 and inequality (2.39).

Finally, if there exist functions $\eta \in K^{+}, \varphi \in C^{v}(A ; U)$ where $A=\cup_{t \geqslant 0}\{t\} \times B_{t}, B_{t}=\left\{x \in \Re^{n}:|x|<4 \eta(t)\right\}$ with $\varphi(t, 0)=0$ for all $t \geqslant 0$, such that (2.12) holds, then we consider the smooth feedback defined by:

$$
k(t, x):=\left(1-h\left(\frac{|x|^{2}-2 \eta^{2}(t)}{2 \eta^{2}(t)}\right)\right) \varphi\left(t, \operatorname{Pr}_{Q(t)}(x)\right)+h\left(\frac{|x|^{2}-2 \eta^{2}(t)}{2 \eta^{2}(t)}\right) \tilde{k}(t, x)
$$

where

$$
\begin{gathered}
\tilde{k}(t, x):=\sum_{i=1}^{\infty} \theta_{i}(t, x) u_{i} \text { for } t \geqslant 0, x \neq 0 \\
\tilde{k}(t, 0):=0 \text { for } t \geqslant 0
\end{gathered}
$$

$h \in C^{\infty}(\Re ;[0,1])$ is a smooth non-decreasing function with $h(s)=0$ for all $s \leqslant 0$ and $h(s)=1$ for all $s \geqslant 1$ and $Q(t)=\left\{x \in \Re^{n}:|x| \leqslant 3 \eta(t)\right\}$. Clearly, $k$ as defined by $(2.45)$ is of class $C^{v}\left(\Re^{+} \times \Re^{n} ; U\right)$ with $k(t, 0)=0$ for all $t \geqslant 0$. Using the same arguments as in the proof of Theorem 2.8 we may establish that (2.39) holds. The proof is complete.

\section{Additional Remarks and examples on the Finite-Dimensional CASE}

The problem with Definition 2.1 of the ORCLF that might arise in practice is the assumption of the knowledge of the function $\Psi: \Re^{+} \times \Re^{n} \times U \rightarrow \Re \cup\{+\infty\}$ involved in property (ii) of Definition 2.6. Particularly, the following problem arises:

Problem (P). Consider system (1.1) under hypotheses (H1)-(H3) and the following hypothesis:

(H4) There exist a $C^{1}$ function $V: \Re^{+} \times \Re^{n} \rightarrow \Re^{+}$, which satisfies property (i) of Definition 2.6, a function $q \in \mathcal{E}$ and a $C^{0}$ positive definite function $\rho: \Re^{+} \rightarrow \Re^{+}$such that the following inequality holds:

$$
\inf _{u \in U}\left\{\frac{\partial V}{\partial t}(t, x)+\sup _{d \in D}\left(\frac{\partial V}{\partial x}(t, x) f(t, d, x, u)\right)\right\} \leqslant-\rho(V(t, x))+q(t), \quad \forall(t, x) \in \Re^{+} \times \Re^{n} .
$$

Is $V: \Re^{+} \times \Re^{n} \rightarrow \Re^{+}$an ORCLF for system (1.1)?

The proof of implication $(\mathrm{b}) \Rightarrow(\mathrm{d})$ of Theorem 2.8 gives the solution to Problem $(\mathrm{P})$ : If there exists a continuous function $k: \Re^{+} \times \Re^{n} \rightarrow U$ with $k(t, 0)=0$ for all $t \geqslant 0$, a function $\tilde{q} \in \boldsymbol{E}$ and a $C^{0}$ positive definite 
function $\tilde{\rho}: \Re^{+} \rightarrow \Re^{+}$such that

$$
\frac{\partial V}{\partial t}(t, x)+\sup _{d \in D}\left(\frac{\partial V}{\partial x}(t, x) f(t, d, x, k(t, x))\right) \leqslant-\tilde{\rho}(V(t, x))+\tilde{q}(t), \quad \forall(t, x) \in \Re^{+} \times \Re^{n}
$$

then $V$ is an ORCLF for (1.1). Particularly, the function $\Psi: \Re^{+} \times \Re^{n} \times U \rightarrow \Re \cup\{+\infty\}$ involved in property (ii) of Definition 2.6 may be defined by

$$
\begin{aligned}
\Psi(t, x, u):=\tilde{\rho}(V(t, x))+\sup \left\{\frac{\partial V}{\partial t}(t, x)+\frac{\partial V}{\partial x}(t, x) f(t, d, x, v): d \in D, v \in U\right. \\
\quad \text { with }|v-k(t, x)| \leqslant|u-k(t, x)|\} .
\end{aligned}
$$

The reader may check that $\Psi: \Re^{+} \times \Re^{n} \times U \rightarrow \Re \cup\{+\infty\}$ as defined by (3.3) satisfies inequalities (2.8), (2.9) of Definition 2.6, following exactly the same procedure as in the proof of implication (b) $\Rightarrow$ (d) of Theorem 2.8. Moreover, by virtue of Theorem 2.8, if $V: \Re^{+} \times \Re^{n} \rightarrow \Re^{+}$is an ORCLF for system (1.1) then the proof of implication $(\mathrm{d}) \Rightarrow$ (a) of Theorem 2.8 shows that there exist a continuous function $k: \Re^{+} \times \Re^{n} \rightarrow U$ with $k(t, 0)=0$ for all $t \geqslant 0$, a function $\tilde{q} \in \boldsymbol{E}$ and a $C^{0}$ positive definite function $\tilde{\rho}: \Re^{+} \rightarrow \Re^{+}$such that (3.2) holds. Consequently, $V: \Re^{+} \times \Re^{n} \rightarrow \Re^{+}$is an ORCLF for (1.1) under hypotheses (H1)-(H4) if and only if there exist a continuous function $k: \Re^{+} \times \Re^{n} \rightarrow U$ with $k(t, 0)=0$ for all $t \geqslant 0$, a function $\tilde{q} \in \boldsymbol{E}$ and a $C^{0}$ positive definite function $\tilde{\rho}: \Re^{+} \rightarrow \Re^{+}$such that (3.2) holds.

The problem with the above solution to Problem (P) is that we can check if $V: \Re^{+} \times \Re^{n} \rightarrow \Re^{+}$is an ORCLF for (1.1) by constructing a feedback stabilizer for (1.1). On the other hand, our goal in practice is to construct a desired feedback stabilizer based on the mere knowledge of the Lyapunov function $V: \Re^{+} \times \Re^{n} \rightarrow \Re^{+}$under hypotheses (H1)-(H4). Consequently, the above solution to Problem (P) is not needed for feedback construction purposes.

The rest of the section provides sufficient conditions for establishing that $V: \Re^{+} \times \Re^{n} \rightarrow \Re^{+}$under hypotheses (H1)-(H4) is an ORCLF for (1.1).

Indeed, if the mapping $u \rightarrow \frac{\partial V}{\partial x}(t, x) f(t, d, x, u)$ is quasi-convex for each fixed $(t, x, d) \in \Re^{+} \times \Re^{n} \times D$ then the mapping $u \rightarrow \frac{\partial V}{\partial t}(t, x)+\sup _{d \in D}\left(\frac{\partial V}{\partial x}(t, x) f(t, d, x, u)\right)$ is quasi-convex for each fixed $(t, x) \in \Re^{+} \times \Re^{n}$. It follows that property (ii) of Definition 2.6 is satisfied with $\Psi(t, x, u):=\rho(V(t, x))+\frac{\partial V}{\partial t}(t, x)+\sup _{d \in D}\left(\frac{\partial V}{\partial x}(t, x) f(t, d, x, u)\right)$. This is exactly the case arising in affine-in-control systems: for affine-in-control systems the mapping $u \rightarrow$ $\frac{\partial V}{\partial x}(t, x) f(t, d, x, u)$ is convex.

The following lemma helps us to generalize the above sufficient condition.

Lemma 3.1. Let the mapping $f: A \times U \rightarrow \Re$, where $U \subseteq \Re^{m}$ a closed convex set. Define the set-valued map:

$$
A \times U \ni(x, u) \rightarrow \mathcal{U}(x, u):=\overline{c o}\{v \in U: f(x, v) \leqslant f(x, u)\}
$$

and the mapping $\psi: A \times U \rightarrow \Re \cup\{+\infty\}$

$$
\psi(x, u):=\sup \{f(x, v): v \in \mathcal{U}(x, u)\} .
$$

Then for every finite set $\left\{u_{1}, u_{2}, \ldots, u_{p}\right\} \subset U$ and for every $\lambda_{i} \in[0,1](i=1, \ldots, p)$ with $\sum_{i=1}^{p} \lambda_{i}=1$, it holds that:

$$
f\left(x, \sum_{i=1}^{p} \lambda_{i} u_{i}\right) \leqslant \max \left\{\psi\left(x, u_{i}\right), i=1, \ldots, p\right\}, \quad \forall x \in A .
$$


Proof. Let a finite set $\left\{u_{1}, u_{2}, \ldots, u_{p}\right\} \subset U$ and $\lambda_{i} \in[0,1](i=1, \ldots, p)$ with $\sum_{i=1}^{p} \lambda_{i}=1$. Let $u \in\left\{u_{1}, u_{2}, \ldots, u_{p}\right\}$ with $f(x, u)=\max _{i=1, \ldots, p} f\left(x, u_{i}\right)$. It follows from definition (3.4) that $\sum_{i=1}^{p} \lambda_{i} u_{i} \in \mathcal{U}(x, u)$ and consequently, by virtue of definition (3.5), we get $f\left(x, \sum_{i=1}^{p} \lambda_{i} u_{i}\right) \leqslant \psi(x, u)$. The previous inequality combined with the fact that $u \in\left\{u_{1}, u_{2}, \ldots, u_{p}\right\}$ (which implies $\psi(x, u) \leqslant \max \left\{\psi\left(x, u_{i}\right), i=1, \ldots, p\right\}$ ) establishes (3.6). The proof is complete.

The following lemma is a direct consequence of the previous lemma.

Lemma 3.2. Let $V: \Re^{+} \times \Re^{n} \rightarrow \Re^{+}$be a $C^{1}$ function which satisfies the following properties:

(i) there exist a function $q \in \mathcal{E}$ and a $C^{0}$ positive definite function $\rho: \Re^{+} \rightarrow \Re^{+}$such that for every $(t, x) \in \Re^{+} \times \Re^{n}$ there exists $u \in U$ with $\mathcal{U}(t, x, u) \subseteq \tilde{\mathcal{U}}(t, x) \neq \emptyset$, where

$$
\mathcal{U}(t, x, u):=\overline{c o}\left\{v \in U: \sup _{d \in D}\left(\frac{\partial V}{\partial x}(t, x) f(t, d, x, v)\right) \leqslant \sup _{d \in D}\left(\frac{\partial V}{\partial x}(t, x) f(t, d, x, u)\right)\right\}
$$

and

$$
\tilde{\mathcal{U}}(t, x):=\left\{v \in U: \frac{\partial V}{\partial t}(t, x)+\sup _{d \in D}\left(\frac{\partial V}{\partial x}(t, x) f(t, d, x, v)\right) \leqslant-\rho(V(t, x))+q(t)\right\} ;
$$

(ii) for each fixed $u \in U$ the mapping $\Re^{+} \times \Re^{n} \ni(t, x) \rightarrow \sup \left\{\sup _{d \in D}\left(\frac{\partial V}{\partial x}(t, x) f(t, d, x, v)\right): v \in \mathcal{U}(t, x, u)\right\}$ is upper semi-continuous.

Then property (ii) of Definition 2.6 holds with

$$
\Psi(t, x, u):=\rho(V(t, x))+\frac{\partial V}{\partial t}(t, x)+\sup \left\{\sup _{d \in D}\left(\frac{\partial V}{\partial x}(t, x) f(t, d, x, v)\right): v \in \mathcal{U}(t, x, u)\right\} .
$$

It should be noted that if the mapping $u \rightarrow \frac{\partial V}{\partial x}(t, x) f(t, d, x, u)$ is quasi-convex for each fixed $(t, x, d) \in$ $\Re^{+} \times \Re^{n} \times D$ then the set-valued map

$$
\mathcal{U}(t, x, u):=\overline{c o}\left\{v \in U: \sup _{d \in D}\left(\frac{\partial V}{\partial x}(t, x) f(t, d, x, v)\right) \leqslant \sup _{d \in D}\left(\frac{\partial V}{\partial x}(t, x) f(t, d, x, u)\right)\right\}
$$

in property (i) of Lemma 3.2 satisfies

$$
\mathcal{U}(t, x, u)=\left\{v \in U: \sup _{d \in D}\left(\frac{\partial V}{\partial x}(t, x) f(t, d, x, v)\right) \leqslant \sup _{d \in D}\left(\frac{\partial V}{\partial x}(t, x) f(t, d, x, u)\right)\right\}
$$

and consequently property (i) of Lemma 3.2 becomes equivalent to the existence of $u \in U$ with

$$
\frac{\partial V}{\partial t}(t, x)+\sup _{d \in D}\left(\frac{\partial V}{\partial x}(t, x) f(t, d, x, u)\right) \leqslant-\rho(V(t, x))+q(t) .
$$

The following example illustrates the use of Lemma 3.2 for a special class of nonlinear systems. 
Example 3.3. Consider system (1.1) under hypotheses (H1)-(H3) with $m=1, U=\Re$ and a $C^{1}$ function $V: \Re^{+} \times \Re^{n} \rightarrow \Re^{+}$which satisfies property (i) of Definition 2.6 as well as

$$
\begin{gathered}
\frac{\partial V}{\partial t}(t, x)+\sup _{d \in D}\left(\frac{\partial V}{\partial x}(t, x) f(t, d, x, u)\right)=a(t, x) u^{2}+b(t, x) u+c(t, x), \quad \forall(t, x, u) \in \Re^{+} \times \Re^{n} \times U \\
\inf _{u \in \Re}\left(a(t, x) u^{2}+b(t, x) u+c(t, x)\right) \leqslant-\rho(V(t, x))+q(t), \quad \forall(t, x) \in \Re^{+} \times \Re^{n}
\end{gathered}
$$

for appropriate continuous mappings $a, b, c: \Re^{+} \times \Re^{n} \rightarrow \Re$ with $a(t, 0)=b(t, 0)=c(t, 0)=0$ for all $t \geqslant 0$, a function $q \in \mathcal{E}$ and a $C^{0}$ positive definite function $\rho: \Re^{+} \rightarrow \Re^{+}$. We next prove that $V: \Re^{+} \times \Re^{n} \rightarrow \Re^{+}$is an ORCLF for (1.1) provided that the following implications hold:

$$
a(t, x)<0 \quad \Rightarrow \quad-\frac{b^{2}(t, x)}{4 a(t, x)}+c(t, x) \leqslant-\rho(V(t, x))+q(t)
$$

for every sequence $\left\{\left(t_{i}, x_{i}\right)\right\}_{i=0}^{\infty}$ with $a\left(t_{i}, x_{i}\right)<0$ and $\left(t_{i}, x_{i}\right) \rightarrow(t, x)$

$$
\text { with } a(t, x)=0 \text { it holds that } \frac{b^{2}\left(t_{i}, x_{i}\right)}{\left|a\left(t_{i}, x_{i}\right)\right|} \rightarrow 0 .
$$

Following the notation of Lemma 3.2 we define $\mathcal{U}(t, x, u)=\overline{c o}\left\{v \in \Re: a(t, x) v^{2}+b(t, x) v \leqslant a(t, x) u^{2}+b(t, x) u\right\}$ and $\tilde{\mathcal{U}}(t, x)=\left\{v \in \Re: a(t, x) v^{2}+b(t, x) v+c(t, x) \leqslant-\rho(V(t, x))+q(t)\right\}$. We notice that:

(a) If $a(t, x)>0$ then for every $u \in \Re$ the set $\left\{v \in \Re: a(t, x) v^{2}+b(t, x) v \leqslant a(t, x) u^{2}+b(t, x) u\right\}$ is closed and convex and consequently, there exists $u \in \Re$ with $\mathcal{U}(t, x, u) \subseteq \tilde{\mathcal{U}}(t, x)$ (specifically, by virtue of (3.8), the inclusion $\mathcal{U}(t, x, u) \subseteq \tilde{\mathcal{U}}(t, x)$ holds for $\left.u=-\frac{b(t, x)}{2 a(t, x)}\right)$.

(b) If $a(t, x)=0$ then for every $u \in \Re$ the set $\{v \in \Re: b(t, x) v \leqslant b(t, x) u\}$ is closed and convex and consequently, there exists $u \in \Re$ with $\mathcal{U}(t, x, u) \subseteq \tilde{\mathcal{U}}(t, x)$. Specifically, if $b(t, x) \neq 0$ then the inclusion $\mathcal{U}(t, x, u) \subseteq \tilde{\mathcal{U}}(t, x)$ holds for $u=-\frac{c(t, x)+\rho(V(t, x))}{b(t, x)}$. If $b(t, x)=0$, then by virtue of (3.8), the inclusion $\mathcal{U}(t, x, u) \subseteq \tilde{\mathcal{U}}(t, x)$ holds for every $u \in \Re$.

(c) If $a(t, x)<0$ then for every $u \neq-\frac{b(t, x)}{2 a(t, x)}$ the set $\left\{v \in \Re: a(t, x) v^{2}+b(t, x) v \leqslant a(t, x) u^{2}+b(t, x) u\right\}$ is not convex and there exist $v_{1}, v_{2} \in \Re$ with $v_{1}<v_{2}$ such that

$$
\left\{v \in \Re: a(t, x) v^{2}+b(t, x) v \leqslant a(t, x) u^{2}+b(t, x) u\right\}=\left(-\infty, v_{1}\right] \cup\left[v_{2},+\infty\right) .
$$

On the other hand if $u=-\frac{b(t, x)}{2 a(t, x)}$ then it holds that

$$
\left\{v \in \Re: a(t, x) v^{2}+b(t, x) v \leqslant a(t, x) u^{2}+b(t, x) u\right\}=\Re .
$$

Consequently, if $a(t, x)<0$ then for every $u \in \Re$ it holds that $\mathcal{U}(t, x, u)=\Re$. However, in this case implication (3.9) guarantees that $\tilde{\mathcal{U}}(t, x)=\Re$ and therefore the inclusion $\mathcal{U}(t, x, u) \subseteq \tilde{\mathcal{U}}(t, x)$ holds for every $u \in \Re$.

Thus, property $(i)$ of Lemma 3.2 holds for the function $V: \Re^{+} \times \Re^{n} \rightarrow \Re^{+}$. Since

$$
\Psi(t, x, u):=\rho(V(t, x))+\frac{\partial V}{\partial t}(t, x)+\sup \left\{\sup _{d \in D}\left(\frac{\partial V}{\partial x}(t, x) f(t, d, x, v)\right): v \in \mathcal{U}(t, x, u)\right\},
$$


by virtue of all the above specifications for the set-valued map $\mathcal{U}(t, x, u)$ we get:

$$
\Psi(t, x, u):=\rho(V(t, x))+\left\{\begin{array}{cc}
a(t, x) u^{2}+b(t, x) u+c(t, x) & \text { if } \quad a(t, x) \geqslant 0 \\
-\frac{b^{2}(t, x)}{4 a(t, x)}+c(t, x) & \text { if } \quad a(t, x)<0 .
\end{array}\right.
$$

Notice that implication (3.10) guarantees that property (ii) of Lemma 3.2 holds and consequently property (ii) of Definition 2.6 holds with $\Psi$ defined by (3.11).

The reader should notice that other choices for the mapping $\Psi(t, x, u)$ are possible. For example, the selection

$$
\Psi(t, x, u):=\rho(V(t, x))+\left\{\begin{array}{ccc}
a(t, x) u^{2}+b(t, x) u+c(t, x) & \text { if } \quad a(t, x) \geqslant 0 \\
b(t, x) u+c(t, x) & \text { if } \quad a(t, x)<0
\end{array}\right.
$$

guarantees that $V: \Re^{+} \times \Re^{n} \rightarrow \Re^{+}$is an ORCLF for (1.1) provided that (3.7), (3.8) as well as the following implication holds:

$$
a(t, x)<0, b(t, x)=0 \quad \Rightarrow \quad c(t, x) \leqslant-\rho(V(t, x))+q(t) .
$$

Notice that if (3.13) holds then property (ii) of Definition 2.6 holds with $\Psi$ defined by (3.12). Moreover, notice that if implication (3.9) holds then implication (3.13) automatically holds.

The following lemma provides a "patchy" construction by combining the formula provided by Lemma 3.2 and the knowledge of appropriate functions that can be used in certain regions of $\Re^{+} \times \Re^{n}$ as feedback functions.

Lemma 3.4. Let $V: \Re^{+} \times \Re^{n} \rightarrow \Re^{+}$be a $C^{1}$ function and suppose that there exist sets $\Omega_{i} \subseteq \Re^{+} \times \Re^{n}(i=$ $0, \ldots, p)$ with $\Omega_{i} \cap \Omega_{j}=\emptyset$ for $i \neq j$ and $\underset{i=0, \ldots, p}{\cup} \Omega_{i}=\Re^{+} \times \Re^{n}$, functions $k_{i}: \Omega_{i} \rightarrow U(i=1, \ldots, p)$, a function $q \in \boldsymbol{E}$ and $a C^{0}$ positive definite function $\rho: \Re^{+} \rightarrow \Re^{+}$such that:

(i) for every $(t, x) \in \Omega_{0}$ there exists $u \in U$ with $\mathcal{U}(t, x, u) \subseteq \tilde{\mathcal{U}}(t, x) \neq \emptyset$, where

$$
\mathcal{U}(t, x, u):=\overline{c o}\left\{v \in \mathcal{U}: \sup _{d \in D}\left(\frac{\partial V}{\partial x}(t, x) f(t, d, x, v)\right) \leqslant \sup _{d \in D}\left(\frac{\partial V}{\partial x}(t, x) f(t, d, x, u)\right)\right\}
$$

and

$$
\tilde{\mathcal{U}}(t, x):=\left\{v \in U: \frac{\partial V}{\partial t}(t, x)+\sup _{d \in D}\left(\frac{\partial V}{\partial x}(t, x) f(t, d, x, v)\right) \leqslant-\rho(V(t, x))+q(t)\right\} ;
$$

(ii) for every $i=1, \ldots, p$ and $(t, x) \in \Omega_{i}$ it holds that

$$
\frac{\partial V}{\partial t}(t, x)+\sup _{d \in D}\left(\frac{\partial V}{\partial x}(t, x) f\left(t, d, x, k_{i}(t, x)\right)\right) \leqslant-\rho(V(t, x))+q(t) .
$$

Consider the function $\Psi: \Re^{+} \times \Re^{n} \times U \rightarrow \Re \cup\{+\infty\}$ defined by:

$$
\begin{gathered}
\Psi(t, x, u):=\rho(V(t, x))+\frac{\partial V}{\partial t}(t, x)+\sup \left\{\sup _{d \in D}\left(\frac{\partial V}{\partial x}(t, x) f(t, d, x, v)\right): v \in \mathcal{U}(t, x, u)\right\}, \text { for }(t, x) \in \Omega_{0} \\
\Psi(t, x, u):=\rho(V(t, x))+\sup \left\{\frac{\partial V}{\partial t}(t, x)+\frac{\partial V}{\partial x}(t, x) f(t, d, x, v): d \in D, v \in U\right. \\
\text { with } \left.\left|v-k_{i}(t, x)\right| \leqslant\left|u-k_{i}(t, x)\right|\right\}, \text { for }(t, x) \in \Omega_{i}, i=1, \ldots, p
\end{gathered}
$$

and suppose that $\Psi: \Re^{+} \times \Re^{n} \times U \rightarrow \Re \cup\{+\infty\}$ is upper semi-continuous.

Then property (ii) of Definition 2.6 holds with $\Psi: \Re^{+} \times \Re^{n} \times U \rightarrow \Re \cup\{+\infty\}$ defined by (3.14). 
The following example illustrates the efficiency of Lemma 3.4. It shows that the knowledge of appropriate functions that can be used in certain regions of $\Re^{+} \times \Re^{n}$ as feedback functions, helps us to obtain less conservative results.

Example 3.5. Consider system (1.1) under hypotheses (H1)-(H3) with $m=1, U=\Re$ and a $C^{1}$ function $V: \Re^{+} \times \Re^{n} \rightarrow \Re^{+}$which satisfies property (i) of Definition 2.6 as well as (3.7), (3.8) for appropriate continuous mappings $a, b, c: \Re^{+} \times \Re^{n} \rightarrow \Re$ with $a(t, 0)=b(t, 0)=c(t, 0)=0$ for all $t \geqslant 0$, a function $q \in \boldsymbol{E}$ and a $C^{0}$ positive definite function $\rho: \Re^{+} \rightarrow \Re^{+}$. We showed in Example 3.3 that $V: \Re^{+} \times \Re^{n} \rightarrow \Re^{+}$is an ORCLF for (1.1) provided that implications (3.9), (3.10) hold. In this example we show that implication (3.10) only is sufficient to guarantee that $V: \Re^{+} \times \Re^{n} \rightarrow \Re^{+}$is an ORCLF for (1.1). Indeed, let $\Omega_{0}:=\left\{(t, x) \in \Re^{+} \times \Re^{n}: a(t, x) \geqslant 0\right\}$ and $\Omega_{1}:=\left\{(t, x) \in \Re^{+} \times \Re^{n}: a(t, x)<0\right\}$. Moreover, define $k_{1}: \Omega_{1} \rightarrow \Re$ by

$$
k_{1}(t, x):=\frac{\sqrt{b^{2}(t, x)+4|a(t, x) c(t, x)|+4|a(t, x)| \rho(V(t, x))}-b(t, x)}{2 a(t, x)}, \quad(t, x) \in \Omega_{1} .
$$

The specification of the set-valued map $\mathcal{U}(t, x, u)$ for $(t, x) \in \Omega_{0}$ has been given in Example 3.3. Therefore, the function $\Psi: \Re^{+} \times \Re^{n} \times U \rightarrow \Re \cup\{+\infty\}$ involved in Lemma 3.4 is defined by:

$$
\begin{gathered}
\Psi(t, x, u):=\rho(V(t, x))+a(t, x) u^{2}+b(t, x) u+c(t, x), \text { for }(t, x) \in \Omega_{0} \\
\Psi(t, x, u):=\rho(V(t, x))+\sup \left\{a(t, x) v^{2}+b(t, x) v+c(t, x):\left|v-k_{1}(t, x)\right| \leqslant\left|u-k_{1}(t, x)\right|\right\}, \text { for }(t, x) \in \Omega_{1} .
\end{gathered}
$$

Clearly, $\Psi(t, x, u)$ as defined by (3.16a) is continuous on the interior of $\Omega_{0}$. Furthermore, it follows from continuity of $a(t, x), b(t, x), c(t, x), k_{1}(t, x)$ on $\Omega_{1}$ and Theorem 1.4.16 in [7] that $\Psi(t, x, u)$ as defined by (3.16b) is upper semi-continuous on $\Omega_{1}$. The reader should notice that implication (3.10) guarantees that $\Psi: \Re^{+} \times \Re^{n} \times$ $U \rightarrow \Re \cup\{+\infty\}$ is upper semi-continuous (since $\Psi(t, x, u) \leqslant \rho(V(t, x))-\frac{b^{2}(t, x)}{4 a(t, x)}+c(t, x)$ for all $\left.(t, x, u) \in \Omega_{1} \times \Re\right)$. Consequently, Lemma 3.4 guarantees that property (ii) of Definition 2.6 holds with $\Psi: \Re^{+} \times \Re^{n} \times U \rightarrow \Re \cup\{+\infty\}$ as defined by (3.16) and that $V: \Re^{+} \times \Re^{n} \rightarrow \Re^{+}$is an ORCLF for (1.1).

\section{EXTENSIONS TO SYSTEMS DESCRIBED BY RETARDED FUNCTIONAL DIFFERENTIAL EQUATIONS}

In this section we extend the methodology presented in Section 2, to infinite-dimensional systems described by Retarded Functional Differential Equations (RFDEs). Particularly, we consider control systems of the form (1.3) under the following hypotheses:

(S1) The mapping $(x, u, d) \rightarrow f(t, d, x, u)$ is continuous for each fixed $t \geqslant 0$ and such that for every bounded $I \subseteq \Re^{+}$and for every bounded $S \subset C^{0}\left([-r, 0] ; \Re^{n}\right) \times U$, there exists a constant $L \geqslant 0$ such that:

$$
\begin{aligned}
& (x(0)-y(0))^{\prime}(f(t, d, x, u)-f(t, d, y, u)) \leqslant L \max _{\tau \in[-r, 0]}|x(\tau)-y(\tau)|^{2}=L\|x-y\|_{r}^{2} \\
& \forall t \in I, \forall(x, u, y, u) \in S \times S, \forall d \in D .
\end{aligned}
$$

Hypothesis (S1) is equivalent to the existence of a continuous non-decreasing function $L: \Re^{+} \rightarrow \Re^{+}$, with the following property:

$$
\begin{aligned}
& (x(0)-y(0))^{\prime}(f(t, d, x, u)-f(t, d, y, u)) \leqslant L\left(t+\|x\|_{r}+\|y\|_{r}+|u|\right)\|x-y\|_{r}^{2} \\
& \forall(t, x, y, d, u) \in \Re^{+} \times C^{0}\left([-r, 0] ; \Re^{n}\right) \times C^{0}\left([-r, 0] ; \Re^{n}\right) \times D \times U .
\end{aligned}
$$

(S2) For every bounded $\Omega \subset \Re^{+} \times D \times C^{0}\left([-r, 0] ; \Re^{n}\right) \times U$ the image set $f(\Omega) \subset \Re^{n}$ is bounded. 
(S3) There exists a countable set $A \subset \Re^{+}$, which is either finite or $A=\left\{t_{k} ; k=1, \ldots, \infty\right\}$ with $t_{k+1}>t_{k}>0$ for all $k=1,2, \ldots$ and $\lim t_{k}=+\infty$, such that mapping $(t, x, u, d) \in\left(\Re^{+} \backslash A\right) \times C^{0}\left([-r, 0] ; \Re^{n}\right) \times U \times D \rightarrow f(t, d, x, u)$ is continuous. Moreover, for each fixed $\left(t_{0}, x, u, d\right) \in \Re^{+} \times C^{0}\left([-r, 0] ; \Re^{n}\right) \times U \times D$, we have $\lim _{t \rightarrow t_{0}^{+}} f(t, d, x, u)=$ $f\left(t_{0}, d, x, u\right)$.

(S4) For every $\varepsilon>0, t \in \Re^{+}$, there exists $\delta:=\delta(\varepsilon, t)>0$ such that $\sup \left\{|f(\tau, d, x, u)| ; \tau \in \Re^{+}, d \in D, u \in U\right.$, $\left.|\tau-t|+\|x\|_{r}+|u|<\delta\right\}<\varepsilon$.

(S5) The mapping $u \rightarrow f(t, d, x, u)$ is Lipschitz on bounded sets, in the sense that for every bounded $I \subseteq \Re^{+}$ and for every bounded $S \subset C^{0}\left([-r, 0] ; \Re^{n}\right) \times U$, there exists a constant $L_{U} \geqslant 0$ such that:

$$
|f(t, d, x, u)-f(t, d, x, v)| \leqslant L_{U}|u-v|, \forall t \in I, \forall(x, u, x, v) \in S \times S, \forall d \in D .
$$

Hypothesis (S5) is equivalent to the existence of a continuous, non-decreasing function $L_{U}: \Re^{+} \rightarrow \Re^{+}$, with the following property:

$$
\begin{aligned}
& |f(t, d, x, u)-f(t, d, x, v)| \leqslant L_{U}\left(t+\|x\|_{r}+|u|+|v|\right)|u-v| \\
& \forall(t, x, d, u, v) \in \Re^{+} \times C^{0}\left([-r, 0] ; \Re^{n}\right) \times D \times U \times U .
\end{aligned}
$$

(S6) The set $D \subset \Re^{l}$ is compact and $U \subseteq \Re^{m}$ is a closed convex set.

(S7) The mapping $H(t, x)$ is Lipschitz on bounded sets, in the sense that for every bounded $I \subseteq \Re^{+}$and for every bounded $S \subset C^{0}\left([-r, 0] ; \Re^{n}\right)$, there exists a constant $L_{H} \geqslant 0$ such that:

$$
\|H(t, x)-H(\tau, y)\|_{\mathcal{Y}} \leqslant L_{H}\left(|t-\tau|+\|x-y\|_{r}\right), \forall(t, \tau) \in I \times I, \forall(x, y) \in S \times S .
$$

Following the methodology described in Section 2, we next analyze in detail the following steps of the method:

4.1. Notions of output stability

4.2. Lyapunov-like criteria for output stability

4.3. Definition of the output robust control Lyapunov functional

4.4. Converse Lyapunov theorems for output stability.

\subsection{Notions of output stability}

We consider uncertain dynamical systems described by RFDEs of the form:

$$
\begin{aligned}
& \dot{x}(t)=f\left(t, d(t), T_{r}(t) x\right), t \geqslant t_{0} \\
& Y(t)=H\left(t, T_{r}(t) x\right) \\
& x(t) \in \Re^{n}, Y(t) \in Y, d(t) \in D
\end{aligned}
$$

where $r>0$ is a constant, $f: \Re^{+} \times D \times C^{0}\left([-r, 0] ; \Re^{n}\right) \rightarrow \Re^{n}, H: \Re^{+} \times C^{0}\left([-r, 0] ; \Re^{n}\right) \rightarrow \mathcal{Y}$ satisfy $f(t, d, 0)=0, H(t, 0)=0$ for all $(t, d) \in \Re^{+} \times D, D \subseteq \Re^{l}$ is a non-empty compact set, $\mathcal{Y}$ is a normed linear space and $T_{r}(t) x=x(t+\theta) ; \theta \in[-r, 0]$, under the following hypotheses:

(R1) The mapping $(x, d) \rightarrow f(t, d, x)$ is continuous for each fixed $t \geqslant 0$ and such that for every bounded $I \subseteq \Re^{+}$ and for every bounded $S \subset C^{0}\left([-r, 0] ; \Re^{n}\right)$, there exists a constant $L \geqslant 0$ such that

$$
\begin{aligned}
& (x(0)-y(0))^{\prime}(f(t, d, x)-f(t, d, y)) \leqslant L\|x-y\|_{r}^{2} \\
& \forall t \in I, \forall(x, y) \in S \times S, \forall d \in D .
\end{aligned}
$$

(R2) For every bounded $\Omega \subset \Re^{+} \times D \times C^{0}\left([-r, 0] ; \Re^{n}\right)$ the image set $f(\Omega) \subset \Re^{n}$ is bounded. 
(R3) There exists a countable set $A \subset \Re^{+}$, which is either finite or $A=\left\{t_{k} ; k=1, \ldots, \infty\right\}$ with $t_{k+1}>t_{k}>0$ for all $k=1,2, \ldots$ and $\lim t_{k}=+\infty$, such that mapping $(t, x, d) \in\left(\Re^{+} \backslash A\right) \times C^{0}\left([-r, 0] ; \Re^{n}\right) \times D \rightarrow f(t, d, x)$ is continuous. Moreover, for each fixed $\left(t_{0}, x, d\right) \in \Re^{+} \times C^{0}\left([-r, 0] ; \Re^{n}\right) \times D$, we have $\lim _{t \rightarrow t_{0}^{+}} f(t, d, x)=f\left(t_{0}, d, x\right)$.

(R4) Hypothesis (S7) holds for the output map.

It should be emphasized for systems of the form (4.3) under hypotheses (R1)-(R4) that

$-0 \in C^{0}\left([-r, 0] ; \Re^{n}\right)$ is a robust equilibrium point in the sense described in $[13,16]$ for system (4.3) under hypotheses (R1)-(R4);

- system (4.3) under hypotheses (R1)-(R4) satisfies the "Boundedness-Implies-Continuation" property and the classical semigroup property (see $[13,16])$;

- for each $\left(t_{0}, x_{0}, d\right) \in \Re^{+} \times C^{0}\left([-r, 0] ; \Re^{n}\right) \times M_{D}$ there exist $t_{\max } \in\left(t_{0},+\infty\right]$ and a unique continuous mapping $x:\left[t_{0}-r, t_{\max }\right) \rightarrow \Re^{n}$ (the solution of (4.3)) being absolutely continuous on $\left[t_{0}, t_{\max }\right)$ with $x\left(t_{0}+\theta\right)=x_{0}(\theta)$ for all $\theta \in[-r, 0]$ and $\dot{x}(t)=f\left(t, d(t), T_{r}(t) x\right)$ a.e. for $t \in\left[t_{0}, t_{\max }\right)$. Moreover, if $t_{\max }<+\infty$ then $\lim \sup |x(t)|=+\infty$. We denote the solution of (4.3) with initial condition $T_{r}\left(t_{0}\right) x=x_{0}$ $t \rightarrow t_{\max }^{-}$

corresponding to $d \in M_{D}$ by $x\left(t, t_{0}, x_{0}, d\right) \in C^{0}\left([-r, 0] ; \Re^{n}\right)$ and by $Y\left(t, t_{0}, x_{0}, d\right)$ we denote the output of system (4.3), i.e., $Y\left(t, t_{0}, x_{0}, d\right)=H\left(t, x\left(t, t_{0}, x_{0}, d\right)\right)$.

For systems of the form (4.3) under hypotheses (R1)-(R4) we adopt the definitions of RGAOS and URGAOS given in [16] for a wide class of deterministic systems with disturbances. For completeness we repeat the definitions here.

Definition 4.1. We say that (4.3) under hypotheses (R1)-(R4) is Robustly Forward Complete (RFC) if for every $s \geqslant 0, T \geqslant 0$, it holds that

$$
\sup \left\{\left\|x\left(t_{0}+\xi, t_{0}, x_{0}, d\right)\right\|_{r} ; \quad \xi \in[0, T],\left\|x_{0}\right\|_{r} \leqslant s, t_{0} \in[0, T], d \in M_{D}\right\}<+\infty .
$$

Definition 4.2. Consider system (4.3) under hypotheses (R1)-(R4). We say that (4.3) is Robustly Globally Asymptotically Output Stable (RGAOS), if (4.3) is RFC and the following properties hold:

P1 (Output Stability). For every $\varepsilon>0, T \geqslant 0$, it holds that

$$
\sup \left\{\left\|Y\left(t, t_{0}, x_{0}, d\right)\right\|_{\mathcal{Y}} ; t \geqslant t_{0},\left\|x_{0}\right\|_{r} \leqslant \varepsilon, t_{0} \in[0, T], d \in M_{D}\right\}<+\infty
$$

and there exists a $\delta:=\delta(\varepsilon, T)>0$ such that

$$
\left\|x_{0}\right\|_{r} \leqslant \delta, t_{0} \in[0, T] \Rightarrow\left\|Y\left(t, t_{0}, x_{0}, d\right)\right\|_{\mathcal{Y}} \leqslant \varepsilon, \quad \forall t \geqslant t_{0}, \quad \forall d \in M_{D} .
$$

P2 (Uniform Output Attractivity on bounded sets of initial data). For every $\varepsilon>0, T \geqslant 0$ and $R \geqslant 0$, there exists a $\tau:=\tau(\varepsilon, T, R) \geqslant 0$, such that

$$
\left\|x_{0}\right\|_{r} \leqslant R, t_{0} \in[0, T] \Rightarrow\left\|Y\left(t, t_{0}, x_{0}, d\right)\right\|_{\mathcal{Y}} \leqslant \varepsilon, \quad \forall t \geqslant t_{0}+\tau, \quad \forall d \in M_{D} .
$$

Definition 4.3. Consider system (4.3) under hypotheses (R1)-(R4). We say that (4.3) is Uniformly Robustly Globally Asymptotically Output Stable (URGAOS), if (4.3) is RFC and the following properties hold:

P1 (Uniform Output Stability). For every $\varepsilon>0$, it holds that

$$
\sup \left\{\left\|Y\left(t, t_{0}, x_{0}, d\right)\right\|_{\mathcal{Y}} ; t \geqslant t_{0},\left\|x_{0}\right\|_{r} \leqslant \varepsilon, t_{0} \geqslant 0, d \in M_{D}\right\}<+\infty
$$

and there exists a $\delta:=\delta(\varepsilon)>0$ such that

$$
\left\|x_{0}\right\|_{r} \leqslant \delta, t_{0} \geqslant 0 \Rightarrow\left\|Y\left(t, t_{0}, x_{0}, d\right)\right\|_{\mathcal{Y}} \leqslant \varepsilon, \quad \forall t \geqslant t_{0}, \quad \forall d \in M_{D} .
$$


P2 (Uniform Output Attractivity on bounded sets of initial states). For every $\varepsilon>0$ and $R \geqslant 0$, there exists a $\tau:=\tau(\varepsilon, R) \geqslant 0$, such that

$$
\left\|x_{0}\right\|_{r} \leqslant R, t_{0} \geqslant 0 \Rightarrow\left\|Y\left(t, t_{0}, x_{0}, d\right)\right\|_{\mathcal{Y}} \leqslant \varepsilon, \quad \forall t \geqslant t_{0}+\tau, \quad \forall d \in M_{D} .
$$

Obviously, the notions of RGAOS, URGAOS are direct extensions of the notions of RGAOS and URGAOS for finite-dimensional systems.

\subsection{Lyapunov-like criteria for output stability}

Let $x \in C^{0}\left([-r, 0] ; \Re^{n}\right)$ and $V: \Re^{+} \times C^{0}\left([-r, 0] ; \Re^{n}\right) \rightarrow \Re$ be a locally bounded functional. By $E_{h}(x ; v)$, where $0 \leqslant h<r$ and $v \in \Re^{n}$ we denote the following operator:

$$
E_{h}(x ; v):=\left\{\begin{array}{cll}
x(0)+(\theta+h) v & \text { for } & -h<\theta \leqslant 0 \\
x(\theta+h) & \text { for } \quad-r \leqslant \theta \leqslant-h
\end{array}\right.
$$

and we define

$$
V^{0}(t, x ; v):=\limsup _{\substack{h \rightarrow 0^{+} \\ y \rightarrow 0, y \in C^{0}\left([-r, 0] ; \Re^{n}\right)}} \frac{V\left(t+h, E_{h}(x ; v)+h y\right)-V(t, x)}{h} .
$$

The following lemma presents some elementary properties of the generalized derivative given above. Notice that the function $(t, x, v) \rightarrow V^{0}(t, x ; v)$ may take values in the extended real number set $\Re^{*}=[-\infty,+\infty]$. Its proof is almost identical with Lemma 2.7 in [15] and is omitted.

Lemma 4.4. Let $V: \Re^{+} \times C^{0}\left([-r, 0] ; \Re^{n}\right) \rightarrow \Re$ be a locally bounded functional and let $x \in C^{0}\left(\left[t_{0}-r, t_{\max }\right) ; \Re^{n}\right)$ a solution of (4.3) under hypotheses $(\mathrm{R} 1)-(\mathrm{R} 4)$ with initial condition $x\left(t_{0}\right)=x_{0} \in C^{0}\left([-r, 0] ; \Re^{n}\right)$, corresponding to certain $d \in M_{D}$, where $t_{\max } \in\left(t_{0},+\infty\right]$ is the maximal existence time of the solution. Then it holds that

$$
\limsup _{h \rightarrow 0^{+}} h^{-1}\left(V\left(t+h, T_{r}(t+h) x\right)-V\left(t, T_{r}(t) x\right)\right) \leqslant V^{0}\left(t, T_{r}(t) x ; D^{+} x(t)\right) \text {, a.e. on }\left[t_{0}, t_{\max }\right)
$$

where $D^{+} x(t)=\lim _{h \rightarrow 0^{+}} h^{-1}(x(t+h)-x(t))$.

An important class of functionals is presented next.

Definition 4.5. We say that a continuous functional $V: \Re^{+} \times C^{0}\left([-r, 0] ; \Re^{n}\right) \rightarrow \Re^{+}$, is "almost Lipschitz on bounded sets", if there exist non-decreasing functions $L_{V}: \Re^{+} \rightarrow \Re^{+}, P: \Re^{+} \rightarrow \Re^{+}, G: \Re^{+} \rightarrow[1,+\infty)$ such that for all $R \geqslant 0$, the following properties hold:

(P1) For every $x, y \in\left\{x \in C^{0}\left([-r, 0] ; \Re^{n}\right) ;\|x\|_{r} \leqslant R\right\}$, it holds that:

$$
|V(t, y)-V(t, x)| \leqslant L_{V}(R)\|y-x\|_{r}, \quad \forall t \in[0, R]
$$

(i.e., the mapping $\Re^{+} \times C^{0}\left([-r, 0] ; \Re^{n}\right) \ni(t, x) \rightarrow V(t, x) \in \Re^{+}$is completely locally Lipschitz with respect to $\left.x \in C^{0}\left([-r, 0] ; \Re^{n}\right)\right)$.

(P2) For every absolutely continuous function $x:[-r, 0] \rightarrow \Re^{n}$ with $\|x\|_{r} \leqslant R$ and essentially bounded derivative, it holds that:

$$
|V(t+h, x)-V(t, x)| \leqslant h P(R)\left(1+\sup _{-r \leqslant \tau \leqslant 0}|\dot{x}(\tau)|\right) \text {, for all } t \in[0, R] \text { and } 0 \leqslant h \leqslant \frac{1}{G\left(R+\sup _{-r \leqslant \tau \leqslant 0}|\dot{x}(\tau)|\right)} .
$$


The reader should notice that for functionals $V: \Re^{+} \times C^{0}\left([-r, 0] ; \Re^{n}\right) \rightarrow \Re^{+}$, which are almost Lipschitz on bounded sets we obtain the following simplification for the derivative $V^{0}(t, x ; v)$ defined by (4.5) for all $(t, x, v) \in \Re^{+} \times C^{0}\left([-r, 0] ; \Re^{n}\right) \times \Re^{n}:$

$$
V^{0}(t, x ; v)=\limsup _{h \rightarrow 0^{+}} \frac{V\left(t+h, E_{h}(x ; v)\right)-V(t, x)}{h} .
$$

The following proposition is based on the results obtained in [20] and provides Lyapunov-like criteria for RGAOS and URGAOS for (4.3). Its proof is provided in Appendix.

Proposition 4.6. Consider system (4.3) under hypotheses (R1)-(R4). Suppose that there exist functions $a_{1}, a_{2} \in K_{\infty}, \beta, \mu \in K^{+}, q \in \mathcal{E}$, a positive definite continuous function $\rho: \Re^{+} \rightarrow \Re^{+}$and a mapping $V: \Re^{+} \times C^{0}\left([-r, 0] ; \Re^{n}\right) \rightarrow \Re^{+}$, which is almost Lipschitz on bounded sets, such that the following inequalities hold for all $(t, x, d) \in \Re^{+} \times C^{0}\left([-r, 0] ; \Re^{n}\right) \times D$ :

$$
\begin{gathered}
\max \left\{a_{1}\left(\|H(t, x)\|_{\mathcal{Y}}\right), a_{1}\left(\mu(t)\|x\|_{r}\right)\right\} \leqslant V(t, x) \leqslant a_{2}\left(\beta(t)\|x\|_{r}\right) \\
V^{0}(t, x ; f(t, d, x)) \leqslant-\rho(V(t, x))+q(t) .
\end{gathered}
$$

Then system (4.3) is RGAOS. Moreover, if $\beta(t) \equiv 1$ and $q(t) \equiv 0$ then system (4.3) is URGAOS.

\subsection{Definition of the Output Robust Control Lyapunov Functional}

We next give the definition of the Output Robust Control Lyapunov Functional for system (1.3). The definition is in the same spirit with Definition 2.6 of the notion of ORCLF for finite-dimensional control systems.

Definition 4.7. We say that (1.3) admits an Output Robust Control Lyapunov Functional (ORCLF) if there exists an almost Lipschitz on bounded sets functional $V: \Re^{+} \times C^{0}\left([-r, 0] ; \Re^{n}\right) \rightarrow \Re^{+}$, referred to as ORCLF, which satisfies the following properties:

(i) There exist functions $a_{1}, a_{2} \in K_{\infty}, \beta, \mu \in K^{+}$such that inequality (4.7) holds for all $(t, x) \in$ $\Re^{+} \times C^{0}\left([-r, 0] ; \Re^{n}\right)$.

(ii) There exists a function $\Psi: \Re^{+} \times \Re^{p} \times U \rightarrow \Re \cup\{+\infty\}$ with $\Psi(t, 0,0)=0$ for all $t \geqslant 0$ such that for each $u \in U$ the mapping $(t, \varphi) \rightarrow \Psi(t, \varphi, u)$ is upper semi-continuous, a function $q \in \mathcal{E}$, a continuous mapping $\Re^{+} \times C^{0}\left([-r, 0] ; \Re^{n}\right)$ э $(t, x) \rightarrow \Phi(t, x) \in \Re^{p}$ being completely locally Lipschitz with respect to $x \in C^{0}\left([-r, 0] ; \Re^{n}\right)$ with $\Phi(t, 0)=0$ for all $t \geqslant 0$ and a $C^{0}$ positive definite function $\rho: \Re^{+} \rightarrow \Re^{+}$ such that the following inequality holds:

$$
\inf _{u \in U} \Psi(t, \varphi, u) \leqslant q(t), \quad \forall t \geqslant 0, \forall \varphi=\left(\varphi_{1}, \ldots, \varphi_{p}\right)^{\prime} \in \Re^{p} .
$$

Moreover, for every finite set $\left\{u_{1}, u_{2}, \ldots, u_{N}\right\} \subset U$ and for every $\lambda_{i} \in[0,1](i=1, \ldots, N)$ with $\sum_{i=1}^{N} \lambda_{i}=1$, it holds that:

$$
\begin{gathered}
\sup _{d \in D} V^{0}\left(t, x ; f\left(t, d, x, \sum_{i=1}^{N} \lambda_{i} u_{i}\right)\right) \leqslant-\rho(V(t, x))+\max \left\{\Psi\left(t, \Phi(t, x), u_{i}\right), i=1, \ldots, N\right\}, \\
\forall(t, x) \in \Re^{+} \times C^{0}\left([-r, 0] ; \Re^{n}\right) .
\end{gathered}
$$

If in addition to the above there exist $a \in K_{\infty}, \gamma \in K^{+}$such that for every $(t, \varphi) \in \Re^{+} \times \Re^{p}$ there exists $u \in U$ with $|u| \leqslant a(\gamma(t)|\varphi|)$ and

$$
\Psi(t, \varphi, u) \leqslant q(t)
$$

then we say that $V: \Re^{+} \times C^{0}\left([-r, 0] ; \Re^{n}\right) \rightarrow \Re^{+}$satisfies the "small-control" property. 
For the case $H(t, x) \equiv x \in C^{0}\left([-r, 0] ; \Re^{n}\right)$ we simply call $V: \Re^{+} \times C^{0}\left([-r, 0] ; \Re^{n}\right) \rightarrow \Re^{+}$a State Robust Control Lyapunov Functional (SRCLF).

Remark 4.8. It should be clear that in the finite-dimensional case the continuous mapping $\Phi(t, x)=$ $\left(\Phi_{1}(t, x), \ldots, \Phi_{p}(t, x)\right)^{\prime}$ is replaced by the mapping $\Phi(t, x):=x \in \Re^{n}$ with $p=n$. The question of the construction of the mapping $\Psi: \Re^{+} \times \Re^{p} \times U \rightarrow \Re \cup\{+\infty\}$ can be handled in exactly the same way as shown in Section 3, provided that we can find appropriate continuous mappings $\Phi(t, x)=\left(\Phi_{1}(t, x), \ldots, \Phi_{p}(t, x)\right)^{\prime}, G$ : $\Re^{+} \times \Re^{p} \times U \rightarrow \Re$ with $\rho(V(t, x))+\sup _{d \in D} V^{0}(t, x ; f(t, d, x, u)) \leqslant G(t, \Phi(t, x), u)$ for all $(t, x) \in \Re^{+} \times C^{0}\left([-r, 0] ; \Re^{n}\right)$ and $\inf _{u \in U} G(t, \varphi, u) \leqslant q(t)$ for all $(t, \varphi) \in \Re^{+} \times \Re^{p}$. In this case all constructions of $\Psi: \Re^{+} \times \Re^{p} \times U \rightarrow \Re \cup\{+\infty\}$ given in Section 3 may be repeated with the quantity $\frac{\partial V}{\partial t}(t, x)+\sup _{d \in D}\left(\frac{\partial V}{\partial x}(t, x) f(t, d, x, u)\right)+\rho(V(t, x))$ replaced by the quantity $G(t, \varphi, u)$.

\subsection{Converse Lyapunov theorems for output stability}

In the rest of Section 4, we are going to exploit the converse Lyapunov theorems for RGAOS and URGAOS presented in [20].

\subsection{Main results}

We are now in a position to state and prove our main results for the infinite-dimensional case (1.3).

Theorem 4.9. Consider system (1.3) under hypotheses (S1)-(S7). The following statements are equivalent:

(a) There exists a continuous mapping $k: \Re^{+} \times C^{0}\left([-r, 0] ; \Re^{n}\right) \ni(t, x) \rightarrow k(t, x) \in U$ being completely locally Lipschitz with respect to $x \in C^{0}\left([-r, 0] ; \Re^{n}\right)$ with $k(t, 0)=0$ for all $t \geqslant 0$, such that the closedloop system (1.3) with $u=k\left(t, T_{r}(t) x\right)$ is $R G A O S$.

(b) System (1.3) admits an ORCLF, which satisfies the small control property with $q(t) \equiv 0$.

(c) System (1.3) admits an ORCLF.

Theorem 4.10. Consider system (1.3) under hypotheses (S1)-(S7). The following statements are equivalent:

(a) System (1.3) admits an ORCLF, which satisfies the small-control property and inequalities (4.7), (4.11) with $\beta(t) \equiv 1, q(t) \equiv 0$. Moreover, there exist continuous mappings $\eta \in K^{+}, A \ni(t, \varphi) \rightarrow K(t, \varphi) \in U$ where $A=\cup_{t \geqslant 0}\{t\} \times\left\{\varphi \in \Re^{p}:|\varphi|<4 \eta(t)\right\}$ being locally Lipschitz with respect to $\varphi$ with $K(t, 0)=0$ for all $t \geqslant 0$ and such that

$\Psi(t, \Phi(t, x), K(t, \Phi(t, x))) \leqslant 0$, for all $(t, x) \in \Re^{+} \times C^{0}\left([-r, 0] ; \Re^{n}\right)$ with $|\Phi(t, x)| \leqslant 2 \eta(t)$

where $\Phi=\left(\Phi_{1}, \ldots, \Phi_{p}\right)^{\prime}: \Re^{+} \times C^{0}\left([-r, 0] ; \Re^{n}\right) \rightarrow \Re^{p}$ and $\Psi: \Re^{+} \times \Re^{p} \times U \rightarrow \Re \cup\{+\infty\}$ are the mappings involved in property (ii) of Definition 4.7.

(b) There exists a continuous mapping $k: \Re^{+} \times C^{0}\left([-r, 0] ; \Re^{n}\right)$ э $(t, x) \rightarrow k(t, x) \in U$ being completely locally Lipschitz with respect to $x \in C^{0}\left([-r, 0] ; \Re^{n}\right)$ with $k(t, 0)=0$ for all $t \geqslant 0$, such that the closedloop system (1.3) with $u=k\left(t, T_{r}(t) x\right)$ is URGAOS.

Remark 4.11. From the proof of Theorem 4.10 it will become apparent that if statement (a) of Theorem 4.10 is strengthened so that the ORCLF $V$, the mappings $\Phi=\left(\Phi_{1}, \ldots, \Phi_{p}\right)^{\prime}: \Re^{+} \times C^{0}\left([-r, 0] ; \Re^{n}\right) \rightarrow \Re^{p}$, $\Psi$ involved in property (ii) of Definition 4.7 and the mapping $K: A \rightarrow U$ are time independent then the continuous mapping $k$, whose existence is guaranteed by statement (b) of Theorem 4.10, is time invariant. Moreover, the proof of Theorem 4.9 shows that the feedback in statement (a) of Theorem 4.9 is actually a $C^{\infty}$ function of $t$ and $\Phi(t, x)$; the reader should notice the analogy with Theorem 2.8 (in the finite-dimensional case $\Phi(t, x):=x$ and $p=n)$. 
Proof of Theorem 4.9. The implication $(\mathrm{b}) \Rightarrow(\mathrm{c})$ is obvious and we prove implications (a) $\Rightarrow(\mathrm{b})$ and (c) $\Rightarrow(\mathrm{a})$. $(\mathbf{c}) \Rightarrow(\mathbf{a})$. Suppose that (1.3) admits an ORCLF. Without loss of generality, we may assume that the function $q \in \boldsymbol{E}$ involved in (4.9) is positive for all $t \geqslant 0$.

Furthermore define:

$$
\begin{gathered}
\Xi(t, \varphi, u):=\Psi(t, \varphi, u)-8 q(t), \quad(t, \varphi, u) \in \Re^{+} \times \Re^{p} \times U \\
\Xi(t, \varphi, u):=\Xi(0, \varphi, u), \quad(t, \varphi, u) \in(-1,0) \times \Re^{p} \times U .
\end{gathered}
$$

The definition of $\Xi$, given by (4.13a), (4.13b), guarantees that the function $\Xi:(-1,+\infty) \times \Re \Re^{p} \times U \rightarrow \Re \cup\{+\infty\}$ with $\Xi(t, 0,0)=-8 q(\max \{0, t\})$ for all $t>-1$ is such that, for each $u \in U$ the mapping $(t, \varphi) \rightarrow \Xi(t, \varphi, u)$ is upper semi-continuous. By virtue of (4.9) and upper semi-continuity of $\Xi$, it follows that for each $(t, \varphi) \in$ $(-1,+\infty) \times \Re^{p}$ there exist $u=u(t, \varphi) \in U$ and $\delta=\delta(t, \varphi) \in(0, t+1)$, such that

$$
\Xi(\tau, y, u(t, \varphi)) \leqslant 0, \forall(\tau, y) \in\left\{(\tau, y) \in(-1,+\infty) \times \Re^{p}:|\tau-t|+|y-\varphi|<\delta\right\}
$$

Using (4.14) and standard partition of unity arguments, we can determine sequences $\left\{\left(t_{i}, \varphi_{i}\right) \in(-1,+\infty) \times\right.$ $\left.\Re^{p}\right\}_{i=1}^{\infty},\left\{u_{i} \in U\right\}_{i=1}^{\infty},\left\{\delta_{i}\right\}_{i=1}^{\infty}$ with $\delta_{i}=\delta\left(t_{i}, \varphi_{i}\right) \in\left(0, t_{i}+1\right)$ associated with a sequence of open sets $\left\{\Omega_{i}\right\}_{i=1}^{\infty}$ with

$$
\Omega_{i} \subseteq\left\{(\tau, y) \in(-1,+\infty) \times \Re^{p}:\left|\tau-t_{i}\right|+\left|y-\varphi_{i}\right|<\delta_{i}\right\}
$$

forming a locally finite open covering of $(-1,+\infty) \times \Re^{p}$ and in such a way that:

$$
\Xi\left(\tau, y, u_{i}\right) \leqslant 0, \quad \forall(\tau, y) \in \Omega_{i}
$$

Also, a family of smooth functions $\left\{\theta_{i}\right\}_{i=1}^{\infty}$ with $\theta_{i}(t, \varphi) \geqslant 0$ for all $(t, x) \in(-1,+\infty) \times \Re^{p}$ can be determined with

$$
\begin{gathered}
\operatorname{supp} \theta_{i} \subseteq \Omega_{i} \\
\sum_{i=1}^{\infty} \theta_{i}(t, \varphi)=1, \quad \forall(t, \varphi) \in(-1,+\infty) \times \Re^{p} .
\end{gathered}
$$

Using exactly the same methodology as in the proof of implication $(d) \Rightarrow($ a) of Theorem 2.8 and the facts that $\Xi(t, 0,0)=-8 q(t)<0$ for all $t \geqslant 0$ and that the mapping $(t, \varphi) \rightarrow \Xi(t, \varphi, 0)$ is upper semi-continuous, we may establish the existence of a $C^{\infty}$ positive function $\eta: \Re^{+} \rightarrow(0,+\infty)$ with the following property:

$$
|\varphi| \leqslant 2 \eta(t) \Rightarrow \Xi(t, \varphi, 0) \leqslant 0
$$

Let $h \in C^{\infty}(\Re ;[0,1])$ be a smooth non-decreasing function with $h(s)=0$ for all $s \leqslant 0$ and $h(s)=1$ for all $s \geqslant 1$. We define for all $(t, x) \in \Re^{+} \times C^{0}\left([-r, 0] ; \Re^{n}\right)$ :

$$
k(t, x):=h\left(\frac{|\Phi(t, x)|^{2}-2 \eta^{2}(t)}{2 \eta^{2}(t)}\right) \sum_{i=1}^{\infty} \theta_{i}(t, \Phi(t, x)) u_{i}
$$

where $\Phi=\left(\Phi_{1}, \ldots, \Phi_{p}\right)^{\prime}: \Re^{+} \times C^{0}\left([-r, 0] ; \Re^{n}\right) \rightarrow \Re^{p}$ is the mapping involved in property (ii) of Definition 4.7. Clearly, $k$ as defined by (4.20) is a mapping satisfying the property that for every bounded $\Omega \subset$ $\Re^{+} \times C^{0}\left([-r, 0] ; \Re^{n}\right)$ it holds that $\sup \left\{\frac{|k(t, x)-k(t, y)|}{\|x-y\|_{r}}:(t, x) \in \Omega,(t, y) \in \Omega, x \neq y\right\}<+\infty$, with $k(t, 0)=0$ for all $t \geqslant 0$. Moreover, since $k(t, x)$ is defined as a (finite) convex combination of $u_{i} \in U$ and $0 \in U$, we have $k(t, x) \in U$ for all $(t, x) \in \Re^{+} \times C^{0}\left([-r, 0] ; \Re^{n}\right)$. 
Let $(t, x) \in \Re^{+} \times C^{0}\left([-r, 0] ; \Re^{n}\right)$ with $|\Phi(t, x)| \geqslant 2 \eta(t)$ and define $J(t, x)=\left\{j \in\{1,2, \ldots\} ; \theta_{j}(t, \Phi(t, x)) \neq 0\right\}$ (a finite set). Notice that by virtue of (4.10) and definition (4.20) we get:

$$
\begin{aligned}
\sup _{d \in D} V^{0}(t, x ; f(t, d, x, k(t, x))) & =\sup _{d \in D} V^{0}\left(t, x ; f\left(t, d, x, \sum_{j \in J(t, x)} \theta_{j}(t, \Phi(t, x)) u_{j}\right)\right) \\
& \leqslant-\rho(V(t, x))+\max _{j \in J(t, x)}\left\{\Psi\left(t, \Phi(t, x), u_{j}\right)\right\} .
\end{aligned}
$$

Notice that for each $j \in J(t, x)$ we obtain from (4.17) that $(t, \Phi(t, x)) \in \Omega_{j}$. Consequently, by virtue of (4.16) and definition (4.13a) we have that $\Psi\left(t, \Phi(t, x), u_{j}\right) \leqslant 8 q(t)$, for all $j \in J(t, x)$. Combining the previous inequality with inequality (4.21), we conclude that the following property holds for all $(t, x, d) \in \Re^{+} \times C^{0}\left([-r, 0] ; \Re^{n}\right) \times D$ with $|\Phi(t, x)| \geqslant 2 \eta(t)$ :

$$
V^{0}(t, x ; f(t, d, x, k(t, x))) \leqslant-\rho(V(t, x))+8 q(t) .
$$

Let $(t, x) \in \Re^{+} \times C^{0}\left([-r, 0] ; \Re^{n}\right)$ with $|\Phi(t, x)| \leqslant \sqrt{2} \eta(t)$. Notice that by virtue of definition (4.20) we get:

$$
\sup _{d \in D} V^{0}(t, x ; f(t, d, x, k(t, x)))=\sup _{d \in D} V^{0}(t, x ; f(t, d, x, 0)) .
$$

By virtue of (4.10), (4.19), (4.13a) and the above inequality we conclude that (4.22) holds as well for all $(t, x) \in \Re^{+} \times C^{0}\left([-r, 0] ; \Re^{n}\right)$ with $|\Phi(t, x)| \leqslant \sqrt{2} \eta(t)$. Finally, for the case $(t, x) \in \Re^{+} \times C^{0}\left([-r, 0] ; \Re^{n}\right)$ with $\sqrt{2} \eta(t)<|\Phi(t, x)|<2 \eta(t)$, let $J(t, x)=\left\{j \in\{1,2, \ldots\} ; \theta_{j}(t, \Phi(t, x)) \neq 0\right\}$ and notice that from (4.10) we get:

$$
\begin{aligned}
\sup _{d \in D} V^{0}(t, x ; f(t, d, x, k(t, x))) & =\sup _{d \in D} V^{0}\left(t, x ; f\left(t, d, x, h\left(\frac{|x|^{2}-2 \eta^{2}(t)}{2 \eta^{2}(t)}\right) \sum_{j \in J(t, x)} \theta_{j}(t, x) u_{j}\right)\right) \\
& \leqslant-\rho(V(t, x))+\max \left\{\Psi(t, \Phi(t, x), 0), \Psi\left(t, \Phi(t, x), u_{j}\right), j \in J(t, x)\right\} .
\end{aligned}
$$

Taking into account definition (4.13a) and (4.16), (4.17), (4.19), (4.23), we may conclude that (4.22) holds as well for all $(t, x) \in \Re^{+} \times C^{0}\left([-r, 0] ; \Re^{n}\right)$ with $\sqrt{2} \eta(t)<|\Phi(t, x)|<2 \eta(t)$. Consequently, (4.22) holds for all $(t, x) \in \Re^{+} \times C^{0}\left([-r, 0] ; \Re^{n}\right)$.

It follows from (4.22) and Proposition 4.6 that system (1.3) with $u=k\left(t, T_{r}(t) x\right)$ is RGAOS.

(a) $\Rightarrow(\mathbf{b})$. Since system (1.3) with $u=k\left(t, T_{r}(t) x\right)$ is RGAOS, and since for every bounded $\Omega \subset \Re^{+} \times$ $C^{0}\left([-r, 0] ; \Re^{n}\right)$ it holds that $\sup \left\{\frac{|k(t, x)-k(t, y)|}{\|x-y\|_{r}}:(t, x) \in \Omega,(t, y) \in \Omega, x \neq y\right\}<+\infty$, it follows that the closed-loop system (1.3) with $u=k\left(t, T_{r}(t) x\right)$ satisfies hypotheses (R1)-(R4). Moreover, since system (1.3) with $u=k\left(t, T_{r}(t) x\right)$ is RGAOS, it follows from Theorem 3.6 in [13] that there exists $\mu \in K^{+}$such that the following system

$$
\begin{aligned}
& \dot{x}(t)=f\left(t, d(t), T_{r}(t) x, k\left(t, T_{r}(t) x\right)\right) \\
& Y(t)=\|H(t, x)\|_{\mathcal{Y}}+\mu(t)\|x\|_{r} \\
& x(t) \in \Re^{n}, Y(t) \in \Re, d(t) \in D
\end{aligned}
$$

satisfies hypotheses (R1)-(R4) and is RGAOS. Notice that system (4.24) is the closed-loop system (1.3) with $u=k\left(t, T_{r}(t) x\right)$ and output defined by $Y(t)=\|H(t, x)\|_{\mathcal{Y}}+\mu(t)\|x\|_{r}$. It follows from Theorem 3.5 in [20] that there exist functions $a_{1}, a_{2} \in K_{\infty}, \beta \in K^{+}$and a mapping $V: \Re^{+} \times C^{0}\left([-r, 0] ; \Re^{n}\right) \rightarrow \Re^{+}$, which is almost Lipschitz on bounded sets, such that:

$$
\begin{gathered}
a_{1}\left(\|H(t, x)\|_{\mathcal{Y}}+\mu(t)\|x\|_{r}\right) \leqslant V(t, x) \leqslant a_{2}\left(\beta(t)\|x\|_{r}\right), \quad \forall(t, x) \in \Re^{+} \times C^{0}\left([-r, 0] ; \Re^{n}\right) \\
V^{0}(t, x ; f(t, d, x, k(t, x))) \leqslant-V(t, x), \quad \forall(t, x, d) \in \Re^{+} \times C^{0}\left([-r, 0] ; \Re^{n}\right) \times D .
\end{gathered}
$$


We next prove that $V$ is an ORCLF for (1.3). Obviously property (i) of Definition 4.7 is a consequence of inequalities (4.25). Define for all $(t, \varphi)=\left(t, \varphi_{1}, \varphi_{2}\right)^{\prime} \in \Re^{+} \times \Re \times \Re^{m}$ :

$$
\Psi\left(t, \varphi_{1}, \varphi_{2}, u\right):=L_{V}\left(t+\left|\varphi_{1}\right|\right) L_{U}\left(t+\left|\varphi_{1}\right|+2\left|\varphi_{2}\right|+\left|u-\operatorname{Pr}_{U}\left(\varphi_{2}\right)\right|\right)\left|u-\operatorname{Pr}_{U}\left(\varphi_{2}\right)\right|
$$

and for all $(t, x) \in \Re^{+} \times C^{0}\left([-r, 0] ; \Re^{n}\right)$ :

$$
\Phi(t, x):=\left[\begin{array}{c}
\|x\|_{r} \\
k(t, x)
\end{array}\right] \in \Re^{m+1}
$$

where $L_{U}: \Re^{+} \rightarrow \Re^{+}$is the non-decreasing continuous function involved in (4.2) and $L_{V}: \Re^{+} \rightarrow \Re^{+}$is the non-decreasing function involved in property $(\mathrm{P} 1)$ of Definition 4.5 . The reader should notice that $\Phi(t, 0)=0$ for all $t \geqslant 0$ and that for every bounded $\Omega \subset \Re^{+} \times C^{0}\left([-r, 0] ; \Re^{n}\right)$ it holds that

$$
\sup \left\{\frac{|\Phi(t, x)-\Phi(t, y)|}{\|x-y\|_{r}}:(t, x) \in \Omega,(t, y) \in \Omega, x \neq y\right\}<+\infty
$$

Without loss of generality we may assume that $L_{V}: \Re^{+} \rightarrow \Re^{+}$is continuous as well. Convexity of the set $U \subseteq \Re^{m}$ implies that the mapping $\Re^{m}$ э $\varphi_{2} \rightarrow \operatorname{Pr}_{U}\left(\varphi_{2}\right)$ is continuous and consequently that the mapping $\Re^{+} \times$ $\Re^{m+1} \ni\left(t, \varphi_{1}, \varphi_{2}\right) \rightarrow \Psi\left(t, \varphi_{1}, \varphi_{2}, u\right)$ is continuous for each fixed $u \in U$. Notice that for every $\varphi=\left(t, \varphi_{1}, \varphi_{2}\right)^{\prime} \in$ $\Re^{+} \times \Re \times \Re^{m}$ and every finite set $\left\{u_{1}, u_{2}, \ldots, u_{N}\right\} \subset U, \lambda_{i} \in[0,1](i=1, \ldots, N)$ with $\sum_{i=1}^{N} \lambda_{i}=1$, definition (4.27) in conjunction with the fact $\left|\sum_{i=1}^{N} \lambda_{i} u_{i}-\operatorname{Pr}_{U}\left(\varphi_{2}\right)\right| \leqslant \sum_{i=1}^{N} \lambda_{i}\left|u_{i}-\operatorname{Pr}_{U}\left(\varphi_{2}\right)\right| \leqslant \max _{i=1, \ldots, N}\left|u_{i}-\operatorname{Pr}_{U}\left(\varphi_{2}\right)\right|$ implies that:

$$
\Psi\left(t, \varphi_{1}, \varphi_{2}, \sum_{i=1}^{N} \lambda_{i} u_{i}\right) \leqslant \max _{i=1, \ldots, N} \Psi\left(t, \varphi_{1}, \varphi_{2}, u_{i}\right)
$$

By virtue of definitions (4.4), (4.5) and property (P1) of Definition 4.5 we get for all $(t, x, v, w) \in \Re^{+} \times$ $C^{0}\left([-r, 0] ; \Re^{n}\right) \times \Re^{n} \times \Re^{n}:$

$$
V^{0}(t, x ; v) \leqslant L_{V}\left(t+\|x\|_{r}\right)|v-w|+V^{0}(t, x ; w)
$$

Combining inequalities (4.2), (4.26) and (4.30) we obtain for all $(t, x, u) \in \Re^{+} \times C^{0}\left([-r, 0] ; \Re^{n}\right) \times U$ :

$$
\begin{aligned}
\sup _{d \in D} V^{0}(t, x ; f(t, d, x, u)) & \leqslant \sup _{d \in D} V^{0}(t, x ; f(t, d, x, k(t, x)))+L_{V}\left(t+\|x\|_{r}\right) \sup _{d \in D}|f(t, d, x, u)-f(t, d, x, k(t, x))| \\
& \leqslant-V(t, x)+L_{V}\left(t+\|x\|_{r}\right) L_{U}\left(t+\|x\|_{r}+|u|+|k(t, x)|\right)|u-k(t, x)| \\
& \leqslant-V(t, x)+L_{V}\left(t+\|x\|_{r}\right) L_{U}\left(t+\|x\|_{r}+|u-k(t, x)|+2|k(t, x)|\right)|u-k(t, x)|
\end{aligned}
$$

The above inequality in conjunction with (4.29) and definitions (4.27), (4.28) implies that inequality (4.10) with $\rho(s):=s$ holds. Moreover, by virtue of definition (4.27), for every $\left(t, \varphi_{1}, \varphi_{2}\right) \in \Re^{+} \times \Re \times \Re^{m}$ there exists $u \in U$ (namely $u=\operatorname{Pr}_{U}\left(\varphi_{2}\right)$ ) such that (4.11) holds with $q(t) \equiv 0$. Notice that $\left|\operatorname{Pr}_{U}\left(\varphi_{2}\right)\right| \leqslant 2\left|\varphi_{2}\right| \leqslant 2|\varphi|$, where $\varphi=\left(\varphi_{1}, \varphi_{2}\right) \in \Re^{m+1}$ and therefore the small control property holds with $a(s):=2 s$ and $\gamma(t) \equiv 1$.

The proof is complete. 
Proof of Theorem 4.10. (a) $\Rightarrow(\mathbf{b})$. Suppose that (1.3) admits an ORCLF which satisfies the small control property with $q(t) \equiv 0$. Without loss of generality we may assume that the mapping $\Phi=\left(\Phi_{1}, \ldots, \Phi_{p}\right)^{\prime}$ : $\Re^{+} \times C^{0}\left([-r, 0] ; \Re^{n}\right) \rightarrow \Re^{p}$ involved in property (ii) of Definition 4.7 satisfies $\Phi_{1}(t, x):=V(t, x)$. Define:

$$
\begin{gathered}
\Xi(t, \varphi, u):=\Psi(t, \varphi, u)-\frac{1}{2} \rho\left(c^{\prime} \varphi\right), \quad(t, \varphi, u) \in \Re^{+} \times \Re^{p} \times U \\
\Xi(t, \varphi, u):=\Xi(0, \varphi, u), \quad(t, \varphi, u) \in(-1,0) \times \Re^{p} \times U \\
c=(1,0, \ldots, 0)^{\prime} \in \Re^{p} .
\end{gathered}
$$

The definition of $\Xi$, given by (4.31), guarantees that the function $\Xi:(-1,+\infty) \times \Re^{p} \times U \rightarrow \Re \cup\{+\infty\}$ with $\Xi(t, 0,0)=0$ for all $t>-1$ is such that, for each $u \in U$ the mapping $(t, \varphi) \rightarrow \Xi(t, \varphi, u)$ is upper semi-continuous. Let $\Theta:=(-1,+\infty) \times\left\{\varphi \in \Re^{p}: c^{\prime} \varphi \neq 0\right\}$, which is an open set. By virtue of (4.11) with $q(t) \equiv 0$ and upper semi-continuity of $\Xi$, it follows that for each $(t, \varphi) \in \Theta$ there exist $u=u(t, \varphi) \in U$ with $|u| \leqslant a(\gamma(\max (0, t))|\varphi|)$ and $\delta=\delta(t, \varphi) \in(0, \min (1, t+1)), \delta(t, \varphi) \leqslant \frac{\left|c^{\prime} \varphi\right|}{2}$, such that

$$
\Xi(\tau, y, u(t, \varphi)) \leqslant 0, \forall(\tau, y) \in\{(\tau, y) \in \Theta:|\tau-t|+|y-\varphi|<\delta\} .
$$

Using (4.32) and standard partition of unity arguments, we can determine sequences $\left\{\left(t_{i}, \varphi_{i}\right) \in \Theta\right\}_{i=1}^{\infty},\left\{u_{i} \in\right.$ $U\}_{i=1}^{\infty},\left\{\delta_{i}\right\}_{i=1}^{\infty}$ with $\left|u_{i}\right| \leqslant a\left(\gamma\left(\max \left(0, t_{i}\right)\right)\left|\varphi_{i}\right|\right), \delta_{i}=\delta\left(t_{i}, \varphi_{i}\right) \in\left(0, \min \left(1, t_{i}+1\right)\right), \delta_{i}=\delta\left(t_{i}, \varphi_{i}\right) \leqslant \frac{\left|c^{\prime} \varphi_{i}\right|}{2}$ associated with a sequence of open sets $\left\{\Omega_{i}\right\}_{i=1}^{\infty}$ with

$$
\Omega_{i} \subseteq\left\{(\tau, y) \in \Theta:\left|\tau-t_{i}\right|+\left|y-\varphi_{i}\right|<\delta_{i}\right\}
$$

forming a locally finite open covering of $\Theta$ and in such a way that:

$$
\Xi\left(\tau, y, u_{i}\right) \leqslant 0, \quad \forall(\tau, y) \in \Omega_{i} .
$$

Also, a family of smooth functions $\left\{\theta_{i}\right\}_{i=1}^{\infty}$ with $\theta_{i}(t, \varphi) \geqslant 0$ for all $(t, \varphi) \in \Theta$ can be determined with

$$
\begin{gathered}
\operatorname{supp} \theta_{i} \subseteq \Omega_{i} \\
\sum_{i=1}^{\infty} \theta_{i}(t, \varphi)=1, \quad \forall(t, \varphi) \in \Theta .
\end{gathered}
$$

We define for all $(t, x) \in \Re^{+} \times C^{0}\left([-r, 0] ; \Re^{n}\right)$ :

$$
k(t, x):=\left(1-h\left(\frac{|\Phi(t, x)|^{2}-2 \eta^{2}(t)}{2 \eta^{2}(t)}\right)\right) K\left(t, \operatorname{Pr}_{Q(t)}(\Phi(t, x))\right)+h\left(\frac{|\Phi(t, x)|^{2}-2 \eta^{2}(t)}{2 \eta^{2}(t)}\right) \tilde{k}(t, x)
$$

where

$$
\begin{gathered}
\tilde{k}(t, x):=\sum_{i=1}^{\infty} \theta_{i}(t, \Phi(t, x)) u_{i} \text { for } t \geqslant 0, x \neq 0 \\
\tilde{k}(t, 0):=0 \text { for } t \geqslant 0
\end{gathered}
$$

where $h \in C^{\infty}(\Re ;[0,1])$ be a smooth non-decreasing function with $h(s)=0$ for all $s \leqslant 0$ and $h(s)=1$ for all $s \geqslant 1$ and $Q(t)=\left\{\varphi \in \Re^{p}:|\varphi| \leqslant 3 \eta(t)\right\}$. It follows from definition (4.37), (4.38) and that facts that the continuous mapping $\Phi$ is completely locally Lipschitz with respect to $x \in C^{0}\left([-r, 0] ; \Re^{n}\right)$ and that the continuous mapping 
$\varphi \rightarrow K(t, \varphi)$ is locally Lipschitz that $k$ is completely locally Lipschitz with respect to $x \in C^{0}\left([-r, 0] ; \Re^{n}\right)$ with $k(t, 0)=0$ for all $t \geqslant 0$. Moreover, it should be noticed that if the ORCLF $V$ and the function $\Psi$ involved in property (ii) of Definition 4.7 are time independent then the partition of unity arguments used above may be repeated on $\Theta:=\left\{\varphi \in \Re^{p}: c^{\prime} \varphi \neq 0\right\}$ instead of $\Theta:=(-1,+\infty) \times\left\{\varphi \in \Re^{p}: c^{\prime} \varphi \neq 0\right\}$. This implies that the constructed feedback is time invariant, provided that the mappings $\Phi=\left(\Phi_{1}, \ldots, \Phi_{p}\right)^{\prime}: \Re^{+} \times C^{0}\left([-r, 0] ; \Re^{n}\right) \rightarrow$ $\Re^{p}$ and $K: A \rightarrow U$ are time independent too.

Exploiting the properties of the mappings $\Xi:(-1,+\infty) \times \Re^{p} \times U \rightarrow \Re \cup\{+\infty\}, \Psi: \Re^{+} \times \Re^{p} \times U \rightarrow \Re \cup\{+\infty\}$, inequalities (4.12), (4.34), definitions (4.31a), (4.37) and the fact that the mapping $\Phi=\left(\Phi_{1}, \ldots, \Phi_{p}\right)^{\prime}: \Re^{+} \times$ $C^{0}\left([-r, 0] ; \Re^{n}\right) \rightarrow \Re^{p}$ involved in property (ii) of Definition 4.7 satisfies $\Phi_{1}(t, x):=V(t, x)$, we may establish (exactly in the same way as in the proof of Thm. 4.9) the following inequality:

$$
V^{0}(t, x ; f(t, d, x, k(t, x))) \leqslant-\frac{1}{2} \rho(V(t, x)), \quad \forall(t, x, d) \in \Re^{+} \times \Re^{n} \times D .
$$

The fact that system (1.3) with $u=k\left(t, T_{r}(t) x\right)$ is URGAOS follows directly from Proposition 4.6 and inequality (4.39).

(b) $\Rightarrow$ (a). Since for every bounded $\Omega \subset \Re^{+} \times C^{0}\left([-r, 0] ; \Re^{n}\right)$ it holds that

$$
\sup \left\{\frac{|k(t, x)-k(t, y)|}{\|x-y\|_{r}}:(t, x) \in \Omega,(t, y) \in \Omega, x \neq y\right\}<+\infty
$$

it follows that the closed-loop system (1.3) with $u=k\left(t, T_{r}(t) x\right)$ satisfies hypotheses (R1)-(R4). For each $\left(t_{0}, x_{0}, d\right) \in \Re^{+} \times C^{0}\left([-r, 0] ; \Re^{n}\right) \times M_{D}$, we denote the solution of (1.3) with $u=k\left(t, T_{r}(t) x\right)$, initial condition $T_{r}\left(t_{0}\right) x=x_{0}$ corresponding to $d \in M_{D}$ by $x\left(t, t_{0}, x_{0}, d\right)$.

Since system (1.3) with $u=k\left(t, T_{r}(t) x\right)$ is RFC, it follows from Lemma 3.5 in [13] that there exists $\tilde{\mu} \in K^{+}$, $a \in K_{\infty}$ such that the following inequality holds for all $\left(t_{0}, x_{0}, d\right) \in \Re^{+} \times C^{0}\left([-r, 0] ; \Re^{n}\right) \times M_{D}$ :

$$
\tilde{\mu}(t)\|x\|_{r} \leqslant a\left(\left\|x_{0}\right\|_{r}\right), \quad \forall t \geqslant t_{0}
$$

Since system (1.3) with $u=k\left(t, T_{r}(t) x\right)$ is URGAOS, it follows from (4.40) that the following system

$$
\begin{aligned}
& \dot{x}(t)=f\left(t, d(t), T_{r}(t) x, k\left(t, T_{r}(t) x\right)\right), \quad Y(t)=\|H(t, x)\|_{\mathcal{Y}}+\mu(t)\|x\|_{r} \\
& x(t) \in \Re^{n}, Y(t) \in \Re, d(t) \in D
\end{aligned}
$$

where $\mu(t):=\exp (-t) \tilde{\mu}(t)$ satisfies hypotheses $(\mathrm{R} 1)-(\mathrm{R} 4)$ and is URGAOS. Notice that system (4.41) is the closed-loop system (1.3) with $u=k\left(t, T_{r}(t) x\right)$ and output defined by $Y(t)=\|H(t, x)\|_{\mathcal{Y}}+\mu(t)\|x\|_{r}$. It follows from Theorem 3.6 in [20] that there exist functions $a_{1}, a_{2} \in K_{\infty}$ and a mapping $V: \Re^{+} \times C^{0}\left([-r, 0] ; \Re^{n}\right) \rightarrow \Re^{+}$, which is almost Lipschitz on bounded sets, such that:

$$
\begin{gathered}
a_{1}\left(\|H(t, x)\|_{\mathcal{Y}}+\mu(t)\|x\|_{r}\right) \leqslant V(t, x) \leqslant a_{2}\left(\|x\|_{r}\right), \quad \forall(t, x) \in \Re^{+} \times C^{0}\left([-r, 0] ; \Re^{n}\right) \\
V^{0}(t, x ; f(t, d, x, k(t, x))) \leqslant-V(t, x), \quad \forall(t, x, d) \in \Re^{+} \times C^{0}\left([-r, 0] ; \Re^{n}\right) \times D .
\end{gathered}
$$

The rest of proof is exactly the same as the proof of implication (a) $\Rightarrow$ (b) of Theorem 4.9. The only additional thing that should be noticed is that the mappings $\Phi=\left(\Phi_{1}, \ldots, \Phi_{p}\right)^{\prime}: \Re^{+} \times C^{0}\left([-r, 0] ; \Re^{n}\right) \rightarrow \Re^{p}, \eta \in K^{+}$ and $A$ э $(t, \varphi) \rightarrow K(t, \varphi) \in U$, may be selected so that (4.28) holds, $\eta(t) \equiv 1$ and $K(t, \varphi):=\operatorname{Pr}_{U}\left(\varphi_{2}\right)$ for all $(t, \varphi)=\left(t, \varphi_{1}, \varphi_{2}\right)^{\prime} \in \Re^{+} \times \Re \times \Re^{m}$.

The proof is complete. 


\section{Applications to tRiangular time-DELAY CONTROL SYSTEMS}

Our main result concerning triangular time-delay control systems of the form (1.4) is stated next. It must be compared to Theorem 5.1 in [7], which deals with the triangular finite-dimensional case.

Theorem 5.1. Consider system (1.4), where $r>0, D \subset \Re^{l}$ is a compact set, the mappings $f_{i}: \Re^{+} \times D \times$ $C^{0}\left([-r, 0] ; \Re^{i}\right) \rightarrow \Re, g_{i}: \Re^{+} \times D \times C^{0}\left([-r, 0] ; \Re^{i}\right) \rightarrow \Re(i=1, \ldots, n)$ are continuous with $f_{i}(t, d, 0)=0$ for all $(t, d) \in \Re^{+} \times D$ and each $g_{i}: \Re^{+} \times D \times C^{0}\left([-r, 0] ; \Re^{i}\right) \rightarrow \Re(i=1, \ldots, n)$ is completely locally Lipschitz with respect to $x \in C^{0}\left([-r, 0] ; \Re^{i}\right)$. Suppose that there exists a function $\varphi \in C^{\infty}\left(\Re^{+} ;(0,+\infty)\right)$ being non-decreasing, such that for every $i=1, \ldots, n$, it holds that:

$$
\frac{1}{\varphi\left(\|x\|_{r}\right)} \leqslant g_{i}(t, d, x) \leqslant \varphi\left(\|x\|_{r}\right), \quad \forall(t, x, d) \in \Re^{+} \times C^{0}\left([-r, 0] ; \Re^{i}\right) \times D .
$$

Moreover, suppose that for every $i=1, \ldots, n$, it holds that

$$
\sup \left\{\frac{\left|f_{i}(t, d, x)-f_{i}(t, d, y)\right|}{\|x-y\|_{r}}:(t, d) \in \Re^{+} \times D, x \in S, y \in S, x \neq y\right\}<+\infty,
$$

for every bounded $S \subset C^{0}\left([-r, 0] ; \Re^{i}\right)$.

Then for every $\sigma>0$ there exist functions $\mu_{i} \in C^{\infty}\left(\Re^{i} ;(0,+\infty)\right), k_{i} \in C^{\infty}\left(\Re^{i} ; \Re\right)(i=1, \ldots, n)$ with

$$
\begin{gathered}
k_{1}\left(\xi_{1}\right):=-\mu_{1}\left(\xi_{1}\right) \xi_{1} \\
k_{j}\left(\xi_{1}, \ldots, \xi_{j}\right):=-\mu_{j}\left(\xi_{1}, \ldots, \xi_{j}\right)\left(\xi_{j}-k_{j-1}\left(\xi_{1}, \ldots, \xi_{j-1}\right)\right), \quad j=2, \ldots, n
\end{gathered}
$$

such that the following functional:

$$
V(x):=\max _{\theta \in[-r, 0]} \exp (2 \sigma \theta)\left(x_{1}^{2}(\theta)+\sum_{j=2}^{n}\left|x_{j}(\theta)-k_{j-1}\left(x_{1}(\theta), \ldots, x_{j-1}(\theta)\right)\right|^{2}\right)
$$

is a State Robust Control Lyapunov Functional (SRCLF) for (1.4), which satisfies the "small-control" property. Moreover, the closed-loop system (1.4) with $u(t)=k_{n}(x(t))$ is URGAS. More specifically, the inequality $V^{0}(x ; v) \leqslant-2 \sigma V(x)$ holds for all $(t, x, d) \in \Re^{+} \times C^{0}\left([-r, 0] ; \Re^{n}\right) \times D$ with $v=\left(f_{1}\left(t, d, x_{1}\right)+g_{1}\left(t, d, x_{1}\right) x_{2}(0), \ldots\right.$, $\left.f_{n}(t, d, x)+g_{n}(t, d, x) k_{n}(x(0))\right)^{\prime} \in \Re^{n}$.

\section{Remark 5.2.}

(i) The reader should notice that the feedback law $u(t)=k_{n}(x(t))$ is delay-independent. The proof of Theorem 5.1 will show that the functions $\mu_{i} \in C^{\infty}\left(\Re^{i} ;(0,+\infty)\right)(i=1, \ldots, n)$ are obtained by a procedure similar to the backstepping procedure used for finite-dimensional triangular control systems. Consequently, as in the finite-dimensional case, the feedback design and the construction of the State Robust Control Lyapunov Functional proceed in parallel.

(ii) There are important differences between Theorem 5.1 and the results obtained by Jankovic in [10,11]. First of all, in $[10,11]$ Razumikhin functions are constructed in parallel with feedback design while Theorem 5.1 provides Lyapunov functionals. Moreover, the construction of the State Robust Control Lyapunov Functional can be automated (see the algorithmic procedure described below). Finally, we consider the general case of time-varying, uncertain systems. The reader should also notice that the results in [26,35] (which also provide Lyapunov functionals) are restricted to the disturbance free, autonomous case (1.4) with a single discrete delay and the mappings $g_{i}(i=1, \ldots, n)$ are free of delay, i.e., $g_{i}\left(t, d, T_{r}(t) x_{1}, \ldots, T_{r}(t) x_{i}\right)=g_{i}\left(x_{1}(t), \ldots, x_{i}(t)\right)$ for $i=1, \ldots, n$. 
The proof of Theorem 5.1 is based on the following lemma. Its proof is provided in the Appendix. The reader should notice that Lemma 5.3 in conjunction with definition (5.3) of the SRCLF for system (1.4) indicates one of the complications mentioned in the Introduction encountered in the study of infinite-dimensional systems: although the differential equations (1.4) are affine in the control input $u \in \Re$, the derivative $V^{0}(x ; v)$, where $v=\left(f_{1}\left(t, d, x_{1}\right)+g_{1}\left(t, d, x_{1}\right) x_{2}(0), \ldots, f_{n}(t, d, x)+g_{n}(t, d, x) u\right)^{\prime} \in \Re^{n}, x=\left(x_{1}, \ldots, x_{n}\right) \in C^{0}\left([-r, 0] ; \Re^{n}\right)$ is not affine in the control input $u \in \Re$.

Lemma 5.3. Let $Q \in C^{1}\left(\Re^{n} ; \Re^{+}\right), \sigma>0$ and consider the functional $V: C^{0}\left([-r, 0] ; \Re^{n}\right) \rightarrow \Re^{+}$defined by:

$$
V(x):=\max _{\theta \in[-r, 0]} \exp (2 \sigma \theta) Q(x(\theta))
$$

The functional $V: C^{0}\left([-r, 0] ; \Re^{n}\right) \rightarrow \Re^{+}$defined by (5.1), is Lipschitz on bounded sets of $C^{0}\left([-r, 0] ; \Re^{n}\right)$ and satisfies:

$$
\begin{aligned}
& V^{0}(x ; v) \leqslant-2 \sigma V(x) \text { for all }(x, v) \in C^{0}\left([-r, 0] ; \Re^{n}\right) \times \Re^{n} \text { with } Q(x(0))<V(x) \\
& V^{0}(x ; v) \leqslant \max \{-2 \sigma V(x), \nabla Q(x(0)) v\} \text { for all }(x, v) \in C^{0}\left([-r, 0] ; \Re^{n}\right) \times \Re^{n} \text { with } Q(x(0))=V(x) .
\end{aligned}
$$

We are now in a position to provide the proof of Theorem 5.1.

Proof of Theorem 5.1. Inequality (5.2) in conjunction with the fact that $f_{i}(t, d, 0)=0$ for all $(t, d) \in \Re^{+} \times D$ $(i=1, \ldots, n)$ implies the existence of a non-decreasing function $L \in C^{\infty}\left(\Re^{+} ;(0,+\infty)\right)$ such that for every $i=1, \ldots, n$, it holds:

$$
\left|f_{i}(t, d, x)\right| \leqslant L\left(\|x\|_{r}\right)\|x\|_{r}, \quad \forall(t, x, d) \in \Re^{+} \times C^{0}\left([-r, 0] ; \Re^{i}\right) \times D .
$$

Let $\sigma>0$ be a given number. We next define functions $\mu_{i} \in C^{\infty}\left(\Re^{i} ;(0,+\infty)\right), \gamma_{i} \in C^{\infty}\left(\Re^{+} ;(0,+\infty)\right)$, $b_{i} \in C^{\infty}\left(\Re^{+} ;(0,+\infty)\right)(i=1, \ldots, n)$ using the following algorithm.

\section{Algorithm.}

Step $i=1$. We define:

$$
\mu_{1}\left(\xi_{1}\right):=\frac{\gamma_{1}\left(1+\xi_{1}^{2}\right)+n \sigma}{b_{1}\left(1+\xi_{1}^{2}\right)}
$$

where

$$
\begin{gathered}
\gamma_{1}(s):=\exp (\sigma r) L(s \exp (\sigma r))+\varphi(s \exp (\sigma r)) \\
b_{1}(s):=\frac{1}{\varphi(s \exp (\sigma r))} .
\end{gathered}
$$

Step $i \geqslant 2$. Based on the knowledge of the functions $\mu_{j} \in C^{\infty}\left(\Re^{j} ;(0,+\infty)\right)(j=1, \ldots, i-1)$ from previous steps we define the function $\mu_{i} \in C^{\infty}\left(\Re^{i} ;(0,+\infty)\right)$. First define:

$$
\begin{gathered}
k_{0} \equiv 0, \quad k_{1}\left(\xi_{1}\right):=-\mu_{1}\left(\xi_{1}\right) \xi_{1} \\
k_{j}\left(\xi_{1}, \ldots, \xi_{j}\right):=-\mu_{j}\left(\xi_{1}, \ldots, \xi_{j}\right)\left(\xi_{j}-k_{j-1}\left(\xi_{1}, \ldots, \xi_{j-1}\right)\right), \quad j=2, \ldots, i-1 \\
\gamma_{j}(s):=\exp (\sigma r) L\left(s \exp (\sigma r) B_{j}(s \exp (\sigma r))\right) B_{j}(s \exp (\sigma r))+\varphi\left(s \exp (\sigma r) B_{j}(s \exp (\sigma r))\right), \quad j=1, \ldots, i \\
b_{j}(s):=\frac{1}{\varphi\left(s \exp (\sigma r) B_{j}(s \exp (\sigma r))\right)}, \quad j=1, \ldots, i
\end{gathered}
$$


where $B_{j} \in C^{\infty}\left(\Re^{+} ;(0,+\infty)\right)(j=1, \ldots, i)$ are non-decreasing functions that satisfy:

$B_{1}(s):=1, \quad B_{j}(s) \geqslant \max \left\{1+\sum_{l=1}^{j-1} \mu_{l}\left(\xi_{1}, \ldots, \xi_{l}\right): \max _{l=1, \ldots, j}\left|\xi_{l}-k_{l-1}\left(\xi_{1}, \ldots, \xi_{l-1}\right)\right| \leqslant s\right\}$, for all $s \geqslant 0$ and $j \geqslant 2$.

Let $\rho_{j} \in C^{\infty}\left(\Re^{+} ;(0,+\infty)\right)(j=1, \ldots, i)$ and $\delta_{j} \in C^{\infty}\left(\Re^{j} ;(0,+\infty)\right)(j=0, \ldots, i-1)$ be functions such that the following inequalities hold:

$$
\begin{gathered}
b_{j}\left(s^{\prime}\right)-b_{j}(s)+s \gamma_{j}(s)-s^{\prime} \gamma_{j}\left(s^{\prime}\right) \leqslant\left(s-s^{\prime}\right) \rho_{j}(s), \quad \forall s \geqslant s^{\prime} \geqslant 0 \\
\delta_{j}\left(\xi_{1}, \ldots, \xi_{j}\right) \geqslant\left|\nabla k_{j}\left(\xi_{1}, \ldots, \xi_{j}\right)\right|\left(1+\mu_{1}\left(\xi_{1}\right)+\ldots+\mu_{j}\left(\xi_{1}, \ldots, \xi_{j}\right)\right), \quad \forall\left(\xi_{1}, \ldots, \xi_{j}\right) \in \Re^{j} .
\end{gathered}
$$

Define:

$$
\mu_{i}\left(\xi_{1}, \ldots, \xi_{i}\right):=b_{i}^{-1}(p)\left[(n+1-i) \sigma+\frac{i-1}{4 \sigma} a^{2}\left(p, \xi_{1}, \ldots, \xi_{j-1}\right)+\gamma_{i}(p)+c_{i-1}(p) \delta_{i-1}\left(\xi_{1}, \ldots, \xi_{j-1}\right)\right]
$$

where

$$
\begin{gathered}
p:=\frac{i}{2}+\frac{1}{2} \sum_{j=1}^{i}\left|\xi_{j}-k_{j-1}\left(\xi_{1}, \ldots, \xi_{j-1}\right)\right|^{2}, \quad c_{0} \equiv 0, \quad c_{j}(s):=\sum_{k=1}^{j} \gamma_{k}(s) \text { for } j=1, \ldots, i \\
a\left(s, \xi_{1}, \ldots, \xi_{i-1}\right):=c_{i-1}(s) \delta_{i-1}\left(\xi_{1}, \ldots, \xi_{i-1}\right)+c_{i}(s)+\left(1+\sum_{j=1}^{i-1}\left(s \mu_{j}\left(\xi_{1}, \ldots, \xi_{j}\right)+\delta_{j-1}\left(\xi_{1}, \ldots, \xi_{j-1}\right)\right)\right) \sum_{k=1}^{i-1} \rho_{k}(s) .
\end{gathered}
$$

It should be noticed that in every step $i \geqslant 2$ of the above algorithm we only need to compute the functions $\gamma_{i}(s), b_{i}(s), B_{i}(s), \rho_{i-1}(s), \delta_{i-1}\left(\xi_{1}, \ldots, \xi_{i-1}\right)$ and $\mu_{i}\left(\xi_{1}, \ldots, \xi_{i}\right)$ (the functions $\gamma_{j}(s), b_{j}(s), B_{j}(s), \rho_{j-1}(s)$, $\delta_{j-1}\left(\xi_{1}, \ldots, \xi_{j-1}\right)$ and $\mu_{j}\left(\xi_{1}, \ldots, \xi_{j}\right)$ for $j=1, \ldots, i-1$ have been computed in the previous steps). It can be shown by induction (see Appendix) that the following claim holds:

Claim. The following inequality holds for all $\left(\xi_{1}, \ldots, \xi_{i}\right)^{\prime} \in \Re^{i}$ :

$$
\begin{aligned}
-\sum_{j=1}^{i}\left(\xi_{j}-\right. & \left.k_{j-1}\left(\xi_{1}, \ldots, \xi_{j-1}\right)\right)^{2} b_{j}(s) \mu_{j}\left(\xi_{1}, \ldots, \xi_{j}\right) \\
& +s \sum_{j=1}^{i}\left|\xi_{j}-k_{j-1}\left(\xi_{1}, \ldots, \xi_{j-1}\right)\right| \gamma_{j}(s)+s \sum_{j=1}^{i}\left|\xi_{j}-k_{j-1}\left(\xi_{1}, \ldots, \xi_{j-1}\right)\right| c_{j-1}(s) \delta_{j-1}\left(\xi_{1}, \ldots, \xi_{j-1}\right) \\
& \leqslant-(n+1-i) \sigma \sum_{j=1}^{i}\left|\xi_{j}-k_{j-1}\left(\xi_{1}, \ldots, \xi_{j-1}\right)\right|^{2}
\end{aligned}
$$

where

$$
s=\sum_{j=1}^{i}\left|\xi_{j}-k_{j-1}\left(\xi_{1}, \ldots, \xi_{j-1}\right)\right|, \quad c_{0} \equiv 0, \quad c_{j}(s):=\sum_{k=1}^{j} \gamma_{k}(s) \text { for } j=1, \ldots, i .
$$


By virtue of Lemma 5.3 it follows that the functional $V$ defined by (5.4) satisfies:

$V^{0}(x ; v) \leqslant-2 \sigma V(x)$

for all $(t, x, u, d) \in \Re^{+} \times C^{0}\left([-r, 0] ; \Re^{n}\right) \times \Re \times D$ with $V(x)>x_{1}^{2}(0)+\sum_{j=2}^{n}\left|x_{j}(0)-k_{j-1}\left(x_{1}(0), \ldots, x_{j-1}(0)\right)\right|^{2}$ and $v=\left(f_{1}\left(t, d, x_{1}\right)+g_{1}\left(t, d, x_{1}\right) x_{2}(0), \ldots, f_{n}(t, d, x)+g_{n}(t, d, x) u\right)^{\prime} \in \Re^{n}$

$V^{0}(x ; v) \leqslant \max \{-2 \sigma V(x), 2 A(t, d, x, u)\}$

for all $(t, x, u, d) \in \Re^{+} \times C^{0}\left([-r, 0] ; \Re^{n}\right) \times \Re \times D$ with $V(x)=x_{1}^{2}(0)+\sum_{j=2}^{n}\left|x_{j}(0)-k_{j-1}\left(x_{1}(0), \ldots, x_{j-1}(0)\right)\right|^{2}$ and $v=\left(f_{1}\left(t, d, x_{1}\right)+g_{1}\left(t, d, x_{1}\right) x_{2}(0), \ldots, f_{n}(t, d, x)+g_{n}(t, d, x) u\right)^{\prime} \in \Re^{n}$

where

$$
\begin{array}{r}
A(t, d, x, u):=\sum_{j=1}^{n-1}\left(x_{j}(0)-k_{j-1}\left(x_{1}(0), \ldots, x_{j-1}(0)\right)\right)\left(f_{j}\left(t, d, x_{1}, \ldots, x_{j}\right)+g_{j}\left(t, d, x_{1}, \ldots, x_{j}\right) x_{j+1}(0)\right)-\sum_{j=2}^{n-1}\left(x_{j}(0)\right. \\
\left.-k_{j-1}\left(x_{1}(0), \ldots, x_{j-1}(0)\right)\right)\left(\sum_{l=1}^{j-1} \frac{\partial k_{j-1}}{\partial \xi_{l}}\left(x_{1}(0), \ldots, x_{j-1}(0)\right)\left(f_{l}\left(t, d, x_{1}, \ldots, x_{l}\right)+g_{l}\left(t, d, x_{1}, \ldots, x_{l}\right) x_{l+1}(0)\right)\right) \\
\quad+\left(x_{n}(0)-k_{n-1}\left(x_{1}(0), \ldots, x_{n-1}(0)\right)\right)\left(f_{n}(t, d, x)+g_{n}(t, d, x) u\right) \\
-\left(x_{n}(0)-k_{n-1}\left(x_{1}(0), \ldots, x_{n-1}(0)\right)\right) \sum_{l=1}^{n-1} \frac{\partial k_{n-1}}{\partial \xi_{l}}\left(x_{1}(0), \ldots, x_{n-1}(0)\right)\left(f_{l}\left(t, d, x_{1}, \ldots, x_{l}\right)+g_{l}\left(t, d, x_{1}, \ldots, x_{l}\right) x_{l+1}(0)\right) .
\end{array}
$$

By virtue of the previous claim it can be shown (see Appendix) that for all $(t, x, d) \in \Re^{+} \times C^{0}\left([-r, 0] ; \Re^{n}\right) \times D$ with $u=k_{n}\left(x_{1}(0), \ldots, x_{n}(0)\right)$ and $V(x)=x_{1}^{2}(0)+\sum_{j=2}^{n}\left|x_{j}(0)-k_{j-1}\left(x_{1}(0), \ldots, x_{j-1}(0)\right)\right|^{2}$ the following inequalities hold:

$$
\begin{gathered}
A(t, d, x, u) \leqslant-\sigma \sum_{j=1}^{n}\left|x_{j}(0)-k_{j-1}\left(x_{1}(0), \ldots, x_{j-1}(0)\right)\right|^{2} \\
\max _{\theta \in[-r, 0]} \sum_{j=1}^{i}\left|x_{j}(\theta)\right| \leqslant s B_{i}(s), \text { with } s=\exp (\sigma r) \sum_{j=1}^{i}\left|x_{j}(0)-k_{j-1}\left(x_{1}(0), \ldots, x_{j-1}(0)\right)\right| .
\end{gathered}
$$

Consequently, by virtue of (5.17), (5.18) and (5.20) we obtain:

$V^{0}(x ; v) \leqslant-2 \sigma V(x)$, for all $(t, x, d) \in \Re^{+} \times C^{0}\left([-r, 0] ; \Re^{n}\right) \times D$ with

$$
v=\left(f_{1}\left(t, d, x_{1}\right)+g_{1}\left(t, d, x_{1}\right) x_{2}(0), \ldots, f_{n}(t, d, x)+g_{n}(t, d, x) k_{n}(x(0))\right)^{\prime} \in \Re^{n} .
$$

The reader should notice that there exist functions $a_{1}, a_{2} \in K_{\infty}$ such that

$$
a_{1}(|\xi|) \leqslant \xi_{1}^{2}+\sum_{j=2}^{n}\left|\xi_{j}-k_{j-1}\left(\xi_{1}, \ldots, \xi_{j-1}\right)\right|^{2} \leqslant a_{2}(|\xi|), \quad \forall \xi=\left(\xi_{1}, \ldots, \xi_{n}\right)^{\prime} \in \Re^{n}
$$


Consequently, definition (5.4) in conjunction with (5.23) implies

$$
\exp (-2 \sigma r) a_{1}\left(\|x\|_{r}\right) \leqslant V(x) \leqslant a_{2}\left(\|x\|_{r}\right), \quad \forall x \in C^{0}\left([-r, 0] ; \Re^{n}\right) .
$$

It follows from inequalities (5.22), (5.24) and Proposition 4.6 that the closed-loop system (1.4) with $u(t)=$ $k_{n}(x(t))$ is URGAS.

Finally, we show that $V$ as defined by (5.4) is a SRCLF, which satisfies the "small-control" property. Clearly, definition (5.19) in conjunction with (5.20), implies for all $(t, x, u, d) \in \Re^{+} \times C^{0}\left([-r, 0] ; \Re^{n}\right) \times \Re \times D$ with $V(x)=x_{1}^{2}(0)+\sum_{j=2}^{n}\left|x_{j}(0)-k_{j-1}\left(x_{1}(0), \ldots, x_{j-1}(0)\right)\right|^{2}:$

$$
\begin{aligned}
A(t, d, x, u) \leqslant-\sigma\left(x_{1}^{2}(0)+\right. & \left.\sum_{j=2}^{n}\left|x_{j}(0)-k_{j-1}\left(x_{1}(0), \ldots, x_{j-1}(0)\right)\right|^{2}\right) \\
& +\left|x_{n}(0)-k_{n-1}\left(x_{1}(0), \ldots, x_{n-1}(0)\right)\right|\left|g_{n}(t, d, x)\right|\left|u-k_{n}\left(x_{1}(0), \ldots, x_{n}(0)\right)\right| .
\end{aligned}
$$

By virtue of (5.1), (5.11a), (5.17), (5.18), (5.21) and (5.25) we obtain:

$$
\begin{gathered}
V^{0}(x ; v) \leqslant-2 \sigma V(x)+2\left|x_{n}(0)-k_{n-1}\left(x_{1}(0), \ldots, x_{n-1}(0)\right)\right| \gamma_{n}\left(\left|x_{1}(0)\right|+\sum_{j=2}^{n}\left|x_{j}(0)-k_{j-1}\left(x_{1}(0), \ldots, x_{j-1}(0)\right)\right|\right) \\
\times\left|u-k_{n}\left(x_{1}(0), \ldots, x_{n}(0)\right)\right| \text { for all }(t, x, u, d) \in \Re^{+} \times C^{0}\left([-r, 0] ; \Re^{n}\right) \times \Re \times D \text { with } \\
v=\left(f_{1}\left(t, d, x_{1}\right)+g_{1}\left(t, d, x_{1}\right) x_{2}(0), \ldots, f_{n}(t, d, x)+g_{n}(t, d, x) u\right)^{\prime} \in \Re^{n} .
\end{gathered}
$$

Define:

$$
\Phi(t, x):=x(0), \quad \rho(w):=2 \sigma w \text { and }
$$

$$
\Psi(t, z, u):=2\left|z_{n}-k_{n-1}\left(z_{1}, \ldots, z_{n-1}\right)\right| \gamma_{n}\left(\left|z_{1}\right|+\sum_{j=2}^{n}\left|z_{j}-k_{j-1}\left(z_{1}, \ldots, z_{j-1}\right)\right|\right)\left|u-k_{n}\left(z_{1}, \ldots, z_{n}\right)\right| .
$$

The above definitions in conjunction with inequalities (5.24) and (5.26) guarantee that inequalities (4.7), (4.10) and (4.11) hold (with $q(t) \equiv 0$ ), for $V$ as defined by (5.4). Consequently, $V$ as defined by (5.4) is a SRCLF, which satisfies the "small-control" property. The proof is complete.

Example 5.4. Consider the control system:

$$
\begin{aligned}
\dot{x}_{1}(t)= & d_{1}(t) \int_{t-r}^{t} x_{1}^{2}(\theta) \mathrm{d} \theta+x_{2}(t) \\
\dot{x}_{2}(t)= & d_{2}(t)\left\|T_{r}(t) x_{2}\right\|_{r}+u(t) \\
& \left(x_{1}(t), x_{2}(t)\right) \in \Re^{2},\left(d_{1}(t), d_{2}(t)\right) \in[-1,1]^{2}, u(t) \in \Re .
\end{aligned}
$$

Clearly, system (5.27) is a control system described by RFDEs, which satisfies the hypotheses of Theorem 5.1. More specifically, inequality (5.1) holds with $\varphi \equiv 1$. In order to design a delay free stabilizing feedback for (5.27) we follow the algorithm in the proof of Theorem 5.1. Notice that inequality (5.7) holds with $L(w)=1+r w$. Let $\sigma>0$ be given.

Step $i=1$. We define:

$$
\begin{gathered}
\mu_{1}\left(\xi_{1}\right):=\exp (\sigma r)\left(1+r\left(1+\xi_{1}^{2}\right) \exp (\sigma r)\right)+1+2 \sigma \\
\gamma_{1}(s):=\exp (\sigma r)(1+r s \exp (\sigma r))+1, \quad b_{1}(s) \equiv 1 .
\end{gathered}
$$


Step $i=2$. We define:

$$
\begin{gathered}
k_{1}\left(\xi_{1}\right):=-\left(\exp (\sigma r)\left(1+r\left(1+\xi_{1}^{2}\right) \exp (\sigma r)\right)+1+2 \sigma\right) \xi_{1} \\
\delta_{1}\left(\xi_{1}\right):=\left(\exp (\sigma r)\left(1+r\left(1+3 \xi_{1}^{2}\right) \exp (\sigma r)\right)+1+2 \sigma\right)\left(\exp (\sigma r)\left(1+r\left(1+\xi_{1}^{2}\right) \exp (\sigma r)\right)+2+2 \sigma\right) \\
B_{2}(s):=\exp (\sigma r)\left(1+r\left(1+s^{2}\right) \exp (\sigma r)\right)+2+2 \sigma \\
\gamma_{2}(s):=1+\exp (\sigma r)\left(1+s r \exp (\sigma r) B_{2}(\exp (\sigma r) s)\right) B_{2}(\exp (\sigma r) s), \quad b_{2}(s) \equiv 1 \\
\rho_{1}(s):=\exp (\sigma r)(1+2 r s \exp (\sigma r))+1 \\
a\left(s, \xi_{1}\right):=\gamma_{1}(s) \delta_{1}\left(\xi_{1}\right)+\gamma_{1}(s)+\gamma_{2}(s)+\left(1+s \mu_{1}\left(\xi_{1}\right)\right) \rho_{1}(s)
\end{gathered}
$$

and

$$
\mu_{2}\left(\xi_{1}, \xi_{2}\right):=\sigma+\frac{1}{4 \sigma} a^{2}\left(p, \xi_{1}\right)+\gamma_{2}(p)+\gamma_{1}(p) \delta_{1}\left(\xi_{1}\right)
$$

where

$$
p:=1+\frac{1}{2} \xi_{1}^{2}+\frac{1}{2}\left|\xi_{2}+\left(\exp (\sigma r)\left(1+r\left(1+\xi_{1}^{2}\right) \exp (\sigma r)\right)+1+2 \sigma\right) \xi_{1}\right|^{2} .
$$

The stabilizing feedback law is given by:

$$
u(t)=-\mu_{2}\left(x_{1}(t), x_{2}(t)\right)\left(x_{2}(t)+\left(\exp (\sigma r)\left(1+r\left(1+x_{1}^{2}(t)\right) \exp (\sigma r)\right)+1+2 \sigma\right) x_{1}(t)\right) .
$$

By virtue of Theorem 5.1, the functional

$$
V(x):=\max _{\theta \in[-r, 0]} \exp (2 \sigma \theta)\left(x_{1}^{2}(\theta)+\left|x_{2}(\theta)+\left(\exp (\sigma r)\left(1+r\left(1+x_{1}^{2}(\theta)\right) \exp (\sigma r)\right)+1+2 \sigma\right) x_{1}(\theta)\right|^{2}\right)
$$

is a SRCLF which satisfies the small-control property and system (5.27) with (5.38) is URGAS.

\section{Conclusions}

In the present work we have shown how the well-known "Control Lyapunov Function (CLF)" methodology can be generalized to a broader class of nonlinear time-varying systems with both disturbance and control inputs, which include infinite-dimensional control systems described by retarded functional differential equations (RFDEs). Necessary and sufficient conditions for the existence of stabilizing feedback are developed for the non-affine uncertain finite-dimensional case (1.1). Moreover, sufficient conditions, which guarantee that a given function is an Output Robust Control Lyapunov function for (1.1) are given. The case of uncertain control systems described by RFDEs of the form (1.3) is studied and special results are developed for the triangular case (1.4) of control systems described by RFDEs. It is shown that the construction of a stabilizing feedback law for (1.4) proceeds in parallel with the construction of a State Robust Control Lyapunov Functional. Moreover, sufficient conditions for the existence and design of a delay-free stabilizing feedback law are given. It is our belief that the present work can be used as a starting point for the discovery of necessary and sufficient Lyapunov-like conditions for the existence of stabilizing feedback for a wide class of infinite-dimensional control systems. 
Having extended the "Artstein-Sontag" methodology, we are now in a position to compare it with the "CoronRosier" methodology:

- the "Artstein-Sontag" methodology allows the use of Lipschitz RCLFs while the "Coron-Rosier" methodology requires continuously differentiable RCLFs; this is exactly the reason why the "Artstein-Sontag" approach can be extended to infinite-dimensional systems while the "Coron-Rosier" approach faces important difficulties with infinite-dimensional systems;

- the "Artstein-Sontag" methodology can provide time-independent feedback laws while the "CoronRosier" methodology always gives time-varying feedback laws;

- the "Coron-Rosier" approach does not require convexity assumptions; on the other hand the "ArtsteinSontag" approach requires that the control set is convex and demands additional properties for the RCLF.

Future research can give further extensions for both methodologies so that some of the above difficulties can be overcome.

\section{Appendix}

Proof of Proposition 2.5. Let $x:\left[t_{0}, t_{\max }\right) \rightarrow \Re^{n}$ be the unique solution of $(2.1)$ with initial condition $x\left(t_{0}\right)=$ $x_{0} \in \Re^{n}$ corresponding to certain $d \in M_{D}$, where $t_{\max } \in\left(t_{0},+\infty\right]$ is the maximal existence time of the solution. Notice that the mapping $\left[t_{0}, t_{\max }\right)$ э $t \rightarrow V(t, x(t)) \in \Re^{+}$is locally Lipschitz. By virtue of Lemma 2.4 and inequality (2.7) it follows that

$$
\frac{\mathrm{d}}{\mathrm{d} t}(V(t, x(t))) \leqslant-\rho(V(t, x(t)))+q(t), \text { for all } t \in\left[t_{0}, t_{\max }\right) \backslash N
$$

where $N \subset\left[t_{0}, t_{\max }\right)$ is a set of zero Lebesgue measure.

Case 1. Hypothesis (Q2) holds.

Lemma 4.4 in [22] and inequality (A1) show that there exists $\sigma \in K L$ such that

$$
V(t, x(t)) \leqslant \sigma\left(V\left(t_{0}, x_{0}\right), t-t_{0}\right), \text { for all } t \in\left[t_{0}, t_{\max }\right) .
$$

Inequality (A2) in conjunction with inequality (2.6) gives:

$$
\mu(t)|x(t)| \leqslant a_{1}^{-1}\left(\sigma\left(a_{2}\left(\left|x_{0}\right|\right), 0\right)\right) \text {, for all } t \in\left[t_{0}, t_{\max }\right) .
$$

Inequality (A3) and a standard contradiction argument show that (2.1) is RFC and that $t_{\max }=+\infty$. The fact that (2.1) is URGAOS follows from (A2) in conjunction with inequality (2.6). Particularly, we obtain:

$$
|H(t, x(t))| \leqslant a_{1}^{-1}\left(\sigma\left(a_{2}\left(\left|x_{0}\right|\right), t-t_{0}\right)\right), \text { for all } t \geqslant t_{0} .
$$

Estimate (A4) implies that (2.1) is URGAOS.

Case 2. Hypotheses (Q1), (Q3) hold.

Lemma 3.2 in [18] and inequality (A1) show that there exists $\sigma \in K L$ such that

$$
V(t, x(t)) \leqslant \sigma\left(V\left(t_{0}, x_{0}\right)+R, t-t_{0}\right), \text { for all } t \in\left[t_{0}, t_{\max }\right)
$$

where $R:=\int_{0}^{+\infty} q(s) \mathrm{d} s$. Inequality (A5) in conjunction with inequality (2.6) gives:

$$
\mu(t)|x(t)| \leqslant a_{1}^{-1}\left(\sigma\left(a_{2}\left(\beta\left(t_{0}\right)\left|x_{0}\right|\right)+R, 0\right)\right) \text {, for all } t \in\left[t_{0}, t_{\max }\right) .
$$


Inequality (A6) and a standard contradiction argument show that (2.1) is RFC and that $t_{\max }=+\infty$. The fact that (2.1) is RGAOS follows from (A6) in conjunction with inequality (2.6). Particularly, we obtain:

$$
|H(t, x(t))| \leqslant a_{1}^{-1}\left(\sigma\left(a_{2}\left(\beta\left(t_{0}\right)\left|x_{0}\right|\right)+R, t-t_{0}\right)\right) \text {, for all } t \geqslant t_{0} .
$$

Estimate (A.7) shows that property P2 (Uniform Output Attractivity on compact sets of initial data) holds of Definition 2.2 holds. Lemma 3.5 in [14] implies that (2.1) is RGAOS.

The proof is complete.

Proof of Proposition 4.6. Consider a solution $x(t)$ of (4.3) under hypotheses (R1)-(R4) corresponding to arbitrary $d \in M_{D}$ with initial condition $T_{r}\left(t_{0}\right) x=x_{0} \in C^{1}\left([-r, 0] ; \Re^{n}\right)$. By virtue of Lemma 2.5 in [20], for every $T \in\left(t_{0}, t_{\max }\right)$, the mapping $\left[t_{0}, T\right] \ni t \rightarrow V\left(t, T_{r}(t) x\right)$ is absolutely continuous. It follows from (4.8) and Lemma 4.4 that

$$
\frac{\mathrm{d}}{\mathrm{d} t}\left(V\left(t, T_{r}(t) x\right)\right) \leqslant-\rho\left(V\left(t, T_{r}(t) x\right)\right)+q(t) \text { a.e. on }\left[t_{0}, t_{\max }\right) .
$$

The previous differential inequality in conjunction with Lemma 3.2 in [18] shows that there exists $\sigma \in K L$ such that

$$
V\left(t, T_{r}(t) x\right) \leqslant \sigma\left(V\left(t_{0}, x_{0}\right)+R, t-t_{0}\right) \text { for all } t \in\left[t_{0}, t_{\max }\right)
$$

where $R:=\int_{0}^{+\infty} q(s) \mathrm{d} s$. Using (4.7), (A9) and a standard contradiction argument we may show that $t_{\max }=+\infty$. It follows from Lemma 2.6 in [20] that the solution $x(t)$ of (4.3) under hypotheses (R1)-(R4) corresponding to arbitrary $d \in M_{D}$ with arbitrary initial condition $T_{r}\left(t_{0}\right) x=x_{0} \in C^{0}\left([-r, 0]\right.$; $\left.\Re^{n}\right)$ satisfies (A9) for all $t \geqslant t_{0}$. Moreover, by virtue of (A9) and (4.7) we may establish that system (4.3) under hypotheses (R1)-(R4) is RFC. Notice that inequality (A9) in conjunction with (4.7) provide the estimate $\left\|H\left(t, T_{r}(t) x\right)\right\|_{\mathcal{Y}} \leqslant$ $a_{1}^{-1}\left(\sigma\left(a_{2}\left(\beta\left(t_{0}\right)\left\|x_{0}\right\|_{r}\right)+R, t-t_{0}\right)\right)$ for all $t \geqslant t_{0}$, which establishes the fact that property P2 (Uniform Output Attractivity on bounded sets of initial data) holds for system (4.3). It follows from Lemma 3.3 in [13] that system (4.3) is RGAOS. Furthermore, if $\beta(t) \equiv 1$ and $q(t) \equiv 0$, then inequality (A9) in conjunction with (4.7) provide the estimate $\left\|H\left(t, T_{r}(t) x\right)\right\|_{\mathcal{Y}} \leqslant a_{1}^{-1}\left(\sigma\left(a_{2}\left(\left\|x_{0}\right\|_{r}\right), t-t_{0}\right)\right)$ for all $t \geqslant t_{0}$, which establishes that system (4.3) is URGAOS.

The proof is complete.

Proof of Lemma 5.3. The fact that the functional $V$ as defined by (5.5) is Lipschitz on bounded sets of $C^{0}\left([-r, 0] ; \Re^{n}\right)$ is a direct consequence of the fact that $Q \in C^{1}\left(\Re^{n} ; \Re^{+}\right)$(details are left to the reader). Consequently, as noticed in Section 4.II, we obtain the following simplification for the derivative $V^{0}(x ; v)$ defined by $(4.5)$ for all $(x, v) \in C^{0}\left([-r, 0] ; \Re^{n}\right) \times \Re^{n}$ :

$$
V^{0}(x ; v)=\limsup _{h \rightarrow 0^{+}} \frac{V\left(E_{h}(x ; v)\right)-V(x)}{h} .
$$

Clearly, we have by virtue of (4.4) and (5.5):

$$
\begin{aligned}
V\left(E_{h}(x ; v)\right)= & \max \left\{\max _{\theta \in[-r,-h]} \exp (2 \sigma \theta) Q(x(\theta+h)), \max _{\theta \in[-h, 0]} \exp (2 \sigma \theta) Q(x(0)+(\theta+h) v)\right\} \\
= & \max \left\{\max _{\theta \in[-h, 0]} \exp (2 \sigma \theta)\left[Q(x(0))+(\theta+h) \nabla Q(x(0)) v+\int_{0}^{\theta+h}(\nabla Q(x(0)+s v)-\nabla Q(x(0))) v \mathrm{~d} s\right]\right\} \\
\leqslant & \max \left\{\exp (-2 \sigma h) V(x), \max _{\theta \in[-h, 0]} \exp (2 \sigma \theta)[Q(x(0))+(\theta+h) \nabla Q(x(0)) v]\right. \\
& \left.+h|v| \max _{s \in[0, h]}|\nabla Q(x(0)+s v)-\nabla Q(x(0))|\right\} .
\end{aligned}
$$


If $Q(x(0))<V(x)$ then there exists $h>0$ such that $Q(x(0))+s \nabla Q(x(0)) v \leqslant \frac{1}{2}(V(x)+Q(x(0)))$ for all $s \in[0, h]$. Consequently, in this case we have from (A10) for $h>0$ sufficiently small:

$$
\begin{aligned}
& h^{-1}\left(V\left(E_{h}(x ; v)\right)-V(x)\right) \leqslant \\
& \quad \max \left\{\frac{\exp (-2 \sigma h)-1}{h} V(x), \exp (-2 \sigma h) \frac{1}{2 h}(Q(x(0))-V(x))+|v| \max _{s \in[0, h]}|\nabla Q(x(0)+s v)-\nabla Q(x(0))|\right\} .
\end{aligned}
$$

The above inequality gives (5.6a) for the case $Q(x(0))<V(x)$.

If $Q(x(0))=V(x)$ and $\nabla Q(x(0)) v>-2 \sigma V(x)$ then it follows that $\max _{\theta \in[-h, 0]} \exp (2 \sigma \theta)[Q(x(0))+$ $(\theta+h) \nabla Q(x(0)) v]=Q(x(0))+h \nabla Q(x(0)) v$ for $h>0$ sufficiently small. Consequently, we obtain from (A10):

$h^{-1}\left(V\left(E_{h}(x ; v)\right)-V(x)\right) \leqslant \max \left\{\frac{\exp (-2 \sigma h)-1}{h} V(x), \nabla Q(x(0)) v+|v| \max _{s \in[0, h]}|\nabla Q(x(0)+s v)-\nabla Q(x(0))|\right\}$.

The above inequality gives (5.6b) for the case $Q(x(0))=V(x)$ and $\nabla Q(x(0)) v>-2 \sigma V(x)$.

If $Q(x(0))=V(x)$ and $\nabla Q(x(0)) v \leqslant-2 \sigma V(x)$ then it follows that $\max _{\theta \in[-h, 0]} \exp (2 \sigma \theta)[Q(x(0))+(\theta+h) \times$ $\nabla Q(x(0)) v]=\exp (-2 \sigma h) Q(x(0))$ for $h>0$ sufficiently small. Consequently, we obtain from (A10):

$$
h^{-1}\left(V\left(E_{h}(x ; v)\right)-V(x)\right) \leqslant \frac{\exp (-2 \sigma h)-1}{h} V(x)+|v| \max _{s \in[0, h]}|\nabla Q(x(0)+s v)-\nabla Q(x(0))|
$$

The above inequality gives (5.6b) for the case $Q(x(0))=V(x)$ and $\nabla Q(x(0)) v \leqslant-2 \sigma V(x)$.

Thus inequality (5.6b) holds for the case $Q(x(0))=V(x)$. The proof is complete.

Proof of Claim made in the proof of Theorem 5.1. The proof is made by induction. It is straightforward to verify that definitions (5.8), (5.9a) and (5.9b) guarantee that (5.15) holds for $i=1$. We next assume that inequality (5.15) holds for $i-1(i \geqslant 2)$, i.e.,

$$
\begin{aligned}
& \sum_{j=1}^{i-1}\left(\xi_{j}-k_{j-1}\left(\xi_{1}, \ldots, \xi_{j-1}\right)\right)^{2} b_{j}\left(s^{\prime}\right) \mu_{j}\left(\xi_{1}, \ldots, \xi_{j}\right) \\
& \quad+s^{\prime} \sum_{j=1}^{i-1}\left|\xi_{j}-k_{j-1}\left(\xi_{1}, \ldots, \xi_{j-1}\right)\right| \gamma_{j}\left(s^{\prime}\right)+s^{\prime} \sum_{j=1}^{i-1}\left|\xi_{j}-k_{j-1}\left(\xi_{1}, \ldots, \xi_{j-1}\right)\right| c_{j-1}\left(s^{\prime}\right) \delta_{j-1}\left(\xi_{1}, \ldots, \xi_{j-1}\right) \\
& \leqslant-(n+2-i) \sigma\left(\sum_{j=1}^{i-1}\left|\xi_{j}-k_{j-1}\left(\xi_{1}, \ldots, \xi_{j-1}\right)\right|^{2}\right)
\end{aligned}
$$

where

$$
s^{\prime}=\sum_{j=1}^{i-1}\left|\xi_{j}-k_{j-1}\left(\xi_{1}, \ldots, \xi_{j-1}\right)\right|, \quad c_{0} \equiv 0, \quad c_{j}(s):=\sum_{k=1}^{j} \gamma_{k}(s) \text { for } j=1, \ldots, i-1
$$


By virtue of inequalities (5.12), (A11) and definitions (5.16), (A12), we obtain for all $\left(\xi_{1}, \ldots, \xi_{i}\right)^{\prime} \in \Re^{i}$ :

$$
\begin{gathered}
-\sum_{j=1}^{i}\left(\xi_{j}-k_{j-1}\left(\xi_{1}, \ldots, \xi_{j-1}\right)\right)^{2} b_{j}(s) \mu_{j}\left(\xi_{1}, \ldots, \xi_{j}\right) \\
+s \sum_{j=1}^{i}\left|\xi_{j}-k_{j-1}\left(\xi_{1}, \ldots, \xi_{j-1}\right)\right| \gamma_{j}(s)+s \sum_{j=1}^{i}\left|\xi_{j}-k_{j-1}\left(\xi_{1}, \ldots, \xi_{j-1}\right)\right| c_{j-1}(s) \delta_{j-1}\left(\xi_{1}, \ldots, \xi_{j-1}\right) \\
\leqslant-(n+2-i) \sigma\left(\sum_{j=1}^{i-1}\left|\xi_{j}-k_{j-1}\left(\xi_{1}, \ldots, \xi_{j-1}\right)\right|^{2}\right) \\
-\left(\xi_{i}-k_{i-1}\left(\xi_{1}, \ldots, \xi_{i-1}\right)\right)^{2}\left(b_{i}(s) \mu_{i}\left(\xi_{1}, \ldots, \xi_{i}\right)-\gamma_{i}(s)-c_{i-1}(s) \delta_{i-1}\left(\xi_{1}, \ldots, \xi_{i-1}\right)\right) \\
\quad+\left|\xi_{i}-k_{i-1}\left(\xi_{1}, \ldots, \xi_{i-1}\right)\right| a\left(s, \xi_{1}, \ldots, \xi_{i-1}\right) \sum_{j=1}^{i-1}\left|\xi_{j}-k_{j-1}\left(\xi_{1}, \ldots, \xi_{j-1}\right)\right|
\end{gathered}
$$

where $a\left(s, \xi_{1}, \ldots, \xi_{i-1}\right)$ is defined by (5.14c). Using (A13) in conjunction with the inequality

$$
\begin{aligned}
\left|\xi_{i}-k_{i-1}\left(\xi_{1}, \ldots, \xi_{i-1}\right)\right| \sum_{j=1}^{i-1} \mid \xi_{j}- & k_{j-1}\left(\xi_{1}, \ldots, \xi_{j-1}\right) \mid a_{j}\left(s, \xi_{1}, \ldots, \xi_{i-1}\right) \\
& \leqslant \sigma \sum_{j=1}^{i-1}\left|\xi_{j}-k_{j-1}\left(\xi_{1}, \ldots, \xi_{j-1}\right)\right|^{2}+\left|\xi_{i}-k_{i-1}\left(\xi_{1}, \ldots, \xi_{i-1}\right)\right|^{2} \frac{i-1}{4 \sigma} a^{2}\left(s, \xi_{1}, \ldots, \xi_{i-1}\right)
\end{aligned}
$$

and the fact that the maps $s \rightarrow a\left(s, \xi_{1}, \ldots, \xi_{i-1}\right), s \rightarrow b_{i}(s)$ are non-decreasing and non-increasing, respectively on $\Re^{+}$, the reader is in a position to verify that definition (5.14a) guarantees inequality (5.15). Notice that the inequality $s=\sum_{j=1}^{i}\left|\xi_{j}-k_{j-1}\left(\xi_{1}, \ldots, \xi_{j-1}\right)\right| \leqslant p=\frac{i}{2}+\frac{1}{2} \sum_{j=1}^{i}\left|\xi_{j}-k_{j-1}\left(\xi_{1}, \ldots, \xi_{j-1}\right)\right|^{2}$ is also used. The proof is complete.

Proof of inequalities (5.20) and (5.21). Notice that by virtue of definitions (5.10a), (5.10b) for every $i \geqslant 1$ and $\left(\xi_{1}, \ldots, \xi_{i}\right)^{\prime} \in \Re^{i}$ it holds that

$$
\sum_{j=1}^{i}\left|\xi_{j}\right| \leqslant \sum_{j=1}^{i}\left|\xi_{j}-k_{j-1}\left(\xi_{1}, \ldots, \xi_{j-1}\right)\right|+\sum_{j=1}^{i} \mu_{j-1}\left(\xi_{1}, \ldots, \xi_{j-1}\right)\left|\xi_{j-1}-k_{j-2}\left(\xi_{1}, \ldots, \xi_{j-2}\right)\right| .
$$

Inequality (A14) implies:

$$
\sum_{j=1}^{i}\left|\xi_{j}\right| \leqslant\left(\sum_{j=1}^{i-1}\left(1+\mu_{j}\left(\xi_{1}, \ldots, \xi_{j-1}\right)\right)\right) \max _{j=1, \ldots, i}\left|\xi_{j}-k_{j-1}\left(\xi_{1}, \ldots, \xi_{j-1}\right)\right| .
$$

Definition (5.4) implies that that for all $x \in C^{0}\left([-r, 0] ; \Re^{n}\right)$ with $V(x)=\sum_{j=1}^{n}\left|x_{j}(0)-k_{j-1}\left(x_{1}(0), \ldots, x_{j-1}(0)\right)\right|^{2}$ we have:

$$
\max _{\substack{j=1, \ldots, i \\ \theta \in[-r, 0]}}\left|x_{j}(\theta)-k_{j-1}\left(x_{1}(\theta), \ldots, x_{j-1}(\theta)\right)\right| \leqslant \exp (\sigma r) \sum_{j=1}^{n}\left|x_{j}(0)-k_{j-1}\left(x_{1}(0), \ldots, x_{j-1}(0)\right)\right| .
$$


Inequality (5.21) is a direct consequence of inequalities (5.11c), (A15) and (A16). Consequently, inequalities (5.1) and (5.7) in conjunction with (5.21) imply for all $i \geqslant 1,(t, x, d) \in \Re^{+} \times C^{0}\left([-r, 0] ; \Re^{n}\right) \times D$ with $V(x)=$ $\sum_{j=1}^{n}\left|x_{j}(0)-k_{j-1}\left(x_{1}(0), \ldots, x_{j-1}(0)\right)\right|^{2}:$

$$
\begin{aligned}
\left|f_{i}\left(t, d, x_{1}, \ldots, x_{i}\right)\right| & \leqslant L\left(s \exp (\sigma r) B_{i}(s \exp (\sigma r))\right) B_{i}(s \exp (\sigma r)) s \exp (\sigma r) \\
\frac{1}{\varphi\left(s \exp (\sigma r) B_{i}(s \exp (\sigma r))\right)} & \leqslant g_{i}\left(t, d, x_{1}, \ldots, x_{i}\right) \leqslant \varphi\left(s \exp (\sigma r) B_{i}(s \exp (\sigma r))\right)
\end{aligned}
$$

where

$$
s:=\sum_{j=1}^{n}\left|x_{j}(0)-k_{j-1}\left(x_{1}(0), \ldots, x_{j-1}(0)\right)\right| .
$$

Using (A17) and (A18), we obtain by virtue of definitions (5.11a), (5.11b):

$$
\begin{gathered}
\left|f_{i}\left(t, d, x_{1}, \ldots, x_{i}\right)\right|+\left|g_{i}\left(t, d, x_{1}, \ldots, x_{i}\right)\right|\left|x_{i+1}(0)-k_{i}\left(x_{1}(0), \ldots, x_{i}(0)\right)\right| \leqslant s \gamma_{i}(s), \quad i=1, \ldots, n-1 \\
\left|f_{n}\left(t, d, x_{1}, \ldots, x_{n}\right)\right| \leqslant s \gamma_{n}(s) \\
b_{i}(s) \leqslant g_{i}\left(t, d, x_{1}, \ldots, x_{i}\right) \leqslant \gamma_{i}(s), \quad i=1, \ldots, n
\end{gathered}
$$

where $s$ is defined by (A19). Definition (5.19) in conjunction with definitions (5.10a), (5.10b) gives for $u=k_{n}\left(x_{1}(0), \ldots, x_{n}(0)\right)$ :

$$
\begin{aligned}
A(t, d, x, u) \leqslant & -\sum_{j=1}^{n} g_{j}\left(t, d, x_{1}, \ldots, x_{j}\right) \mu_{j}\left(x_{1}(0), \ldots, x_{j}(0)\right)\left(x_{j}(0)-k_{j-1}\left(x_{1}(0), \ldots, x_{j-1}(0)\right)\right)^{2} \\
& +\sum_{j=1}^{n-1}\left|x_{j}(0)-k_{j-1}\left(x_{1}(0), \ldots, x_{j-1}(0)\right)\right| \mid f_{j}\left(t, d, x_{1}, \ldots, x_{j}\right) \\
& +g_{j}\left(t, d, x_{1}, \ldots, x_{j}\right)\left(x_{j+1}(0)-k_{j}\left(x_{1}(0), \ldots, x_{j}(0)\right)\right) \mid \\
& +\sum_{j=2}^{n-1}\left|\nabla k_{j-1}\left(x_{1}(0), \ldots, x_{j-1}(0)\right)\right|\left|x_{j}(0)-k_{j-1}\left(x_{1}(0), \ldots, x_{j-1}(0)\right)\right| \sum_{l=1}^{j-1} \mid f_{l}\left(t, d, x_{1}, \ldots, x_{l}\right) \\
& +g_{l}\left(t, d, x_{1}, \ldots, x_{l}\right)\left(x_{l+1}(0)-k_{l}\left(x_{1}(0), \ldots, x_{l}(0)\right)\right) \mid \\
& +\sum_{j=2}^{n-1}\left|\nabla k_{j-1}\left(x_{1}(0), \ldots, x_{j-1}(0)\right)\right|\left|x_{j}(0)-k_{j-1}\left(x_{1}(0), \ldots, x_{j-1}(0)\right)\right| \\
& \times \sum_{l=1}^{j-1}\left|x_{l}(0)-k_{l-1}\left(x_{1}(0), \ldots, x_{l-1}(0)\right)\right| \mu_{l}\left(x_{1}(0), \ldots, x_{l}(0)\right)\left|g_{l}\left(t, d, x_{1}, \ldots, x_{l}\right)\right| \\
& +\left|x_{n}(0)-k_{n-1}\left(x_{1}(0), \ldots, x_{n-1}(0)\right)\right|\left|f_{n}(t, d, x)\right| \\
& +\left|\nabla k_{n-1}\left(x_{1}(0), \ldots, x_{n-1}(0)\right)\right|\left|x_{n}(0)-k_{n-1}\left(x_{1}(0), \ldots, x_{n-1}(0)\right)\right| \sum_{l=1}^{n-1} \mid f_{l}\left(t, d, x_{1}, \ldots, x_{l}\right) \\
& +g_{l}\left(t, d, x_{1}, \ldots, x_{l}\right)\left(x_{l+1}(0)-k_{l}\left(x_{1}(0), \ldots, x_{l}(0)\right)\right) \mid \\
& +\left|\nabla k_{n-1}\left(x_{1}(0), \ldots, x_{n-1}(0)\right)\right|\left|x_{n}(0)-k_{n-1}\left(x_{1}(0), \ldots, x_{n-1}(0)\right)\right| \\
& \times \sum_{l=1}^{n-1}\left|x_{l}(0)-k_{l-1}\left(x_{1}(0), \ldots, x_{l-1}(0)\right)\right| \mu_{l}\left(x_{1}(0), \ldots, x_{l}(0)\right)\left|g_{l}\left(t, d, x_{1}, \ldots, x_{l}\right)\right| .
\end{aligned}
$$


Combining (A23) with (A19), (A20), (A21) and (A22) we obtain for $u=k_{n}\left(x_{1}(0), \ldots, x_{n}(0)\right)$ :

$$
\begin{gathered}
A(t, d, x, u) \leqslant-\sum_{j=1}^{n} b_{j}(s) \mu_{j}\left(x_{1}(0), \ldots, x_{j}(0)\right)\left(x_{j}(0)-k_{j-1}\left(x_{1}(0), \ldots, x_{j-1}(0)\right)\right)^{2} \\
+s \sum_{j=2}^{n}\left|x_{j}(0)-k_{j-1}\left(x_{1}(0), \ldots, x_{j-1}(0)\right)\right| \gamma_{j}(s) \\
+s \sum_{j=2}^{n}\left|\nabla k_{j-1}\left(x_{1}(0), \ldots, x_{j-1}(0)\right)\right|\left|x_{j}(0)-k_{j-1}\left(x_{1}(0), \ldots, x_{j-1}(0)\right)\right| \sum_{l=1}^{j-1}\left(1+\mu_{l}\left(x_{1}(0), \ldots, x_{l}(0)\right)\right) \gamma_{l}(s) .
\end{gathered}
$$

Inequality (A24) in conjunction with inequality (5.13) implies for $u=k_{n}\left(x_{1}(0), \ldots, x_{n}(0)\right)$ :

$$
\begin{aligned}
A(t, d, x, u) \leqslant & -\sum_{j=1}^{n} b_{j}(s) \mu_{j}\left(x_{1}(0), \ldots, x_{j}(0)\right)\left(x_{j}(0)-k_{j-1}\left(x_{1}(0), \ldots, x_{j-1}(0)\right)\right)^{2} \\
& +s \sum_{j=1}^{n}\left|x_{j}(0)-k_{j-1}\left(x_{1}(0), \ldots, x_{j-1}(0)\right)\right| \gamma_{j}(s)+s \sum_{j=1}^{n} \delta_{j-1}\left(x_{1}(0), \ldots, x_{j-1}(0)\right) \mid x_{j}(0) \\
& -k_{j-1}\left(x_{1}(0), \ldots, x_{j-1}(0)\right) \mid \sum_{l=1}^{j-1} \gamma_{l}(s) .
\end{aligned}
$$

Clearly, inequality (A25) in conjunction with (5.15) for $i=n$ show that (5.20) holds. The proof is complete.

Acknowledgements. The work of Z.-P. Jiang has been supported in part by NSF grants ECS-0093176 and DMS-0504462.

\section{REFERENCES}

[1] D. Aeyels and J. Peuteman, A new asymptotic stability criterion for nonlinear time-variant differential equations. IEEE Trans. Automat. Contr. 43 (1998) 968-971.

[2] Z. Artstein, Stabilization with relaxed controls. Nonlinear Anal. Theory Methods Appl. 7 (1983) 1163-1173.

[3] J.P. Aubin and H. Frankowska, Set-Valued Analysis. Birkhauser, Boston, USA (1990).

[4] F.H. Clarke, Y.S. Ledyaev, E.D. Sontag and A.I. Subbotin, Asymptotic controllability implies feedback stabilization. IEEE Trans. Automat. Contr. 42 (1997) 1394-1407.

[5] J.-M. Coron, Control and Nonlinearity, Mathematical Surveys and Monographs 136. AMS, USA (2007).

[6] J.-M. Coron and L. Rosier, A relation between continuous time-varying and discontinuous feedback stabilization. J. Math. Syst. Estim. Contr. 4 (1994) 67-84.

[7] R.A. Freeman and P.V. Kokotovic, Robust Nonlinear Control Design-State Space and Lyapunov Techniques. Birkhauser, Boston, USA (1996).

[8] J.K. Hale and S.M.V. Lunel, Introduction to Functional Differential Equations. Springer-Verlag, New York, USA (1993).

[9] C. Hua, G. Feng and X. Guan, Robust controller design of a class of nonlinear time delay systems via backstepping methods. Automatica 44 (2008) 567-573.

[10] M. Jankovic, Control Lyapunov-Razumikhin functions and robust stabilization of time delay systems. IEEE Trans. Automat. Contr. 46 (2001) 1048-1060.

[11] M. Jankovic, Stabilization of Nonlinear Time Delay Systems with Delay Independent Feedback, in Proceedings of the 2005 American Control Conference, Portland, OR, USA (2005) 4253-4258.

[12] Z.-P. Jiang, Y. Lin and Y. Wang, Stabilization of time-varying nonlinear systems: A control Lyapunov function approach, in Proceedings of IEEE International Conference on Control and Automation 2007, Guangzhou, China (2007) 404-409.

[13] I. Karafyllis, The non-uniform in time small-gain theorem for a wide class of control systems with outputs. Eur. J. Contr. 10 (2004) 307-323.

[14] I. Karafyllis, Non-uniform in time robust global asymptotic output stability. Syst. Contr. Lett. 54 (2005) $181-193$.

[15] I. Karafyllis, Lyapunov theorems for systems described by retarded functional differential equations. Nonlinear Anal. Theory Methods Appl. 64 (2006) 590-617. 
[16] I. Karafyllis, A system-theoretic framework for a wide class of systems I: Applications to numerical analysis. J. Math. Anal. Appl. 328 (2007) 876-899.

[17] I. Karafyllis and C. Kravaris, Robust output feedback stabilization and nonlinear observer design. Syst. Contr. Lett. 54 (2005) 925-938.

[18] I. Karafyllis and J. Tsinias, A converse Lyapunov theorem for non-uniform in time global asymptotic stability and its application to feedback stabilization. SIAM J. Contr. Optim. 42 (2003) 936-965.

[19] I. Karafyllis and J. Tsinias, Control Lyapunov functions and stabilization by means of continuous time-varying feedback. ESAIM: COCV 15 (2009) 599-625.

[20] I. Karafyllis, P. Pepe and Z.-P. Jiang, Global output stability for systems described by retarded functional differential equations: Lyapunov characterizations. Eur. J. Contr. 14 (2008) 516-536.

[21] M. Krstic, I. Kanellakopoulos and P.V. Kokotovic, Nonlinear and Adaptive Control Design. John Wiley (1995).

[22] Y. Lin, E.D. Sontag and Y. Wang, A smooth converse Lyapunov theorem for robust stability. SIAM J. Contr. Optim. 34 (1996) 124-160.

[23] F. Mazenc and P.-A. Bliman, Backstepping design for time-delay nonlinear systems. IEEE Trans. Automat. Contr. 51 (2006) $149-154$.

[24] F. Mazenc, M. Malisoff and Z. Lin, On input-to-state stability for nonlinear systems with delayed feedbacks, in Proceedings of the American Control Conference (2007), New York, USA (2007) 4804-4809.

[25] E. Moulay and W. Perruquetti, Stabilization of non-affine systems: A constructive method for polynomial systems. IEEE Trans. Automat. Contr. 50 (2005) 520-526.

[26] S.K. Nguang, Robust stabilization of a class of time-delay nonlinear systems. IEEE Trans. Automat. Contr. 45 (2000) $756-762$.

[27] J. Peuteman and D. Aeyels, Exponential stability of nonlinear time-varying differential equations and partial averaging. Math. Contr. Signals Syst. 15 (2002) 42-70.

[28] J. Peuteman and D. Aeyels, Exponential stability of slowly time-varying nonlinear systems. Math. Contr. Signals Syst. 15 (2002) 202-228.

[29] L. Praly, G. Bastin, J.-B. Pomet and Z.P. Jiang, Adaptive stabilization of nonlinear systems, in Foundations of Adaptive Control, P.V. Kokotovic Ed., Springer-Verlag (1991) 374-433.

[30] E.D. Sontag, A universal construction of Artstein's theorem on nonlinear stabilization. Syst. Contr. Lett. 13 (1989) $117-123$.

[31] E.D. Sontag and Y. Wang, Notions of input to output stability. Syst. Contr. Lett. 38 (1999) $235-248$.

[32] E.D. Sontag, and Y. Wang, Lyapunov characterizations of input-to-output stability. SIAM J. Contr. Optim. 39 (2001) $226-249$.

[33] J. Tsinias, Sufficient Lyapunov-like conditions for stabilization. Math. Contr. Signals Syst. 2 (1989) 343-357.

[34] J. Tsinias and N. Kalouptsidis, Output feedback stabilization. IEEE Trans. Automat. Contr. 35 (1990) 951-954.

[35] S. Zhou, G. Feng and S.K. Nguang, Comments on robust stabilization of a class of time-delay nonlinear systems. IEEE Trans. Automat. Contr. 47 (2002) 1586-1586. 